\title{
Simulating diverse instabilities of dust in magnetized gas
}

\author{
Philip F. Hopkins ${ }^{\circledR},{ }^{\star}{ }^{\star}$ Jonathan Squire $^{\circledR 2}$ and Darryl Seligman ${ }^{3}$ \\ ${ }^{1}$ TAPIR, Mailcode 350-17, California Institute of Technology, Pasadena, CA 91125, USA \\ ${ }^{2}$ Physics Department, University of Otago, 730 Cumberland St, Dunedin 9016, New Zealand \\ ${ }^{3}$ Department of Astronomy, Yale University, 52 Hillhouse Ave., New Haven, CT 06511, USA
}

Accepted 2020 April 16. Received 2020 April 14; in original form 2019 April 26

\begin{abstract}
Recently, Squire \& Hopkins showed that charged dust grains moving through magnetized gas under the influence of a uniform external force (such as radiation pressure or gravity) are subject to a spectrum of instabilities. Qualitatively distinct instability families are associated with different Alfvén or magnetosonic waves and drift or gyro motion. We present a suite of simulations exploring these instabilities, for grains in a homogeneous medium subject to an external acceleration. We vary parameters such as the ratio of Lorentz-to-drag forces on dust, plasma $\beta$, size scale, and acceleration. All regimes studied drive turbulent motions and dust-to-gas fluctuations in the saturated state, rapidly amplify magnetic fields into equipartition with velocity fluctuations, and produce instabilities that persist indefinitely (despite random grain motions). Different parameters produce diverse morphologies and qualitatively different features in dust, but the saturated gas state can be broadly characterized as anisotropic magnetosonic or Alfvénic turbulence. Quasi-linear theory can qualitatively predict the gas turbulent properties. Turbulence grows from small to large scales, and larger scale modes usually drive more vigorous gas turbulence, but dust velocity and density fluctuations are more complicated. In many regimes, dust forms structures (clumps, filaments, sheets) that reach extreme overdensities (up to $\gg 10^{9}$ times mean), and exhibit substantial substructure even in nearly incompressible gas. These can be even more prominent at lower dust-to-gas ratios. In other regimes, dust self-excites scattering via magnetic fluctuations that isotropize and amplify dust velocities, producing fast, diffusive dust motions.
\end{abstract}

Key words: accretion, accretion discs - instabilities - turbulence - planets and satellites: formation-ISM: kinematics and dynamics - galaxies: formation.

\section{INTRODUCTION}

Almost all astrophysical, planetary, and atmospheric fluids are laden with grains of dust, which play a central role in many astrophysical processes including in planet and star formation; in the attenuation and extinction of observed light; in cool star, brown dwarf, and planetary evolution; in atmospheric dynamics; in astrochemistry; in feedback and outflow launching from star-forming regions, cool stars, and active galactic nuclei (AGNs); and in interstellar cooling and heating (see Dorschner 2003; Draine 2003; Apai \& Lauretta 2010, for reviews). The dynamical interactions between dust and gas therefore are of fundamental importance in astrophysics.

Recently, Squire \& Hopkins (2018b) showed that dust-gas mixtures are unstable to a broad class of instabilities, which they referred to as 'resonant drag instabilities' (RDIs). The Squire \&

^E-mail: phopkins@caltech.edu
Hopkins (2018b) instabilities manifest whenever a fluid, gas, or plasma system contains dust streaming with non-zero drift velocity $\mathbf{w}_{\mathrm{s}} \equiv \mathbf{v}_{\mathrm{d}}-\mathbf{u}_{\mathrm{g}}$ relative to the gas (where $\mathbf{v}_{\mathrm{d}}$ and $\mathbf{u}_{\mathrm{g}}$ are the dust and gas velocities, respectively). Although a broad range of wavelengths are unstable, the resonances which produce the most rapidly growing instabilities occur when the natural frequency of a linear gas mode [e.g. a sound wave, magnetohydrodynamic (MHD) wave, or epicyclic oscillation] matches the natural frequency of a dust mode (e.g. advection, with frequency $\mathbf{w}_{\mathrm{s}} \cdot \mathbf{k}$, or gyro oscillations). Every such pair of modes produces an instability, with a unique growth rate, resonance, and linear mode structure. Since dust-gas drift may be caused by many external forces, such as radiative absorption or scattering by dust or gas, gravity in quasi-hydrostatic systems, centrifugal or coriolis forces in rotating systems, or large-scale hydrodynamic or pressure forces, these instabilities will develop in a range of astrophysical environments.

In a series of papers, Hopkins \& Squire (2018a,b) and Squire \& Hopkins (2018a) analytically explored various examples of these instabilities in some astrophysical systems. Hopkins \& Squire (2018a, 
Table 1. The default initial conditions for the simulations studied in this paper. Each simulation (by default) adopts an isothermal gas equation of state, Epstein drag, constant grain charge, and follows a single population of grains, with resolution $2 \times 128^{3}$ elements (equal number gas and dust), and total dust-to-gas mass ratio $\mu \equiv \rho_{\mathrm{d}}^{0} / \rho_{\mathrm{g}}^{0}=0.01$. These choices are varied below. Columns show: (1) Simulation name (used throughout). (2) $\left|\mathbf{w}_{\mathrm{s}}^{0}\right| / c_{\mathrm{s}}^{0}$ : The initial equilibrium drift velocity, in units of the sound speed. (2) $\tau \equiv t_{\mathrm{s}}^{0} / t_{\mathrm{L}}^{0}$ : Ratio of Lorentz to drag forces (stopping time to gyro time). (3) $\left|\hat{\mathbf{B}}_{0} \cdot \hat{\mathbf{a}}\right|:$ Angle between initial magnetic field and direction of differential acceleration/force between dust and gas, $\mathbf{a} \equiv \mathbf{a}_{\mathrm{ext}}$, dust $-\mathbf{a}_{\mathrm{ext}}$ gas. (4) $L_{\mathrm{box}} / c_{\mathrm{s}}^{0} t_{\mathrm{s}}^{0}$ : Box size in dimensionless units. (5) $\beta \equiv P_{\text {gas }}^{0} / P_{B}^{0}=2\left(c_{\mathrm{s}}^{0} / v_{\mathrm{A}}^{0}\right)^{2}$ : Initial ratio of gas thermal to magnetic pressure. (6) Notes: we provide an example physical regime where these parameters are plausible for typical interstellar grains (from Paper I). We also quote an equivalent set of dimensionless parameters: the 'acceleration parameter' $\tilde{a} \equiv|\mathbf{a}| \bar{\rho}_{\text {grain }}^{i} \epsilon_{\text {grain }} /\left(\left(c_{\mathrm{s}}^{0}\right)^{2} \rho_{\mathrm{g}}^{0}\right)$, 'charge parameter' $\tilde{\phi} \equiv 3 Z_{\text {grain }}^{0} e /\left(4 \pi c \epsilon_{\text {grain }}^{2}\left(\rho_{\mathrm{g}}^{0}\right)^{1 / 2}\right)$, and 'size parameter' $\tilde{\alpha} \equiv \bar{\rho}_{\text {grain }}^{i} \epsilon_{\text {grain }} / \rho_{\mathrm{g}}^{0} L_{\text {box }}$.

\begin{tabular}{|c|c|c|c|c|c|c|}
\hline Name & $\frac{\left|\mathbf{w}_{\mathrm{s}}^{0}\right|}{c_{\mathrm{s}}^{0}}(\tilde{a})$ & $\tau(\tilde{\phi})$ & $\left|\hat{\mathbf{B}}_{0} \cdot \hat{\mathbf{a}}\right|$ & $\frac{L_{\mathrm{box}}}{c_{\mathrm{s}}^{0} t_{\mathrm{s}}^{0}}(\tilde{\alpha})$ & $\beta$ & Notes \\
\hline Example & $0.89(25)$ & $29(50)$ & 0.05 & $0.34(5)$ & 2 & Case study in Seligman, Hopkins \& Squire (2019) \\
\hline $\begin{array}{l}\text { AGB } \\
(\mathrm{S} / \mathrm{M} / \mathrm{L} / \mathrm{XL})\end{array}$ & $3(8.4)$ & $1 e-3(2.8 \mathrm{e}-3)$ & $1 / \sqrt{2}$ & $\begin{array}{c}0.01,3.1,920,3 \mathrm{e} 5 \\
(270,0.9,0.003,1 \mathrm{e}-5)\end{array}$ & 2 & $\begin{array}{l}\text { AGB wind, } r \sim 100 R_{\odot}, \dot{M} \sim 10^{-6} M_{\odot} \mathrm{yr}^{-1} \\
L_{\text {box }} \sim\left(5 \mathrm{~km}, 2000 \mathrm{~km}, 1 R_{\odot}, 300 R_{\odot}\right)\end{array}$ \\
\hline $\begin{array}{l}\text { H II-near } \\
(\mathrm{S} / \mathrm{M} / \mathrm{L})\end{array}$ & $4(20)$ & $2.3(24)$ & $1 / \sqrt{2}$ & $\begin{array}{l}0.0088,2.6,1000 \\
(390,1.3,0.0034)\end{array}$ & 20 & $\begin{array}{l}\text { H II region, } r=0.1 \mathrm{pc}, n=10^{4} \mathrm{~cm}^{-3} \\
L_{\text {box }} \sim(0.2 \mathrm{au}, 50 \mathrm{au}, 0.1 \mathrm{pc})\end{array}$ \\
\hline $\begin{array}{l}\text { WIM } \\
(\mathrm{S} / \mathrm{M} / \mathrm{L})\end{array}$ & $0.05(0.12)$ & $100(160)$ & $1 / \sqrt{2}$ & $\begin{array}{l}4.8 \mathrm{e}-4,0.21,100 \\
(3400,7.6,0.017)\end{array}$ & 2 & $\begin{array}{l}\text { WIM, } n \sim 1 \mathrm{~cm}^{-3}, T \sim 10^{4} \mathrm{~K} \\
L_{\text {box }} \sim(1 \mathrm{au}, 500 \mathrm{au}, 1 \mathrm{pc})\end{array}$ \\
\hline $\begin{array}{l}\text { Corona } \\
(\mathrm{S} / \mathrm{M} / \mathrm{L})\end{array}$ & $20(480)$ & $3200(1700)$ & $1 / \sqrt{2}$ & $\begin{array}{l}3.3 \mathrm{e}-4,0.067,20 \\
(4.8 \mathrm{e} 4,240,0.8)\end{array}$ & 0.002 & $\begin{array}{l}\text { Solar corona, } r \sim R_{\odot} \\
L_{\text {box }} \sim\left(10 \mathrm{~km}, 2000 \mathrm{~km}, 1 R_{\odot}\right)\end{array}$ \\
\hline CGM & $9.5(100)$ & $1.4 \mathrm{e} 5(3 \mathrm{e} 7)$ & $1 / \sqrt{2}$ & $0.29(25)$ & 2000 & CGM at $100 \mathrm{kpc}$ from $\mathrm{QSO} ; L_{\mathrm{box}} \sim 1 \mathrm{kpc}$ \\
\hline
\end{tabular}

hereafter Paper I) focused on the case of instabilities involving charged dust in magnetized gas, relevant in the warm interstellar medium (ISM), circum and intergalactic medium (CGM/IGM), $\mathrm{H}$ II regions, supernovae ( $\mathrm{SNe}$ ) ejecta and remnants, the Solar and stellar coronae, cool star winds, AGN outflows and obscuring torii, and giant molecular clouds (GMCs). They showed that a variety of instabilities appear with different properties and growth rate scalings, even in the case of a homogeneous gas obeying ideal MHD (a good approximation in most of these regimes), with a single group of grains interacting via drag and Lorentz forces. However, their analysis was restricted to analytic, linear perturbation theory. Moseley, Squire \& Hopkins (2019) presented simulations of the un-magnetized and un-charged instabilities in the non-linear regime. Seligman et al. (2019) presented a case study of one example in the magnetized regime, and found that the introduction of a magnetic field produced novel dust behaviours and outcomes in both the linear and non-linear regimes of the instability. That first study necessarily neglected much of the large parameter space.

In this paper, we present a large survey of $\sim 40$ simulations $^{1}$ that explore the non-linear regime of these instabilities in a representative range of the astrophysically relevant parameter space for charged dust in magnetized gas. These idealized experiments inform our understanding of the mechanisms responsible for the growth and saturation of the instabilities, the non-linear structure of the dust and gas, and the potential theoretical and observational ramifications. They are complex because the instabilities depend on six dimensionless parameters, and as shown in Paper I, at any given wavenumber $\mathbf{k}$, the linear dispersion relation typically features $\sim 3$ 7 unstable modes (each of which has growth rates that depend on the mode angle $\hat{\mathbf{k}}$, at a given $|\mathbf{k}|$ ). This inherent complexity further

\footnotetext{
${ }^{1}$ Animations and additional visualizations of the simulations here are available at http://www.tapir.caltech.edu $/ \sim$ phopkins/Site/animations/dust-a nd-gas-in-astrophysic.
}

underscores the necessity for numerical simulations that explore different non-linear regimes. We show that a diverse variety of behaviours arise, depending both on the physical parameters of the system and the spatial scales studied, all of which may have important astrophysical consequences.

This paper is organized as follows. Section 2 presents our methodology, and Section 3 discusses the parameter space surveyed (see also Table 1 and Fig. 1). Section 4 presents several results from the simulations [e.g. morphologies, saturated fluctuation amplitudes, and probability distribution functions (PDFs)]. Section 5 discusses these results in more detail and compares them to theoretical expectations, attempting to identify classes of saturation mechanisms. We summarize and conclude in Section 7.

\section{METHODS AND SIMULATION SET-UP}

\subsection{Numerical methods and equations solved}

The numerical methods adopted here have been described in detail in Moseley et al. (2019) and Seligman et al. (2019), and we briefly summarize them here. Our simulations were run with the code GIZMO (Hopkins 2015), ${ }^{2}$ using the Lagrangian 'meshless finite volume' (MFV) method for MHD, which has been extensively tested on problems involving multifluid MHD instabilities, MRI, shock capturing, and more (Hopkins 2016b, 2017; Hopkins \& Raives 2016; Su et al. 2017). Grains are integrated using the 'superparticle' method (see e.g. Carballido, Stone \& Turner 2008; Johansen, Youdin \& Mac Low 2009; Bai \& Stone 2010b; Pan et al. 2011), whereby the motion of each dust 'particle' in the simulation follows equation (1) below, but each represents an ensemble of dust grains with similar size, mass, and charge (denoted $\epsilon_{\text {grain }}, m_{\text {grain }}$, $q_{\text {grain }}$, respectively). The numerical methods used for the integration

\footnotetext{
${ }^{2} \mathrm{~A}$ public version of the code, including all methods used in this paper, is
} available at http://www.tapir.caltech.edu/ phopkins/Site/GIZMO.html. 


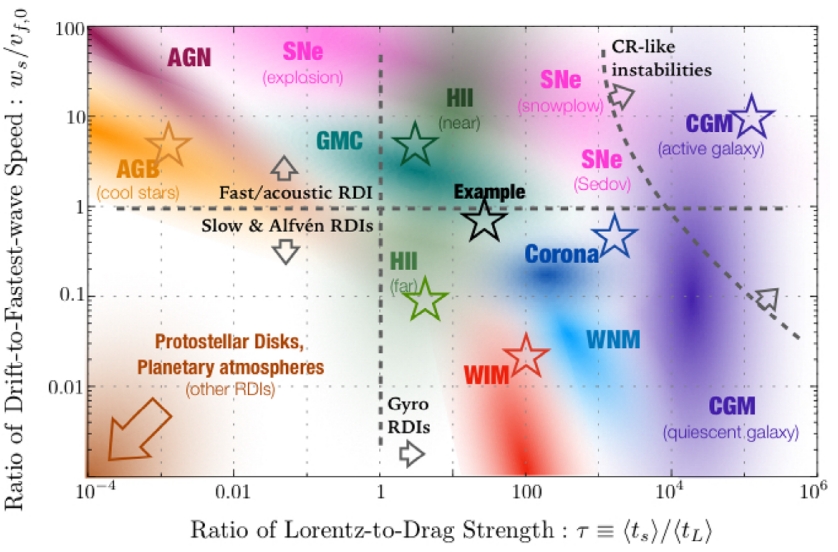

Figure 1. The simulations studied in this work are shown with stars (see Table 1), in an illustration of two important parameters of the MHD RDI (adapted from Paper I). Axes show dust drift speed normalized by the fastest wave speed $\left(w_{\mathrm{s}} / v_{\mathrm{f}, 0} \equiv\left|\mathbf{w}_{\mathrm{s}}\right| /\left(c_{\mathrm{s}}^{2}+v_{\mathrm{A}}^{2}\right)^{1 / 2}\right)$, and the ratio of Lorentz force to drag force (or drag/stopping time to gyro time, $\tau \equiv\left\langle t_{\mathrm{S}}\right\rangle /\left\langle t_{\mathrm{L}}\right\rangle$ ). Different shaded regions illustrate different astrophysical environments (see Paper I, section 9), including the warm ionized and warm neutral medium (WIM/WNM), giant molecular clouds (GMCs) and near/far vicinity of $\mathrm{O}$ stars in $\mathrm{H}$ II regions (H II), supernovae in various phases of evolution (SNe), stellar coronal dust (Corona), cool/giant/AGB star photospheres and outflows (AGB), dusty 'torii' around AGNs, the circum and/or intergalactic medium around AGN/starburst systems or quiescent galaxies (CGM), and protostellar/planetary discs and planetary atmospheres (which extend off the plotted range). Lines/arrows illustrate where different forms of the RDIs should appear: the fast (acoustic) RDI is unstable for $w_{\mathrm{s}} / v_{\mathrm{f}, 0} \gtrsim 1$, gyroresonant RDIs can be dominant at $\tau \gtrsim 1$, and cosmic ray-like RDIs can dominate at very large $\tau$.

are described and tested in Hopkins \& Lee (2016) and Lee, Hopkins $\&$ Squire (2017). The backreaction is accounted for as in Moseley et al. (2019, see appendix B) and the Lorentz forces is evolved using a Boris integrator.

Each individual grain (dust superparticle) in the code obeys

$\frac{\mathrm{d} \mathbf{v}_{\mathrm{d}}}{\mathrm{d} t}=-\frac{\mathbf{w}_{\mathrm{s}}}{t_{\mathrm{s}}}-\frac{\mathbf{w}_{\mathrm{s}} \times \hat{\mathbf{B}}}{t_{\mathrm{L}}}+\mathbf{a}_{\mathrm{ext}, \text { dust }}$,

where $\mathbf{a}_{\text {ext, dust }}$ is a constant external acceleration, $t_{\mathrm{s}}$ is the drag coefficient or 'stopping time', and $t_{\mathrm{L}}$ the gyro or Larmor time. The gas obeys the ideal MHD equations - the standard advection equation, $\partial \rho_{\mathrm{g}} / \partial t=-\nabla \cdot\left(\rho_{\mathrm{g}} \mathbf{u}_{\mathrm{g}}\right)$, for the gas density $\rho_{\mathrm{g}}$, and the standard induction equation, $\partial \mathbf{B} / \partial t=\nabla \times\left(\mathbf{u}_{\mathrm{g}} \times \mathbf{B}\right)$, for the magnetic field $\mathbf{B}$ - with the addition of a backreaction force from the grains in the momentum equation. In particular, whenever drag or Lorentz forces exert a force $m_{\text {grain }} \mathrm{d} \mathbf{v}_{\mathrm{d}} / \mathrm{d} t$ on a grain within a given gas cell, an equal-but-opposite force is applied to the gas. This is treated as a usual momentum flux within GIZMO, which numerically guarantees exact force balance and total momentum conservation. The gas momentum equation reads

$$
\begin{aligned}
\rho_{\mathrm{g}}\left(\frac{\partial}{\partial t}+\mathbf{u}_{\mathrm{g}} \cdot \nabla\right) \mathbf{u}_{\mathrm{g}}= & -\nabla P-\frac{\mathbf{B} \times(\nabla \times \mathbf{B})}{4 \pi}+\rho_{\mathrm{g}} \mathbf{a}_{\text {ext, gas }} \\
& -\int \mathrm{d}^{3} \mathbf{v}_{\mathrm{d}} f_{\mathrm{d}}\left(\mathbf{x}, \mathbf{v}_{\mathrm{d}}\right) \mathbf{a}_{\text {gas-dust }}\left(\mathbf{v}_{\mathrm{d}}, \ldots\right),
\end{aligned}
$$

where $f_{\mathrm{d}}\left(\mathbf{x}, \mathbf{v}_{\mathrm{d}}\right)$ is the phase-space density distribution of dust (i.e. differential mass of grains per element $\mathrm{d}^{3} \mathbf{x} \mathrm{d}^{3} \mathbf{v}_{\mathrm{d}}$ ) and $\mathbf{a}_{\text {ext, gas }}$ is an external gas acceleration that we set to zero in the simulations here. The gas obeys an exactly polytropic equation of state with thermal pressure $P$ and sound speed $c_{\mathrm{s}}$ :

$$
\begin{aligned}
P & =P_{0}\left(\frac{\rho_{\mathrm{g}}}{\rho_{\mathrm{g}}^{0}}\right)^{\gamma}, \\
c_{\mathrm{s}}^{2} & \equiv \frac{\partial P}{\partial \rho_{\mathrm{g}}} .
\end{aligned}
$$

In our default simulations we assume Epstein drag, which can be approximated to very high accuracy with the expression (valid for both sub and supersonic drift)

$t_{\mathrm{s}} \equiv \sqrt{\frac{\pi \gamma}{8}} \frac{\bar{\rho}_{\text {grain }}^{i} \epsilon_{\text {grain }}}{\rho_{\mathrm{g}} c_{\mathrm{s}}}\left(1+\frac{9 \pi \gamma}{128} \frac{\left|\mathbf{w}_{\mathrm{s}}\right|^{2}}{c_{\mathrm{s}}^{2}}\right)^{-1 / 2}$,

where $\bar{\rho}_{\text {grain }}^{i}$ and $\epsilon_{\text {grain }}$ are the internal grain density and radius, respectively. The Larmor time is

$t_{\mathrm{L}} \equiv \frac{m_{\text {grain }} c}{\left|q_{\text {grain }} \mathbf{B}\right|}=\frac{4 \pi \bar{\rho}_{\text {grain }}^{i} \epsilon_{\text {grain }}^{3} c}{3 e\left|Z_{\text {grain }} \mathbf{B}\right|}$,

where $m_{\text {grain }}$ and $q_{\text {grain }}=Z_{\text {grain }} e$ are the grain mass and charge, respectively.

\subsection{Initial conditions}

We initialize a periodic, cubic box of side length $L_{\mathrm{box}}$ with uniform gas density $\rho_{\mathrm{g}}^{0} \equiv M_{\mathrm{gas}}$ box $/ L_{\text {box }}^{3}$ and dust density $\rho_{\mathrm{d}}^{0} \equiv \mu \rho_{\mathrm{g}}^{0}$ (dustto-gas ratio $\mu)$, gas velocity $\mathbf{u}_{\mathrm{g}}^{0}=0$, and dust drift $\mathbf{w}_{\mathrm{s}}^{0}=|\mathbf{a}| t_{\mathrm{s}}^{0}(1+$ $\mu)^{-1}\left(1+\tau^{2}\right)^{-1}\left[\hat{\mathbf{a}}-\tau\left(\hat{\mathbf{a}} \times \hat{\mathbf{B}}_{0}\right)+\tau^{2}\left(\hat{\mathbf{a}} \cdot \hat{\mathbf{B}}_{0}\right) \hat{\mathbf{B}}_{0}\right]$ (see Paper I, section 3.1). Here,

$X_{0} \equiv\left\langle X\left(\rho_{\mathrm{g}}^{0}, \mathbf{w}_{\mathrm{s}}^{0}, \ldots, t=0\right)\right\rangle$

is the initial homogeneous value of some variable $X, \mathbf{a} \equiv \mathbf{a}_{\mathrm{ext}}$, dust $\mathbf{a}_{\text {ext, gas }}$ is the difference between dust and gas accelerations, and $\tau \equiv t_{\mathrm{S}}^{0} / t_{\mathrm{L}}^{0}$ parametrizes the grains' magnetization. The homogenous, steady-state equilibrium solution preserves this quasiequilibrium while the whole box uniformly accelerates ${ }^{3}$ with $\mathbf{u}_{\mathrm{g}}(\mathbf{x}, t)=\left\langle\mathbf{u}_{\mathrm{g}}(t)\right\rangle=\mathbf{a}_{\text {ext, gas }} t+\mathbf{a} \mu t /(1+\mu)$.

We can make the coupled dust-gas equations dimensionless by working in units of the equilibrium sound speed $c_{\mathrm{s}}^{0}$, gas density $\rho_{\mathrm{g}}^{0}$, and 'weighted grain size' $\bar{\rho}_{\text {grain }}^{i} \epsilon_{\text {grain }} / \rho_{\mathrm{g}}^{0}$. Then, for a given equation of state, the dynamics of the problem (at infinite numerical resolution) are entirely determined by six dimensionless parameters: (1) the acceleration $\tilde{a} \equiv|\mathbf{a}| \bar{\rho}_{\text {grain }}^{i} \epsilon_{\text {grain }} /\left(\left(c_{\mathrm{s}}^{0}\right)^{2} \rho_{\mathrm{g}}^{0}\right),(2)$ the box size or grain 'size parameter' $\tilde{\alpha} \equiv \bar{\rho}_{\text {grain }}^{i} \epsilon_{\text {grain }} / \rho_{\mathrm{g}}^{0} L_{\mathrm{box}},(3)$ the grain 'charge parameter' $\tilde{\phi} \equiv 3 Z_{\text {grain }}^{0} e /\left(4 \pi c \epsilon_{\text {grain }}^{2}\left(\rho_{\mathrm{g}}^{0}\right)^{1 / 2}\right)$, (4) the dust-to-gas ratio $\mu \equiv \rho_{\mathrm{d}}^{0} / \rho_{\mathrm{g}}^{0}$, (5) the plasma $\beta \equiv P_{0} /\left(\left|\mathbf{B}_{0}\right|^{2} / 8 \pi\right)$, and (6) the angle $\left|\cos \theta_{\mathbf{B a}}\right| \equiv\left|\hat{\mathbf{B}}_{0} \cdot \hat{\mathbf{a}}\right|$ between the initial field direction $\hat{\mathbf{B}}_{0}$ and â. Note that in our linear theory perturbation analysis we chose to work with a different, but mathematically equivalent, set of

\footnotetext{
${ }^{3}$ As shown explicitly in Hopkins \& Squire (2018b, see section 2.1 and appendix B therein) and Paper I, the dynamics of this local (un-stratified) problem are manifestly identical in the stationary and free-falling or uniformly accelerating frames moving with the homogeneous solution, or (by extension) if we add an equal-and-opposite mean acceleration on the gas such that the mean acceleration of the entire system vanishes. The problem is trivially invariant to any uniform velocity boost. We therefore will perform all our analysis in the co-moving (free-falling) frame. We have also verified (for numerical testing) that our results are identical up to machine error (as they should be given our Lagrangian code) if we instead add an explicit uniform acceleration and/or boost to gas and dust to ensure the homogenous $\mathbf{u}_{\mathrm{g}}(t)=\mathbf{0}$ in the lab frame.
} 
dimensionless variables: (1) $\left|\mathbf{w}_{\mathrm{s}}^{0}\right| / c_{\mathrm{s}}^{0}$, (2) $L_{\mathrm{box}} / c_{\mathrm{s}}^{0} t_{\mathrm{s}}^{0}$, (3) $\tau$, (4) $\mu$, (5) $\beta$, and (6) $\theta_{\mathbf{B a}}$.

Our default simulations adopt $N_{\text {gas }}=128^{3}$ gas resolution elements and an equal number of dust elements, $\mu=0.01$ (the ISM mean), and an isothermal $(\gamma=1)$ equation of state (appropriate for most ISM/CGM/H II region conditions of interest). But we vary all of this below. For simplicity, we assume throughout that grains are all of the same size and charge, and that the grain charge $q_{\text {grain }}$ is fixed during the simulation (as appropriate for large grains in isothermal gas) with grain Larmor time $t_{\mathrm{L}} \equiv m_{\text {grain }} c /\left|q_{\text {grain }} \mathbf{B}\right|$. The 'non-default' simulations discussed in Section 4.2 relax some of these restrictions, exploring different equations of states, dust-togas ratios, resolutions, and allowing $q_{\text {grain }}$ to vary with local gas parameters.

For convenience, throughout we adopt the Cartesian $(x y z)$ axis convention with $\hat{\mathbf{z}} \propto \mathbf{B}_{0}, \hat{\mathbf{x}} \propto \mathbf{a}_{\perp}$ (i.e. the $x-z$ plane is defined to contain a, so $\mathbf{a}=\mathbf{a}_{\perp}+\mathbf{a}_{\|}=\mathrm{a}_{\perp} \hat{\mathbf{x}}+\mathrm{a}_{\|} \hat{\mathbf{z}}$ ), and $\hat{\mathbf{y}} \propto \mathbf{B}_{0} \times \mathbf{a}$ (the mutually perpendicular direction). In our $3 \mathrm{D}$ visualizations, the width/depth/height dimensions correspond to $x / y / z$.

\section{PARAMETER SPACE EXPLORED}

Because the possible parameter space is enormous (the six dimensions above, plus the choice of equation of state, drag law, and charge law), we do not attempt to survey it systematically, but instead choose several unique parameter combinations motivated by values one might expect in different astrophysical systems. For more information, see section 9 of Paper I, which discusses each of these physical regimes extensively (Fig. 1 is adapted from this). The baseline parameters for our 'default' simulation set are given in Table 1 and illustrated in Fig. 1. We give a brief description of each system in the following paragraphs.

The parameters HII-near and HII-far (from fig. 7 in Paper I) correspond to plausible parameters in a massive $\mathrm{H}$ II region at two different radii from the star(s). Specifically, H II-near corresponds to parameters expected for $\mathrm{H}$ II regions with $\sim 0.1 \mu \mathrm{m}$ grains at a distance $r \sim 0.1 \mathrm{pc}$ from an $\mathrm{OV}$ star or group or stars with luminosity $\sim 10^{6} L \odot$ (providing the radiation pressure on the grains), local gas density $\sim 10^{4} \mathrm{~cm}^{-3}$, temperature $\sim 10^{4} \mathrm{~K}$, plasma $\beta \sim 10, t_{\mathrm{s}}$ calculated including both Epstein and Coulomb drag terms, and the grain charge calculated including photoelectric and collisional charging in that radiative environment (and accounting for saturation of the grain charge). H II-far takes the same system, and re-calculates all properties assuming a distance $r \sim 1 \mathrm{pc}$ from the star (assuming the gas density falls $\propto r^{-2}$ and $\beta$ is constant). The important difference, for our purposes, is that the equilibrium drift velocity $\mathbf{w}_{\mathrm{s}}$ is supersonic in H II-near (where the radiation field is stronger), and subsonic in H II-far.

Likewise AGB is chosen to represent grains near the base (at $r \sim 100 R_{\odot}$ ) of a dust-driven wind from a cool giant star, with a steady-state wind mass-loss rate $\dot{M}_{w} \sim 10^{-6} \mathrm{M}_{\odot} \mathrm{yr}^{-1}$, wind velocity $v_{w} \sim 10 \mathrm{~km} \mathrm{~s}^{-1}$, temperature $T \sim 2000 \mathrm{~K}, \beta \sim 1$, stellar luminosity $\sim 10^{3} L_{\odot}$ providing the grain acceleration, and $\sim 0.1 \mu \mathrm{m}$ grains with a similar calculation of the charge and drag parameters. The distinguishing feature of this case is that the high gas densities $\left(\sim 10^{12} \mathrm{~cm}^{-3}\right)$ mean drag (collisional) grain coupling strongly dominates over Lorentz forces, so $\tau \ll 1$. $^{4}$

${ }^{4}$ As shown in Paper I, at low $\tau \ll 1$, the gyro RDIs are formally present, but are generally stabilized, except at either very large $k$ or very specific angles
WIM represents a random patch of the diffuse warm ISM, with $\beta \sim 1, T \sim 10^{4} \mathrm{~K}$, gas density $\sim 1 \mathrm{~cm}^{-3}$. We assume that the radiation energy density accelerating grains is comparable to the thermal energy density. Here $\tau \gg 1$ because the gas is diffuse, and the drift is highly subsonic.

Corona has parameters similar to those expected near the base of the solar Corona: $r \sim R_{\odot}, L \sim L_{\odot}$ (with both gravity and radiation contributing to the drift), $n \sim 10^{8} \mathrm{~cm}^{-3}, \beta \sim 0.001, T \sim 10^{6} \mathrm{~K}$, for $\sim 0.1 \mu \mathrm{m}$ grains. Here $\beta \ll 1$ makes this regime distinct; this also means the drift is supersonic but sub-Alfvénic, and $\tau \gg 1$.

CGM represents parameters that could be present in the CGM at $r \sim 100 \mathrm{kpc}$ from a bright quasar with $L \sim 10^{13} L_{\odot}$, and $n \sim$ $10^{-2} \mathrm{~cm}^{-3}, T \sim 10^{6} \mathrm{~K}, \beta \sim 1000$. The low density means $\tau \sim 10^{3}-$ $10^{7}$ is large, while the high luminosity provides a super-Alfvénic equilibrium drift, producing a distinct mode structure.

Example is not chosen to match a particular system, but the case is examined in Seligman et al. (2019), including a resolution study. It lies between H II-near and WIM, so we include it here for comparison.

For each of these parameter sets, Fig. 2 shows the linear growth rates of the instabilities as a function of wavelength. For a given $k=|\mathbf{k}|$, these are calculated by choosing a mode angle $\hat{\mathbf{k}}$, which is either parallel to the drift or magnetic field, or satisfies one of the various resonant conditions at which a natural dust oscillation frequency matches one of the gas (e.g. dust advection and Alfvén waves). For each class of resonance, we plot the maximum growth rate for each $\hat{\mathbf{k}}$. More information, including how to calculate the resonant angles, is given in Paper I.

Although all scales are unstable, different modes have growth rates that depend on the scale differently. In the physical systems explored above, the dynamic range between the largest global scales (where our local box treatment would be inappropriate) and the smallest scales where the instability operates is so large that it is impossible to resolve in a single box. Therefore, we construct a series of boxes for most cases here, ${ }^{5}$ each of which resolves a different range of wavelengths.

The parameters presented above are plausible, but will vary within and between different astrophysical regimes. Parameters that depend on highly uncertain grain chemistry or physical structure, such as $\tau$ (which depends on the grain charge), are particularly uncertain. Other quantities held fixed in our study - e.g. the gas equation of state, or the dust-to-gas ratio - might vary between regimes. For this reason we consider a number of variations in gas and dust physics compared to the 'default' simulations. These are noted in Table 2 and discussed in detail in Section 4.2.

The simulations presented here are scale free, and can represent any system that has the same dimensionless parameters. They are not strictly tied to the one specific physical system described above, but rather provide a well-motivated starting point for our study. For example, as discussed in Paper I, at different stages in the evolution of a supernova remnant (SNR), the SNR will pass through extended periods with parameters broadly similar to the AGB, Corona, and H II-near regimes above (although details will differ). Similarly, many regions of GMCs and the obscuring dusty torus around AGN will feature parameters resembling the AGB case. Some further

where they become degenerate with the MHD-wave RDIs. For this reason we do not plot them in Fig. 1 for the AGB case.

${ }^{5}$ We only consider a single box size in Example, as the results resemble $\mathrm{H}$ II-far, and CGM, as the mode structure does not change over a large range of wavelengths. 

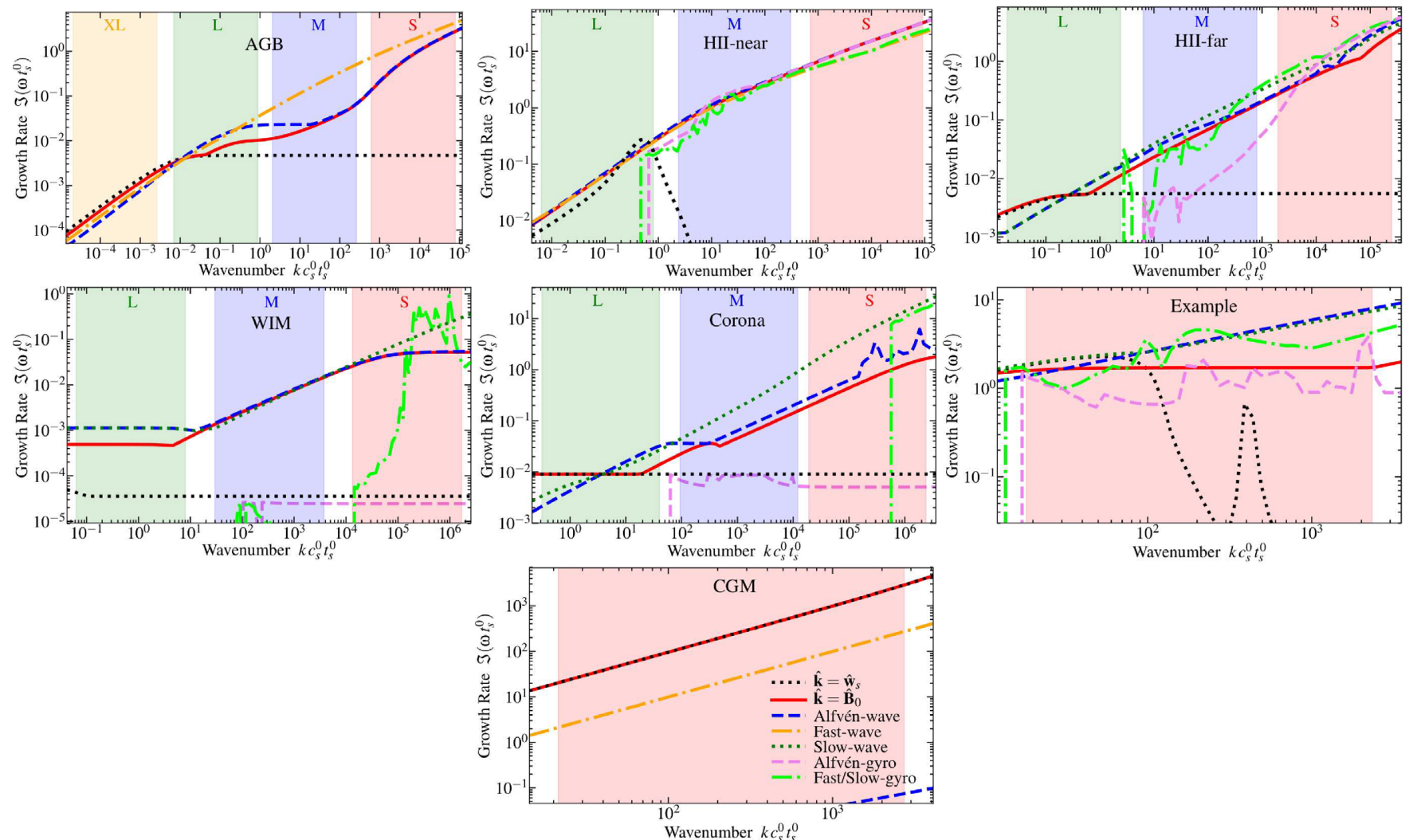

Figure 2. Maximal growth rates predicted by linear theory for different modes in the simulations. Each panel corresponds to one simulation set from Table 1 and Fig. 1 (fixed $\tau, \mu, \beta,\left|\hat{\mathbf{B}}_{0} \cdot \hat{\mathbf{a}}\right|,\left|\mathbf{w}_{\mathrm{s}}^{0}\right| / c_{\mathrm{s}}^{0}, \gamma$, etc.), with shaded regions showing the range of wavelengths covered by each simulation box (S/M/L) with different $L_{\mathrm{box}} / c_{\mathrm{s}}^{0} t_{\mathrm{s}}^{0}$. We plot predicted linear growth rates versus wavenumber $k=|\mathbf{k}|=2 \pi / \lambda$. Different lines correspond to different mode directions $\hat{\mathbf{k}}$ : parallel to the drift $\left(\hat{\mathbf{k}}=\hat{\mathbf{w}}_{s}\right)$, parallel to the magnetic field $(\hat{\mathbf{k}}=\hat{\mathbf{B}})$, and directions which satisfy the conditions for different RDIs (the Alfvén-wave, slow and fast magnetosonic-wave RDIs, and the Alfvén-gyro and slow/fast-gyro RDIs). For the RDIs, we plot the maximum growth rate marginalized over mode angles $(\hat{\mathbf{k}})$ which satisfies the resonant condition at each $k$. Note the fast-wave RDI can only exist if $\left|\mathbf{w}_{\mathrm{s}}^{0}\right| / c_{\mathrm{s}}^{0}>1$, and the gyro RDIs only exist above some $k$ and $\tau$ (slow and fast-gyro RDIs have degenerate solutions so are plotted as one line) ${ }^{4}$ - see Paper I for details. Generically, all wavelengths are unstable, and smaller scale modes grow faster, although which modes dominate is scale-and-parameter dependent.

intuition can be gained from Fig. 1 (although note that there are other important parameters that cannot be easily shown on a twodimensional plot)

We broadly classify the behaviour of the saturated simulations into two regimes. The first is the 'clumped' regime, where the dust becomes strongly concentrated; this occurs, for example, in H IInear (Fig. 4), H II-far (Fig. 5), Example (Fig. 16), and AGB (Fig. 6). The second is the 'disperse' regime, where the dust is expelled from certain regions at high velocity but remains relatively homogeneous; this occurs in (some scales in) WIM (Fig. 7), CGM (Fig. 13), and Corona (Fig. 8).

\section{RESULTS}

\subsection{Default simulation set}

We evolve each box well into its non-linear growth phase. As a first example, Fig. 3 shows visualizations ${ }^{6}$ of the dust and gas in

${ }^{6}$ The gas + dust visualization is constructed by interpolating the continuous gas properties on to the $x / y / z$ axis surfaces, and taking all dust 'superparticles' that lie within a thin slice of width equal to roughly the median interparticle separation and projecting them on to the surface. The dust-density visualization is constructed by first calculating the dust density in the vicinity of the large H II-near simulation box, at a time well into the non-linear phase of the evolution. Large fluctuations and coherent structure are visible in all gas quantities and in the dust.

Figs 4-8 show the morphologies of gas and dust in the default H II-near, H II-far, AGB, WIM, and Corona sets, respectively. We show gas and dust properties during two different simulation times, corresponding to the early non-linear and saturated phases of evolution. For each parameter set, we show the boxes of different sizes in parallel columns. Sequential simulations from left to rightare $\sim 300 \times$ larger in size scale; an entire box at the left is approximately $\sim 1 / 2$ of a pixel/element in the box to its immediate right. We only show the gas quantities that exhibit the most obvious morphological structure. Figs 11-13 and 19 show the same for the Example, CGM, and H II-near sets. Parallel columns now compare physical variations (e.g. changing the equation of state of the gas, or dust charge scaling). These physical variations are discussed further in Section 11.

each dust particle element using a kernel density estimator as in Moseley et al. (2019), then plotting each dust particle in the projected 3D space, colour coded by the density (with a constant transparency). The range on the colour bars is scaled to include some fraction (typically $>90$ per cent) of the plotted elements, in order to show contrast, but there are always some elements with higher/lower values. 
Table 2. Dispersion in various gas and dust quantities in the simulations, during the saturated state. We measure the rms dispersion $\delta X$ in quantity $X$, averaged over the last several snapshots in time for each run. We show gas velocity $\delta \mathbf{u}_{\mathrm{g}}^{x, y, z}$ in each direction (see Fig. 3 for axis convention), dust velocity $\delta \mathbf{v}_{\mathrm{d}}$, magnetic field $\delta \mathbf{B}$, gas density $\delta \ln \rho_{\mathrm{g}}$, and dust density $\delta \ln \rho_{\mathrm{d}}$. The dispersions shown are mass weighted for $\mathbf{u}_{\mathrm{g}}$ and $\mathbf{v}_{\mathrm{d}}$ (so that the kinetic energy of gas is just $(1 / 2) M_{\text {gas }}\left|\delta \mathbf{u}_{\mathrm{g}}\right|^{2}$, and likewise for dust), while dispersions for $\mathbf{B}$ and $\rho_{\mathrm{g}}, \rho_{\mathrm{d}}$ are volume weighted. Usually the mass and volume weights give similar values; where they differ substantially, the value in parenthesis gives the other (for more details, see Figs 21-29). For each parameter set, we list the 'default' boxes $\mathrm{S} / \mathrm{M} / \mathrm{L} / \mathrm{XL}$ as defined in Table 1, as well as the variants discussed in 4.2. For Example, we show variations in $\beta, \gamma$, and $\mu$ from Figs 11 and 16 . For H II-near L, we compare variations in the dust charge ('L: $\tau=10$ '; $q_{\text {grain }}$ increased by a factor $\sim 4$ ), as well as different charging models, including un-saturated collisional charging ('L:CC', $q_{\text {grain }} \propto T$ with $\gamma=5 / 3$ ) and photoelectric charging ('L:PE'; $q_{\text {grain }} \propto T / \rho^{1 / 2}, \gamma=1$ ), from Fig. 12. We also compare variations in the angle, $\mathbf{B}_{0} \cdot \mathbf{a}=\cos \theta_{\mathbf{B a}}$ with $\theta_{\mathbf{B a}}=\left(70^{\circ}, 20^{\circ}\right)\left({ }^{\circ} \mathrm{L}: 70^{\circ},{ }^{\prime} \mathrm{L}: 20^{\circ}\right.$, respectively) from Fig. 15, and different dust-to-gas ratios $\mu$ from Fig. 18. For WIM, we compare variations of $\mathrm{S} / \mathrm{M} / \mathrm{L}$ with $5 \times$ lower $\mathbf{a}$ and $\mathbf{w}_{\mathrm{S}}$ ('LoV') from Fig. 14. For CGM, we show runs with lower $\tau$ ( $\tau \approx 4500$ implying $q_{\text {grain }}$ is $\sim 30 \times$ lower; labelled ' $\tau_{\text {low' }}$ '), and runs with un-saturated photoelectric (PE) charging and $\gamma=5 / 3$ (for both the default and low $\tau$ cases), from Fig. 13.

\begin{tabular}{|c|c|c|c|c|c|c|c|c|c|c|c|}
\hline Name & $\frac{\delta \mathbf{u}_{\mathrm{g}, x}}{c_{\mathrm{S}}^{0}}$ & $\frac{\delta \mathbf{u g}_{\mathrm{g}, y}}{c_{\mathrm{s}}^{0}}$ & $\frac{\delta \mathbf{u g}_{,}, z}{c_{\mathrm{s}}^{0}}$ & $\frac{\delta \mathbf{v}_{\mathrm{d}, x}}{c_{\mathrm{s}}^{0}}$ & $\frac{\delta \mathbf{v}_{\mathrm{d}, y}}{c_{\mathrm{s}}^{0}}$ & $\frac{\delta \mathbf{v}_{\mathrm{d}, z}}{c_{\mathrm{s}}^{0}}$ & $\frac{\delta \mathbf{B}_{x}}{\left(4 \pi P_{0}\right)^{1 / 2}}$ & $\frac{\delta \mathbf{B}_{y}}{\left(4 \pi P_{0}\right)^{1 / 2}}$ & $\frac{\delta \mathbf{B}_{z}}{\left(4 \pi P_{0}\right)^{1 / 2}}$ & $\delta \ln \left[\frac{\rho_{\mathrm{g}}}{\rho_{\mathrm{g}}^{0}}\right]$ & $\delta \ln \left[\frac{\rho_{\mathrm{d}}}{\rho_{\mathrm{d}}^{0}}\right]$ \\
\hline \multicolumn{12}{|l|}{ Example: } \\
\hline Default & 0.075 & 0.025 & 0.017 & 0.086 & 0.063 & 0.32 & 0.075 & 0.025 & $6.4 \mathrm{e}-3$ & 0.013 & 1.2 \\
\hline$\beta=1$ & 0.067 & 0.015 & $6.6 e-3$ & 0.095 & 0.086 & 0.20 & 0.067 & 0.013 & $3.1 \mathrm{e}-3$ & $3.7 \mathrm{e}-3$ & $1.7(1.4)$ \\
\hline$\gamma=5 / 3$ & 0.088 & 0.039 & 0.020 & 0.12 & 0.11 & 0.33 & 0.089 & 0.037 & 0.013 & $8.2 \mathrm{e}-3$ & $1.2(1.3)$ \\
\hline$\mu=1 \mathrm{e}-3$ & $4.3 e-3$ & $1.8 \mathrm{e}-3$ & $6.7 \mathrm{e}-3$ & $2.6 \mathrm{e}-3$ & $3.2 \mathrm{e}-3$ & 0.035 & $5.1 \mathrm{e}-3$ & $1.8 \mathrm{e}-3$ & $4.1 \mathrm{e}-3$ & $4.9 \mathrm{e}-3$ & 2.7 \\
\hline$\mu=0.1$ & 0.18 & 0.15 & 0.11 & 0.32 & 0.30 & 0.41 & 0.17 & 0.12 & 0.056 & 0.055 & 0.89 \\
\hline \multicolumn{12}{|l|}{ AGB: } \\
\hline S & 0.052 & 0.050 & 0.014 & $6.9 e-4$ & $9.4 \mathrm{e}-4$ & $7.6 e-4$ & $8.6 e-3$ & $9.4 \mathrm{e}-3$ & 0.049 & 0.052 & 0.75 \\
\hline M & 0.031 & 0.010 & 0.038 & 0.10 & $6.8 \mathrm{e}-3$ & 0.11 & 0.066 & $4.2 \mathrm{e}-3$ & 0.071 & 0.074 & 0.90 \\
\hline $\mathrm{L}$ & 1.0 & 0.50 & 1.2 & 1.7 & 0.50 & 1.9 & 1.2 & 0.51 & 1.2 & 0.85 & 0.88 \\
\hline XL & 19 & 4.3 & 18 & 20 & 3.2 & 20 & 11 & 2.6 & 12 & 1.1 & 1.1 \\
\hline \multicolumn{12}{|l|}{ H II-near: } \\
\hline S & 0.060 & 0.057 & 0.013 & $3.5 \mathrm{e}-3$ & $3.6 \mathrm{e}-3$ & $3.7 e-3$ & $5.1 \mathrm{e}-3$ & $4.7 \mathrm{e}-3$ & 0.023 & 0.080 & 1.2 \\
\hline M & 0.15 & 0.13 & 0.20 & 0.76 & 0.80 & 0.65 & 0.15 & 0.11 & 0.14 & 0.058 & 1.4 \\
\hline $\mathrm{L}$ & 3.3 & 1.8 & 3.6 & 13 & $15(31)$ & 12 & 3.7 & 1.9 & 3.5 & 1.2 & $1.5(2)$ \\
\hline $\mathrm{L}: \tau=10$ & $4.7(5.5)$ & $1.6(2.1)$ & $4.8(5.7)$ & $9.9(8.0)$ & $3.0(3.3)$ & $10(8.6)$ & 4.1 & 1.9 & 4.0 & $1.3(1.2)$ & $2.0(2.4)$ \\
\hline $\mathrm{L}: \mathrm{CC}$ & $2.9(13)$ & $2.6(12)$ & 2.7 (12) & $29(200)$ & $30(200)$ & $26(170)$ & 2.5 & 1.6 & 2.6 & $1.6(0.9)$ & $1.8(2.5)$ \\
\hline $\mathrm{L}: \mathrm{PE}$ & $6.9(60)$ & $7.0(61)$ & $6.3(51)$ & $80(1200)$ & $81(1200)$ & $63(940)$ & 4.3 & 4.0 & 5.0 & $2.0(1.1)$ & $2.2(2.2)$ \\
\hline $\mathrm{L}: 70^{\circ}$ & $7.2(8.5)$ & $2.1(2.4)$ & $4.7(5.7)$ & $15(11)$ & $3.4(3.1)$ & $8.5(7.4)$ & 4.8 & 2.2 & 3.0 & 1.2 & 2.2 \\
\hline $\mathrm{L}: 20^{\circ}$ & $2.8(3.1)$ & $1.5(1.9)$ & $7.1(7.7)$ & $4.4(4.2)$ & $2.7(2.9)$ & $10.7(9.5)$ & 3.0 & 1.7 & 6.8 & 1.3 & $1.9(2.5)$ \\
\hline $\mathrm{L}: \mu=1 \mathrm{e}-3$ & 1.1 & 0.36 & 1.2 & $3.0(2.3)$ & 1.2 & $3.2(2.5)$ & 0.88 & 0.28 & 0.95 & 0.56 & $2.4(3.2)$ \\
\hline $\mathrm{L}: \mu=0.1$ & $14(17)$ & $3.4(5.6)$ & $14(17)$ & $15(24)$ & $7.4(24)$ & $15(22)$ & 12 & 3.5 & 12 & 1.7 & 1.7 \\
\hline \multicolumn{12}{|l|}{ H II-far: } \\
\hline S & $1.8 \mathrm{e}-3$ & $1.8 \mathrm{e}-3$ & $2.0 \mathrm{e}-3$ & $4.7 e-5$ & $1.4 \mathrm{e}-5$ & $2.1 \mathrm{e}-5$ & $1.4 \mathrm{e}-3$ & $1.4 \mathrm{e}-3$ & $1.4 \mathrm{e}-3$ & $4.3 e-4$ & 0.63 \\
\hline M & $2.9 \mathrm{e}-3$ & $2.7 \mathrm{e}-3$ & $4.5 \mathrm{e}-3$ & $1.5 \mathrm{e}-3$ & $1.6 \mathrm{e}-3$ & $3.5 \mathrm{e}-3$ & $2.4 \mathrm{e}-3$ & $1.9 \mathrm{e}-3$ & $2.3 e-3$ & $7.4 \mathrm{e}-4$ & $1.3(2.5)$ \\
\hline $\mathrm{L}$ & 0.55 & 0.20 & 0.58 & 1.1 & 0.90 & 1.0 & 0.43 & 0.19 & 0.44 & 0.28 & $1.5(2.2)$ \\
\hline \multicolumn{12}{|l|}{ WIM: } \\
\hline $\mathrm{S}^{*}$ & $9.8 \mathrm{e}-4$ & $9.8 \mathrm{e}-4$ & $1.1 \mathrm{e}-3$ & $6.4 \mathrm{e}-4$ & $6.4 \mathrm{e}-4$ & $9.4 \mathrm{e}-6$ & $9.7 \mathrm{e}-4$ & $9.7 \mathrm{e}-4$ & $1.1 \mathrm{e}-3$ & $7.2 \mathrm{e}-4$ & 0.49 \\
\hline S:LoV & $9.8 \mathrm{e}-4$ & $9.8 \mathrm{e}-4$ & $1.1 \mathrm{e}-3$ & 7.0e-4 & $7.0 \mathrm{e}-4$ & $4.3 e-6$ & $9.7 e-4$ & $9.7 \mathrm{e}-4$ & $8.6 e-4$ & $7.2 \mathrm{e}-4$ & 0.42 \\
\hline M & $9.9 e-4$ & $9.8 \mathrm{e}-4$ & $1.1 \mathrm{e}-3$ & $1.4 \mathrm{e}-3$ & $1.4 \mathrm{e}-3$ & $1.2 \mathrm{e}-4$ & $9.7 e-4$ & $9.7 e-4$ & $9.4 \mathrm{e}-4$ & $7.2 \mathrm{e}-4$ & 0.36 \\
\hline M:LoV & $1.0 \mathrm{e}-3$ & $1.0 \mathrm{e}-3$ & $1.1 \mathrm{e}-3$ & $6.0 \mathrm{e}-4$ & $6.0 \mathrm{e}-4$ & $2.0 \mathrm{e}-4$ & $9.7 \mathrm{e}-4$ & $9.7 e-4$ & $8.5 \mathrm{e}-4$ & $7.3 e-4$ & 0.25 \\
\hline $\mathrm{L}$ & 0.032 & 0.021 & 0.088 & 5.3 & 5.3 & 0.085 & $3.6 e-3$ & $3.6 e-3$ & $3.9 \mathrm{e}-3$ & $3.5 \mathrm{e}-3$ & 0.37 \\
\hline L:LoV & $1.5 \mathrm{e}-3$ & $1.3 e-3$ & $2.3 e-3$ & $1.1 \mathrm{e}-3$ & $7.3 e-3$ & $1.2 \mathrm{e}-3$ & $1.1 \mathrm{e}-3$ & $1.1 \mathrm{e}-3$ & $8.0 \mathrm{e}-4$ & $8.0 \mathrm{e}-4$ & 0.25 \\
\hline \multicolumn{12}{|l|}{ Corona: } \\
\hline $\mathrm{S}^{*}$ & 0.011 & 0.011 & 0.013 & 0.012 & 0.012 & $3.5 e-5$ & 0.011 & 0.011 & 0.013 & 0.060 & 0.63 \\
\hline M & 0.012 & 0.012 & 0.014 & 0.62 & 0.63 & 0.010 & 0.011 & 0.011 & 0.022 & 0.060 & 0.86 \\
\hline $\mathrm{L}$ & 0.27 & 0.24 & 0.086 & 24 & 24 & 3.1 & 0.076 & 0.069 & 0.15 & 0.066 & 0.57 \\
\hline $\mathrm{L}: \tau=100$ & 0.14 & 0.05 & 0.21 & 0.17 & 0.13 & 0.21 & 0.023 & 0.023 & 0.013 & 0.085 & $0.6(0.8)$ \\
\hline \multicolumn{12}{|l|}{ CGM: } \\
\hline Default & 0.83 & 0.82 & 0.63 & 23 & 23 & 22 & 0.040 & 0.040 & 0.042 & 0.34 & 0.28 \\
\hline $\mathrm{C}=1.07 \gamma=5 / 3+\mathrm{PE}$ & 2.5 & 2.5 & 2.0 & 63 & 63 & 63 & 0.14 & 0.14 & 0.13 & 0.56 & 0.25 \\
\hline $\mathrm{C}=1.06 \tau_{\text {low }}$ & 0.031 & 0.031 & 0.036 & 2.0 & 2.0 & 1.9 & 0.024 & 0.021 & 0.019 & 0.021 & 0.27 \\
\hline$\tau_{\text {low }}+\mathrm{PE}$ & 0.047 & 0.046 & 0.057 & 2.9 & 2.9 & 2.9 & 0.035 & 0.032 & 0.023 & 0.025 & 0.27 \\
\hline
\end{tabular}

Fig. 9 shows the magnitude of fluctuations in dust velocity versus time for each simulation. All dust fluctuations are plotted in units of the expected linear theory growth time-scales, which allows us to compare all of the simulations on the same time axis. Since there is no single growth time in a simulation (see Fig. 2), we consider the maximum growth rate of modes at the box scale. ${ }^{7}$ In most cases, modes near the resolution scale $\left(L_{\text {box }} / N\right)$ grow $\sim 10 \times$ faster than those at the box scale, ${ }^{8}$ and so the initial rapid growth is dominated by these small-scale modes. However, these modes generally saturate at a lower amplitude, so the later growth is then dominated by the box-scale modes, at rates in agreement with linear theory. This is surprising given the ambiguity of defining the

\footnotetext{
${ }^{7}$ Specifically we find the minimum 'box scale growth time' $t_{\text {grow }}\left(L_{\text {box }}\right) \equiv$ $1 / \Im\left(\omega_{\max }\right)$ where $\omega_{\max }$ corresponds to the mode with $|\mathbf{k}|=2 \pi / L_{\text {box }}$ that has the largest positive value of $\Im(\omega)$ (marginalizing over direction $\hat{\mathbf{k}}$ ).
}

\footnotetext{
${ }^{8}$ This estimate is not always accurate and depends on details of the dispersion relation. For example, in some cases, a different mode starts to dominate at some mid-range scale.
} 

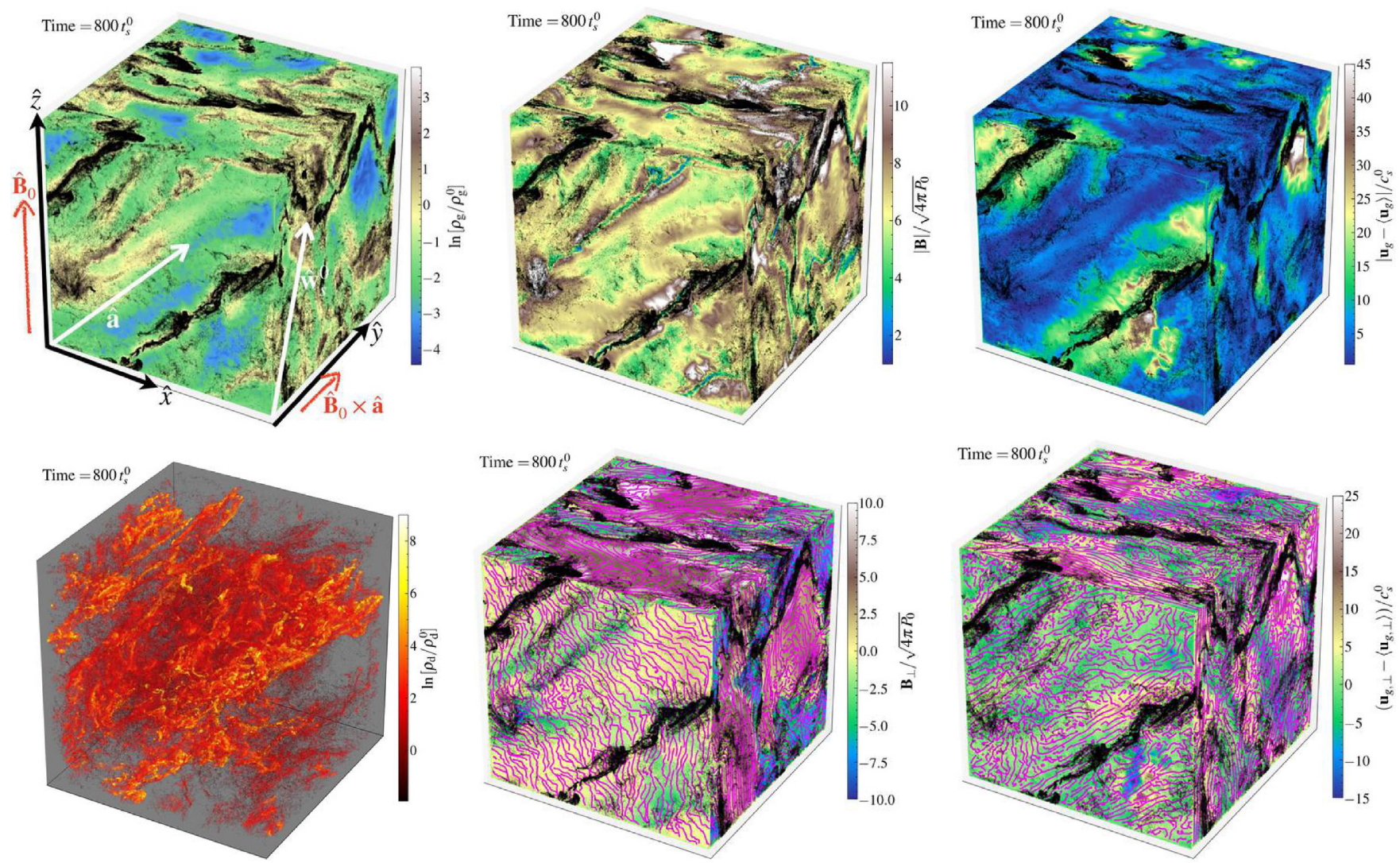

Figure 3. Images of our largest H II-near box (box L), from Table 1 and Fig. 2, after the instabilities reach saturation. Top: Surface projection of slices through each axis of the box, at the time labelled (time in units of the initial grain stopping time $\left.t_{\mathrm{s}}^{0}\right)$. Colours show gas quantities at the surface: $\rho_{\mathrm{g}}, \mathbf{B}, \mathbf{u}_{\mathrm{g}}(\mathrm{see}$ colour bar). Black points show dust grains on the surface. Surfaces are oriented so $\hat{\mathbf{B}}_{0}$ is the vertical axis $(\hat{z})$, $\hat{\mathbf{a}}$ is oriented in the $\hat{x}-\hat{z}$ direction (front or width-height plane), and the mutually perpendicular direction $\left(\hat{y} \propto \hat{\mathbf{B}}_{0} \times \hat{\mathbf{a}}\right)$ is depth. Bottom Left: 3D isometric plot of all grain particles (coloured by the local dust density), at the same time. Bottom middle and right: Surface plot of the vector $\mathbf{B}$ and $\mathbf{u}_{\mathrm{g}}$ : lines show the field or flow lines of the projected component on the surface (i.e. $\mathbf{B}_{x}$, $\mathbf{B}_{y}$ in the $x y$ plane) while colours show the magnitude of the normal component ( $\mathbf{B}_{z}$ in the $x y$ plane). The instabilities drive dramatic clumping/density structure in the dust and strong saturated Alfvénic turbulence in the gas. This occurs quickly relative to other time-scales: the time plotted is just $\approx 0.9 L_{\text {box }} / v_{\mathrm{f}, 0}$ (where $v_{\mathrm{f}, 0}^{2} \equiv\left(c_{\mathrm{s}}^{0}\right)^{2}+\left(v_{\mathrm{A}}^{0}\right)^{2}$ is the fastest gas wavespeed), i.e. less than one sound or Alfvén box-crossing time.

total growth rate, and the fact that small-scale modes have already become non-linear. All of the boxes eventually reach saturation, with the fluctuations in all properties in a statistical steady state. The cases in Fig. 9 that continue off the plot have been evolved to longer times to ensure that they are approximately saturated. ${ }^{9}$

In Table 2, we provide the magnitude of the time-averaged saturated fluctuations ${ }^{10}$ in each component of $\mathbf{u}_{\mathrm{g}}, \mathbf{v}_{\mathrm{d}}, \mathbf{B}, \rho_{\mathrm{g}}, \rho_{\mathrm{d}}$.

\footnotetext{
${ }^{9}$ We have verified that all simulation quantities within a given simulation saturate on a similar time-scale, as shown in Fig. 9. Explicit demonstrations of this, as well as plots of the full PDFs of the salient quantities versus time, for representative simulations, are shown in both Moseley et al. (2019) and Seligman et al. (2019).

${ }^{10}$ We define the fluctuation $\delta X$ in quantity $X$ as the rms $(1 \sigma)$ deviation, i.e. $\delta X \equiv\left\langle(X-\langle X\rangle)^{2}\right\rangle^{1 / 2}$, where $\langle U\rangle \equiv\left(\int U \varpi d^{3} \mathbf{x}\right) /\left(\int \varpi d^{3} \mathbf{x}\right)$ and $\varpi(\mathbf{x})$ is a weight. For $\mathbf{u}_{\mathrm{g}}, \mathbf{v}_{\mathrm{d}}$, and $\mathbf{B}$, it is most physical to relate the fluctuations to the energy in each component. So, for $\mathbf{u}_{\mathrm{g}}$ we use the gas-mass-weighted average $\left(\varpi=\rho_{\mathrm{g}}\right.$, such that the total kinetic energy of fluctuations is $\left.(1 / 2) M_{\mathrm{gas}}^{\text {box }}\left|\delta \mathbf{u}_{g}\right|^{2}\right)$, for $\mathbf{v}_{d}$ we likewise use the dust-mass-weighted average ( $=\rho_{d}$, so dust kinetic energy is $(\mu / 2) M_{\text {gas }}^{\text {box }}\left|\delta \mathbf{v}_{d}\right|^{2}$ ), and for $\mathbf{B}$ we use the volume average ( $\varpi=1$, so magnetic energy is $V_{\text {box }}|\delta \mathbf{B}|^{2} / 8 \pi$ ). For $\rho_{\mathrm{g}}, \rho_{\mathrm{d}}$, and $\rho_{\mathrm{d}} / \rho_{\mathrm{g}}$ we quote the volume-averaged fluctuations $(\varpi=1)$.
}

Fig. 10 plots these statistically averaged quantities against one another in various forms, and against some expectations from quasilinear theory for the saturated state. This is discussed further in Section 5.

Figs 11-15, 19, and 20, consider further comparisons of different physics and parameter variations, as discussed below.

Figs 21-27 examine various statistics of each run in more detail, plotting the PDFs of fluctuations of different quantities in the saturated state. ${ }^{11}$

The differences between mass and volume weighting are discussed further below. These are all defined in the centre-of-mass (i.e. co-moving or freeaccelerating) frame.

${ }^{11}$ Given our Lagrangian numerical method, at our default simulation resolution, dust underdensities $\rho_{d} / \rho_{d}^{0} \ll 10^{-6}$ cannot be resolved, but these are much smaller than any plotted in Figs 21-27 and much smaller than the typical fluctuations in Table 2. There is no formal upper limit to the maximum resolved concentration, but the dust becomes increasingly overresolved relative to the gas at very large $\rho_{\mathrm{d}} / \rho_{\mathrm{g}} \gg 10^{6}-$ however this is precisely the regime where we expect other physics to dominate, discussed below. 

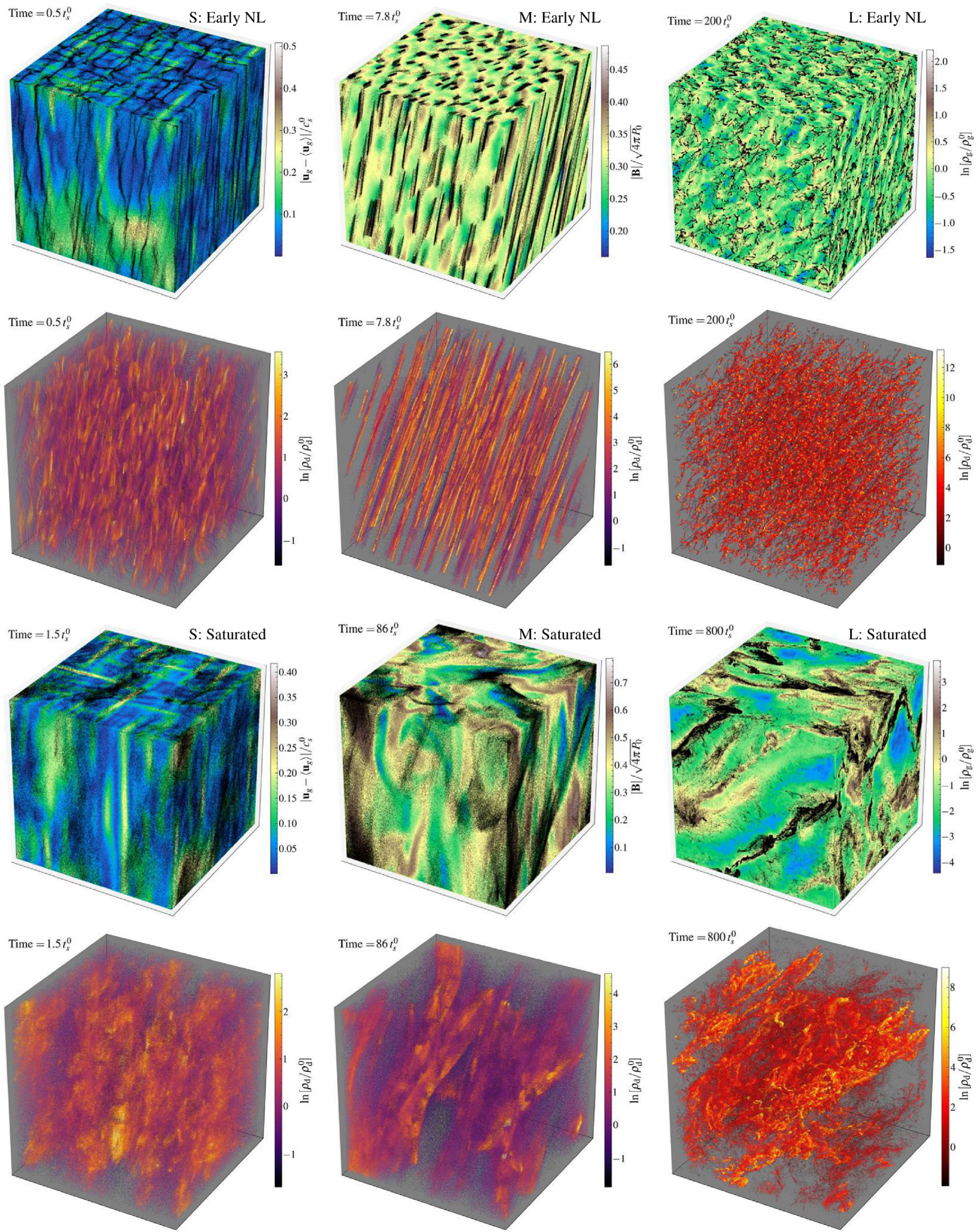

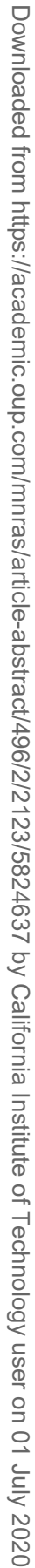

Figure 4. Images of H II-near, one of the default simulation 'sets' from Table 1 and Fig. 2. Columns show the three different box sizes S/M/L (increasing left to right). Top and second row: Gas + dust surface maps (top), and 3D dust maps (second), as Fig. 3, at time labelled (during early non-linear evolution). Third and bottom row: Same, but at a later time (in saturated state). Each box (moving left to right) is $\sim 300 \times$ larger than the previous (so an entire box at the left is approximately $\sim 1 / 2$ of a pixel/element in the box to its immediate right). Dust and turbulence clearly exhibit structure on all scales. Smaller scale modes drive weaker and less compressible gas turbulence, as predicted (Section 5). Slightly different physical parameters (compare Fig. 5), or different scales with the same parameters (boxes here) can produce wildly different morphologies (and resonant angles), even with different dimensionality of dust structures (e.g. point-like clumps, 1D filaments, 2D sheets). This owes to complicated wavelength dependence of the dominant modes (Fig. 2). The morphologies present in the L simulation (right-hand panels) provide an example of the 'clumped' saturation mode (see Section 5.3). 

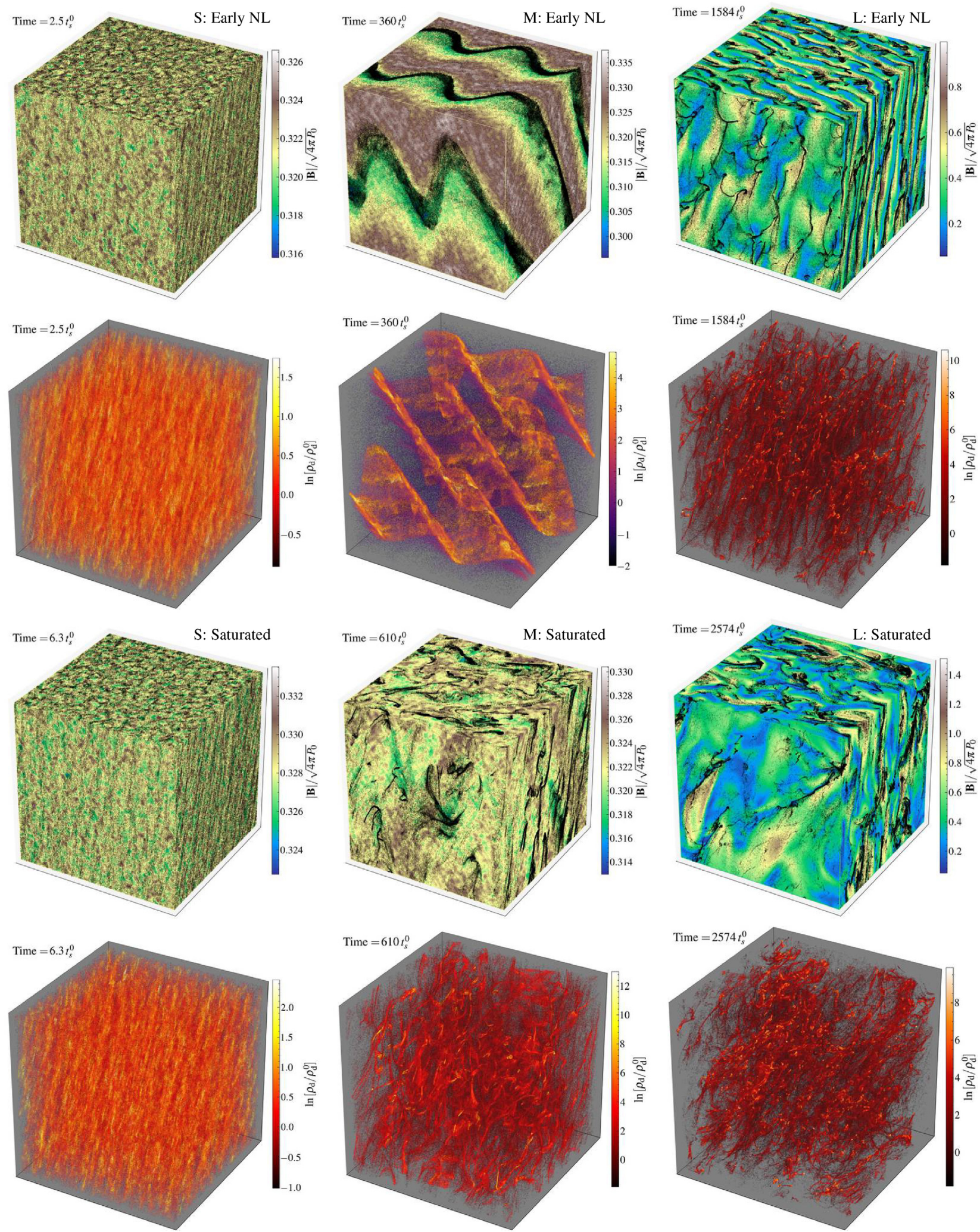

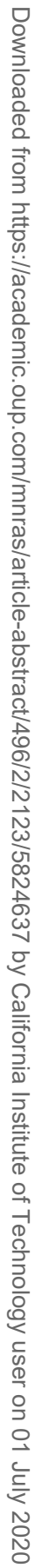

Figure 5. As Fig. 4, for H II-far. Despite the drift being subsonic (no 'fast mode' or acoustic resonances are possible), dramatic fluctuations still evolve on broadly similar time-scales. The dust exhibits strong clumping even in cases (S/M) where the gas is nearly incompressible. Different scales again exhibit distinct structure: $\mathrm{L}$ is dominated by the drift-aligned quasi-sound and pressure free modes (direction $\left.\hat{\mathbf{w}}_{\mathrm{s}}-\left(\hat{\mathbf{w}}_{\mathrm{s}} \cdot \hat{\mathbf{B}}\right) \hat{\mathbf{B}}\right) ;$ M by the slow and Alfvén MHD-wave modes (direction $\hat{\mathbf{B}}-\left(\hat{\mathbf{w}}_{\mathrm{S}} \cdot \hat{\mathbf{B}}\right) \hat{\mathbf{w}}_{\mathrm{s}}$ ), S by the slow-gyro modes (see Fig. 2). 

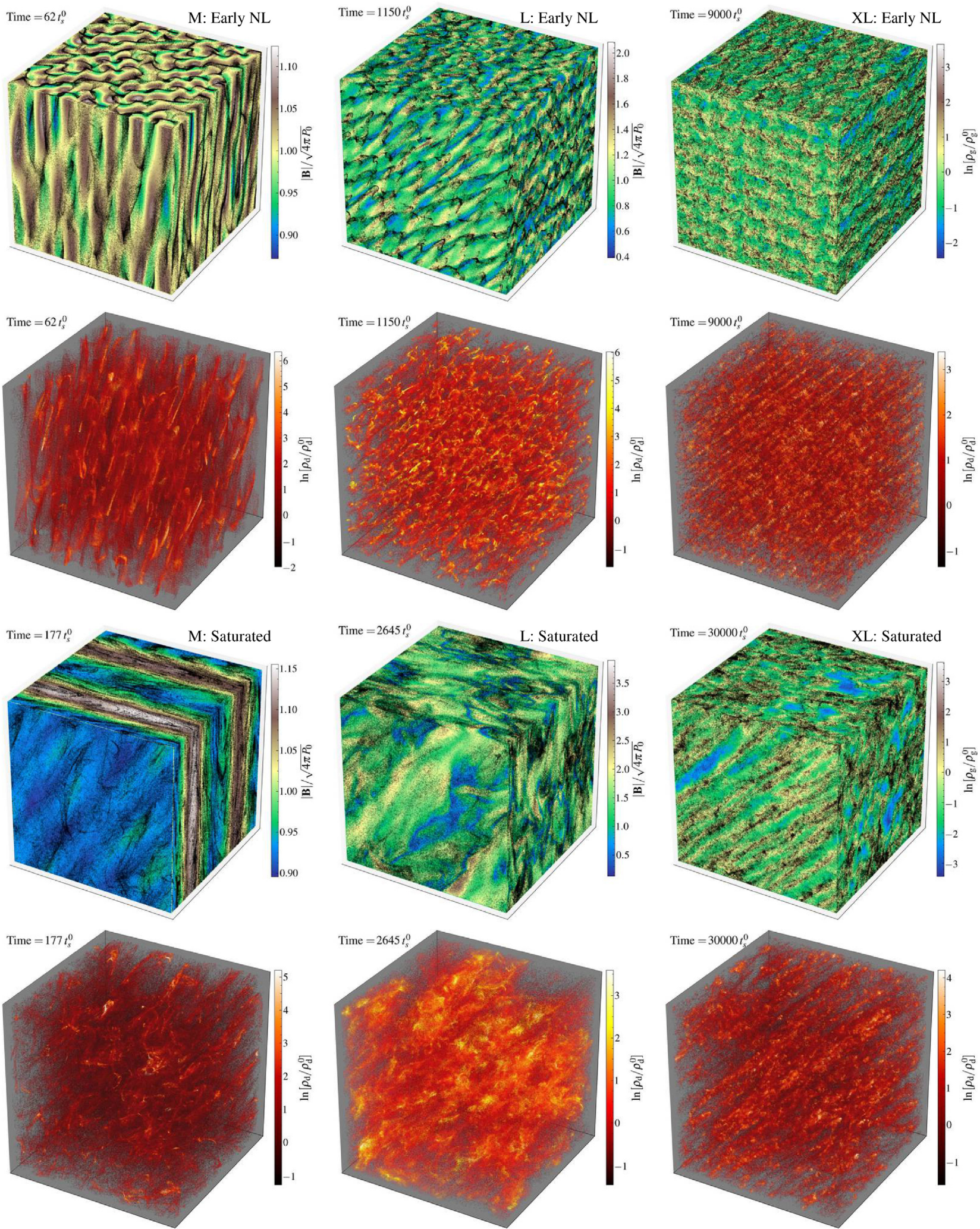

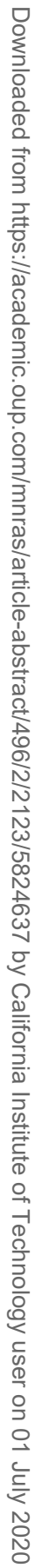

Figure 6. As Fig. 4, for AGB (M/L/XL shown; $\mathrm{S}$ is morphologically similar to H II-near $\mathrm{S}$ ). Because this initial condition features weak Lorentz forces on dust $(\tau \ll 1)$, the mode structure is simpler (dominated by the fast-MHD resonance at all $k$ ) and does not vary as dramatically with scale. Note here and in all similar figures, the colour bar for dust density only shows a fraction of the full dynamic range (empty regions reach lower densities, and small patches/clusters reach higher densities). 

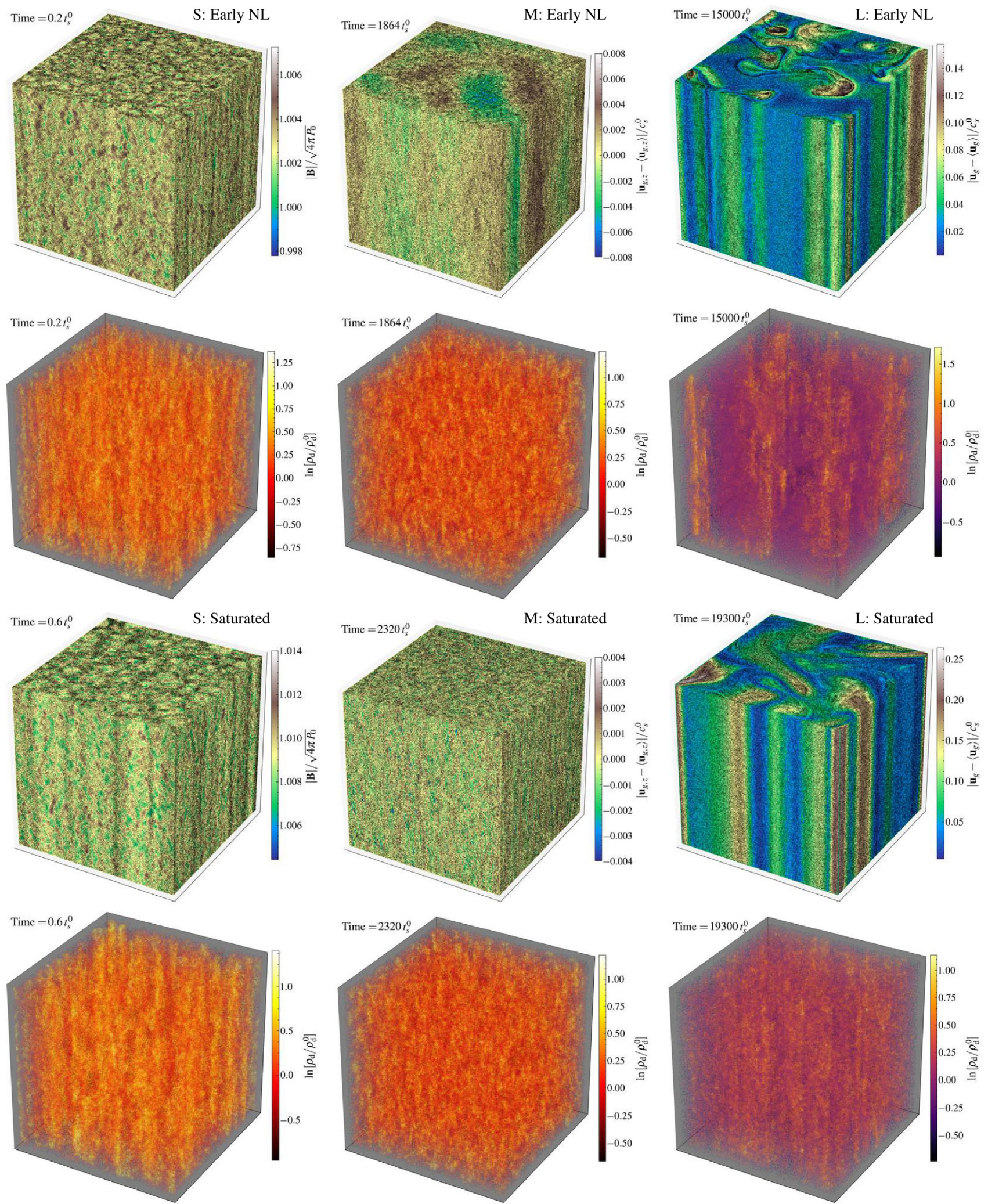

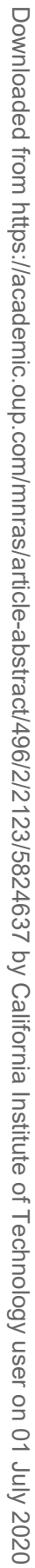

Figure 7. As Fig. 4, for WIM. In box S the strong slow-gyro resonance produces rapid growth of dust 'columns' parallel to the field, which collect in a 'granular' nature in the direction perpendicular to B. Box L features a slow-growing, almost-laminar mixing of large scale modes via the Alfvén resonance, with the dust confined along $\mathbf{B}$ (moving the $\mathbf{B}$ fields nearly incompressibe in the $x y$ plane). Box $\mathbf{M}$ features the weakest growth and saturated turbulence. 

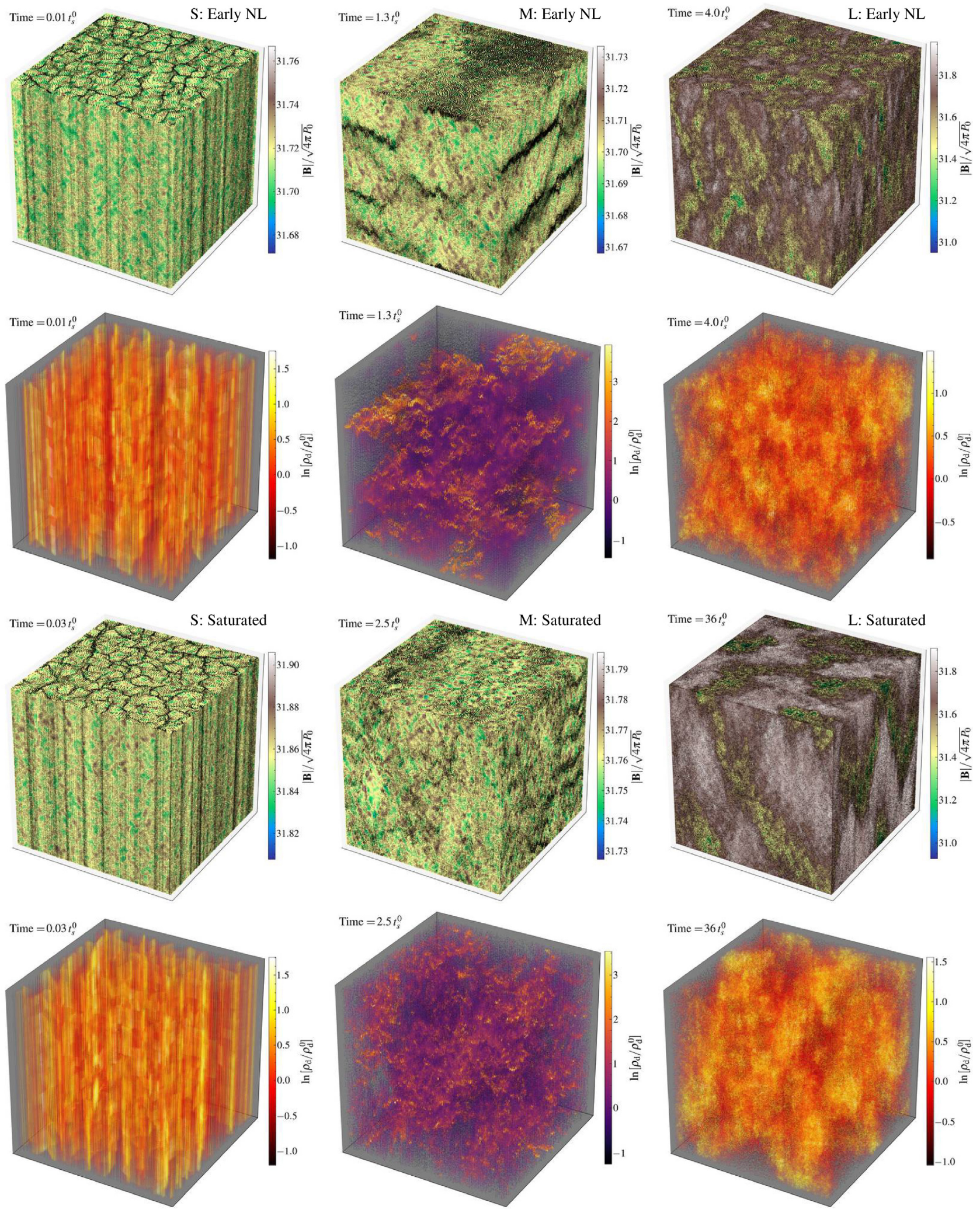

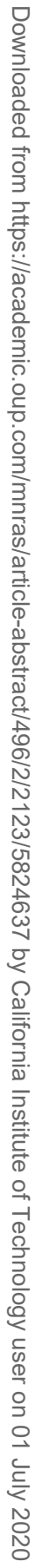

Figure 8. As Fig. 4, for Corona. The strong magnetization means almost all grain motion is tightly bound to field lines. This produces the $\hat{\mathbf{B}}$-aligned 'granular' structure in box S, and sheets of dust perpendicular to $\hat{\mathbf{B}}$ in M/L. Boxes M/L non-linearly transition to the 'disperse' mode (see Section 5.4), where the dust is driven to large isotropic velocity dispersions in the plane perpendicular to $\hat{\mathbf{B}}$ (representing large gyro orbits). 


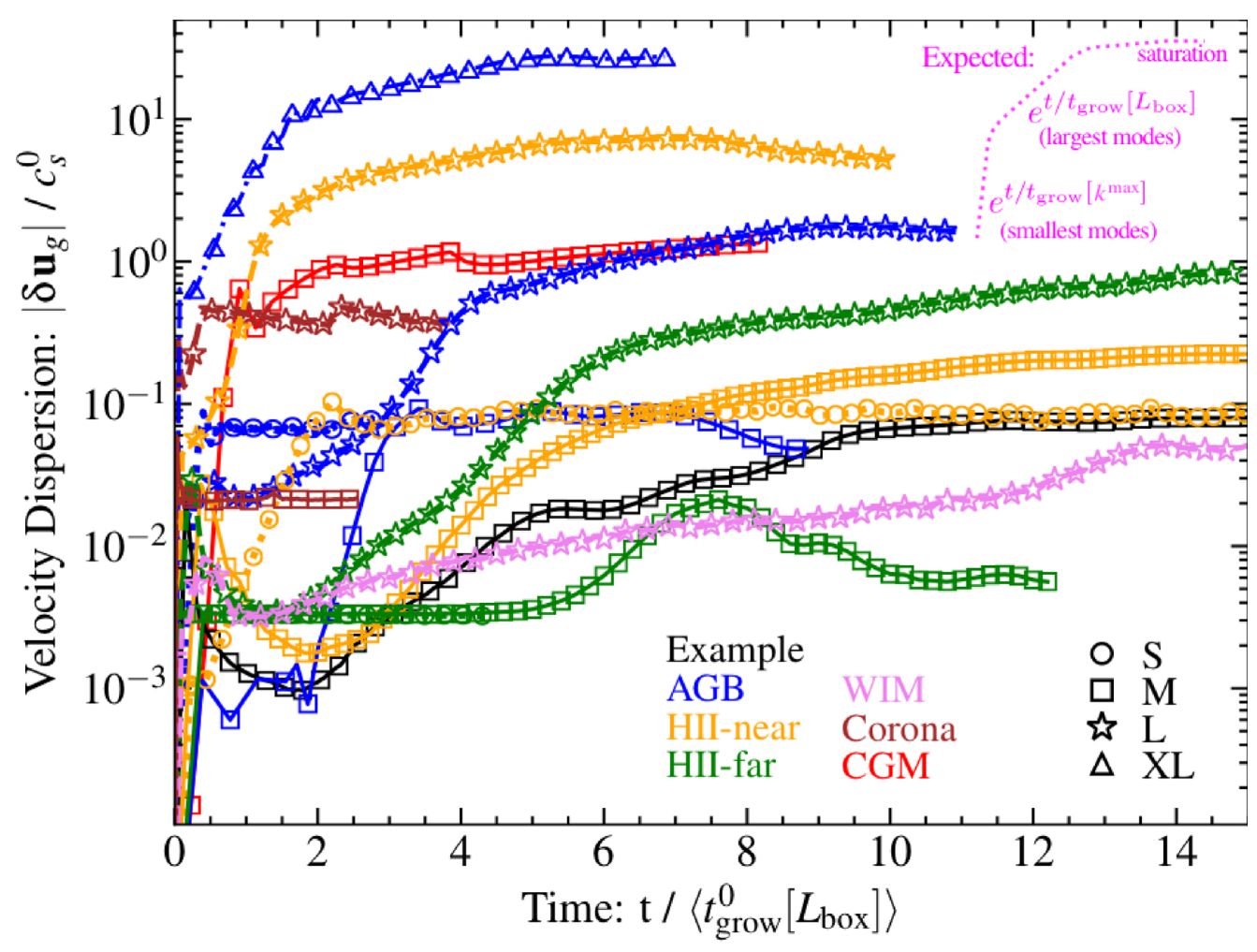

Figure 9. Instability growth versus time. We plot the (volume-weighted) gas velocity dispersion $\left(\left|\delta \mathbf{u}_{\mathrm{g}}\right|\right.$, in units of sound speed $c_{\mathrm{s}}^{0}$ ) as a function of time $(t)$ for boxes in Table 1 (lines with points; as labelled). Times $t$ are normalized by the analytic linear growth time of the fastest growing mode at the largest (box) scales: $\left\langle t_{\text {grow }}^{0}\left[L_{\text {box }}\right]\right\rangle \equiv 1 / \operatorname{MAX}\left(\omega\left[k=2 \pi / L_{\text {box }}\right]\right)$, calculated from the initial conditions at $|\mathbf{k}|=k^{\min }=2 \pi / L_{\text {box }}$ (see Fig. 2). The smallest modes in the box $(\sim 100 \times$ larger $k)$ typically have $\sim 10 \times$ larger growth rates $\left(t_{\text {grow }}\left[k^{\max }\right] \sim 0.1 t_{\text {grow }}\left[L_{\text {box }}\right]\right)$ : these are generally the fastest growing resolved modes and produce the initial rapid growth but saturate at smaller amplitude, so large-scale modes take over and grow at approximately the predicted rate (even though the box is often non-linear already), until global saturation. Magenta dotted line illustrates the predicted qualitative behaviour for this sequence. There are some exceptions, e.g. CGM where small-scale modes grow so violently non-linear that there is no obviously 'large-scale' mode domain. None the less, noting that $\left\langle t_{\text {grow }}^{0}\left[L_{\text {box }}\right]\right\rangle$ varies by factors $\gtrsim 10^{6}$ in the examples plotted, and that there is no unique growth rate for a given box (or even at a given $k$ ), we see surprisingly robust agreement with the linear predictions for both initial $\left(\sim k^{\max }\right.$-dominated) and late-time $\left(\sim k^{\mathrm{min}}\right.$-dominated) growth rates.

\subsection{Different equations of state, drag laws, and grain charge scalings}

In this subsection, we discuss the effects of additional physical variations in the simulations. We discuss the physical applicability of different drag laws in different regimes (expanding the discussion from Paper I), and explore different choices. We outline three idealized, physically motivated regimes of the grain charge scaling, and compare the effects of each of these in the simulations. We justify our usage of an isothermal equation of state for the majority of our simulations, and discuss astrophysical situations where different equations of state are appropriate. We also investigate how changes to the magnetic field, drift geometries, and additional grain parameters alters the non-linear evolution of the instabilities.

\subsubsection{Drag law}

The scalings and physical applicability of different drag laws are discussed in detail in Paper I. There it is shown that for any regime where the MHD RDIs are important, the drag is dominated by either Epstein or Coulomb drag (e.g. Stokes drag, important only when the grain is larger than the gas mean free path and Reynolds numbers are less than unity, is never relevant for the physical grain sizes in magnetized media considered here). Our default simulations adopt the Epstein drag scalings, which, for fixed grain properties, give

$t_{\mathrm{s}}^{-1} \propto \rho_{\mathrm{g}} c_{\mathrm{s}}\left(1+a_{E} w_{\mathrm{s}}^{2} / c_{\mathrm{s}}^{2}\right)^{1 / 2}($ Epstein $)$

$\left(a_{E}=9 \pi \gamma / 128\right)$. Likewise, Coulomb drag gives

$t_{\mathrm{s}}^{-1} \propto \rho_{\mathrm{g}} c_{\mathrm{s}}\left(1+a_{C} w_{\mathrm{s}}^{3} / c_{\mathrm{s}}^{3}\right) f(T, U)($ Coulomb $)$,

where $a_{C}=\left(2 \gamma^{3} / 9 \pi\right)^{3}$ and $f(T, U)=f_{\text {ion }}\left(z_{\mathrm{i}} U / T\right)^{2} \ln \Lambda$ is a function of the temperature $T$, ionization fraction $f_{\text {ion }}$, grain electrostatic potential $U$, gas ion charge $z_{\mathrm{i}}$, and a Coulomb logarithm $\ln \Lambda$.

It is difficult to evaluate Coulomb drag without specifying the physical system (to calculate $f_{\text {ion }}, T, \Lambda$, etc.), whereas Epstein drag is determined by scale free hydrodynamic quantities. Epstein drag dominates at high $w_{\mathrm{s}} / c_{\mathrm{s}}$, scaling as $\sim w_{\mathrm{s}} / c_{\mathrm{s}}$, while Coulomb drag becomes weaker, scaling as $\left(w_{\mathrm{s}} / c_{\mathrm{s}}\right)^{-3}$. Therefore, Epstein drag always dominates in supersonic cases (where $w_{\mathrm{s}} / c_{\mathrm{s}} \gtrsim 1$ or $\left.\left|\delta \mathbf{v}_{\mathrm{d}}\right| / c_{\mathrm{s}} \gtrsim 1\right)$. Moreover, Epstein drag often dominates in subsonic cases if the ionization fraction is small or if the grains reach charge saturation (see Paper I). We therefore expect Epstein drag to be a good approximation in many cases. Even in subsonic regimes where Coulomb drag may dominate, if the temperature and grain potential $U$ are fixed (as they are in our default runs), then Epstein and Coulomb drag scale identically (as $t_{\mathrm{s}}^{-1} \propto \rho$ ). Because the normalization of $t_{\mathrm{s}}$ is arbitrary (it simply goes into the dimensionless parameter $c_{\mathrm{S}} t_{\mathrm{s}} / L_{\text {box }}$ ), our predictions therefore apply equally well 

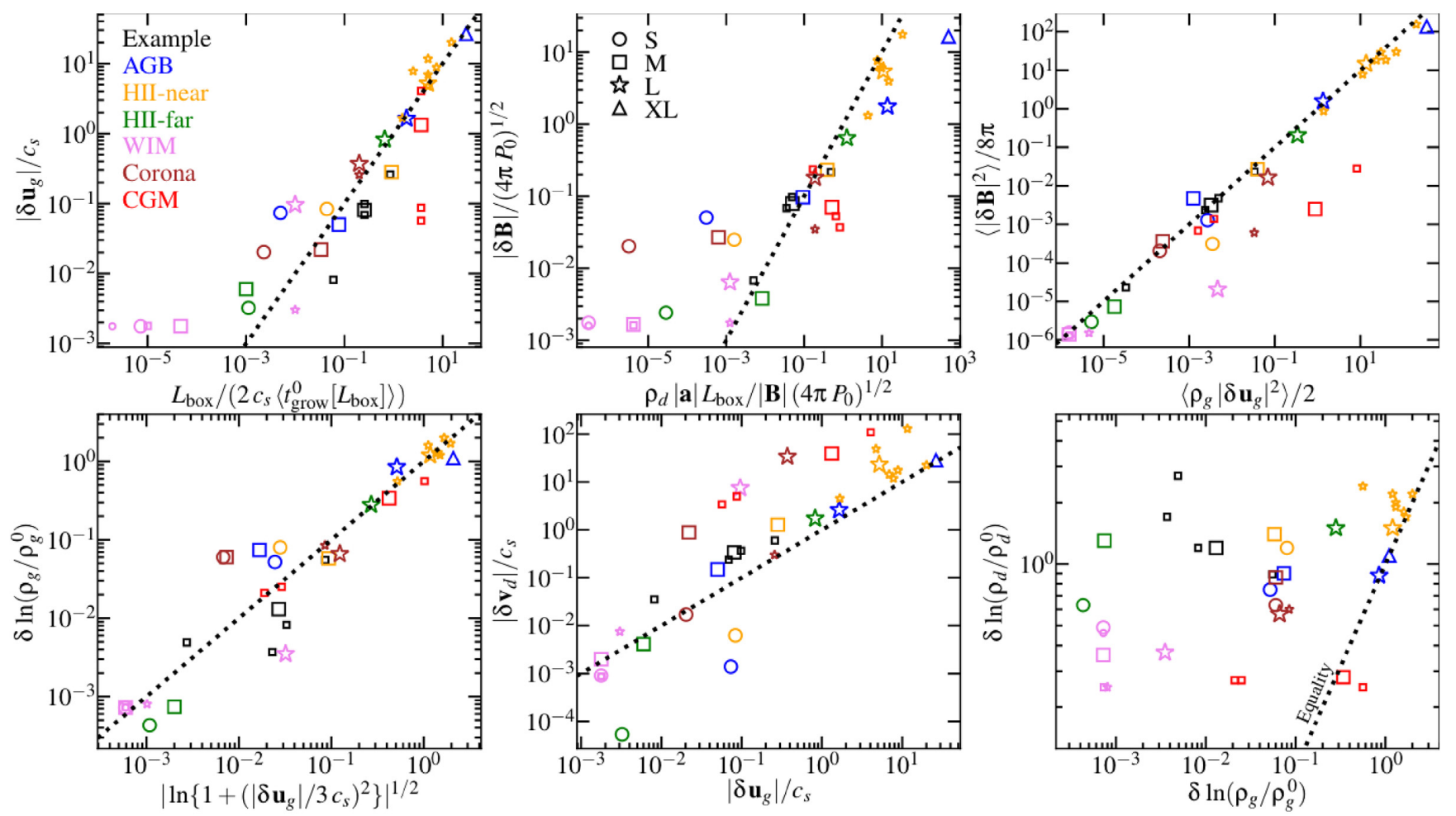

Figure 10. Correlations between the saturated fluctuations in gas and dust quantities. For each box (points as Fig. 9), we plot the fluctuations from Table 2, and compare simple analytic scalings (dotted lines). Small points denote the physics/parameter variations within each box from Table 2. Top left: Gas velocity dispersions versus those predicted from quasi-linear theory (equating eddy turnover and linear growth times at the box scale). We neglect anisotropy and just take $\left|\delta \mathbf{u}_{\mathrm{g}}\right|$; here $\omega\left(L_{\text {box }}\right)=1 / t_{\text {grow }}^{0}\left[L_{\text {box }}\right]$ as defined in Fig. 9. While imperfect (e.g. this systematically underpredicts the turbulence in the 'S' boxes) the scaling is order-of-magnitude plausible, with a couple notable outliers (CGM $\tau_{\text {low }}$ and WIM S/M), all of which are in the 'disperse' mode described in Section 5. Top middle: Magnetic fluctuations versus expectation if magnetic tension balances the force from gas on dust. this provides an order-of-magnitude plausible scaling but also with significant scatter and additional outliers. Top right: Kinetic and magnetic 'energy' of fluctuations (considering only the fluctuating terms). These agree to within a factor of $\sim 3$ over most of the dynamic range. Bottom left: Gas density versus velocity fluctuations. A scaling analogous to isothermal pure gas turbulence, $\delta \ln \left(\rho_{\mathrm{g}} / \rho_{\mathrm{g}}^{0}\right)=\sqrt{\ln \left[1+\left(b\left|\delta \mathbf{u}_{\mathrm{g}}\right| / c_{\mathrm{S}}\right)^{2}\right]}$ provides a reasonable fit, with the range $b \approx 0.2-1$. Bottom centre: Dust versus gas velocity fluctuations. There is a clear correlation, but with very large ( $\sim \pm 1$ dex) scatter. Usually $\delta \mathbf{v}_{\mathrm{d}}>\delta \mathbf{u}_{\mathrm{g}}$, with the notable exceptions of H II-far/near S and AGB S. Bottom right: Dust versus gas density fluctuations. There is essentially no correlation, except to require $\delta \rho_{\mathrm{d}}>\delta \rho_{\mathrm{g}}$. We see $\delta \rho_{\mathrm{d}}$ can be large even when the gas is nearly incompressible $\left(\delta \rho_{\mathrm{g}}<10^{-3}\right)$. See text for more discussion of each case (Section 5).

to either drag law in this subsonic regime, provided one re-scales the simulations dimensionless parameters appropriately.

\subsubsection{Grain charge}

Scalings of grain charge remain uncertain and depend on a variety of environmental and local factors (see Paper I and, e.g. Draine \& Sutin 1987; Weingartner \& Draine 2001a,b; Tielens 2005). However, if we assume that external environmental properties are fixed (e.g. an UV radiation field) and grain material properties are fixed (e.g. compositions, sizes), then three regimes emerge, which we define as

$Z_{\text {grain }} \propto \begin{cases}\text { constant } & \text { (a) } \\ T & \text { (b) } \\ T^{1 / 2} \rho_{\mathrm{g}}^{-1} & \text { (c). }\end{cases}$

(a) 'Fixed charge': if the grain charge is saturated (maxi$\mathrm{mal} / \mathrm{minimal}$ ), or the charge-aggregation/equilibration times are long compared to the time-scales we evolve, then the charge is approximately fixed. (b) 'Collisional charging': if the charging is dominated by collisional processes, is sufficiently fast, and is unsaturated, it depends solely on the temperature as $Z_{\text {grain }} \propto T$. (c) 'Pho- toelectric charging': if the charging is dominated by photoelectric processes (and again is also un-saturated and sufficiently rapid), then $Z_{\text {grain }} \propto T^{1 / 2} \rho_{\mathrm{g}}^{-1}$. Our default simulations assume regime (a), i.e. fixed charge, which is common for large grains in well-ionized environments. ${ }^{12}$ In an isothermal gas, our default case, regime (b), collisional charging, is identical (because $Z_{\text {grain }}$ depends only on $T$ for specified grain properties). Regime (c), photoelectric charging, is only different in the isothermal case if the gas is highly compressible so that fluctuations in $\rho$ are large.

In order to explore the non-linear effects of regime (c), we rerun $\mathrm{H}$ II-near L, but adopt the photoelectric scaling $t_{\mathrm{L}}^{-1} \propto Z_{\text {grain }} \propto$ $T^{1 / 2} \rho_{\mathrm{g}}^{-1}$ with the same initial homogeneous value of $Z_{\text {grain }}$ such that the constants in Table 1 are identical (i.e. only the scaling is changed). We also compare a run with $t_{\mathrm{L}}^{-1} \propto T$ corresponding to collisional charging, adopting $\gamma=5 / 3$ so that $T$ will actually vary. These H II-near L comparisons are shown in Fig. 12. It is evident that the photoelectric charging law produces dust structures with distinct topologies. The dust is more coherent, and the gas is dragged along into regions with higher dust densities and higher velocities.

\footnotetext{
${ }^{12}$ We assume throughout that we have large grains with $\left|\left\langle Z_{\text {grain }}\right\rangle\right| \gg 1$, so charge can be treated as a continuous, smooth quantity.
} 

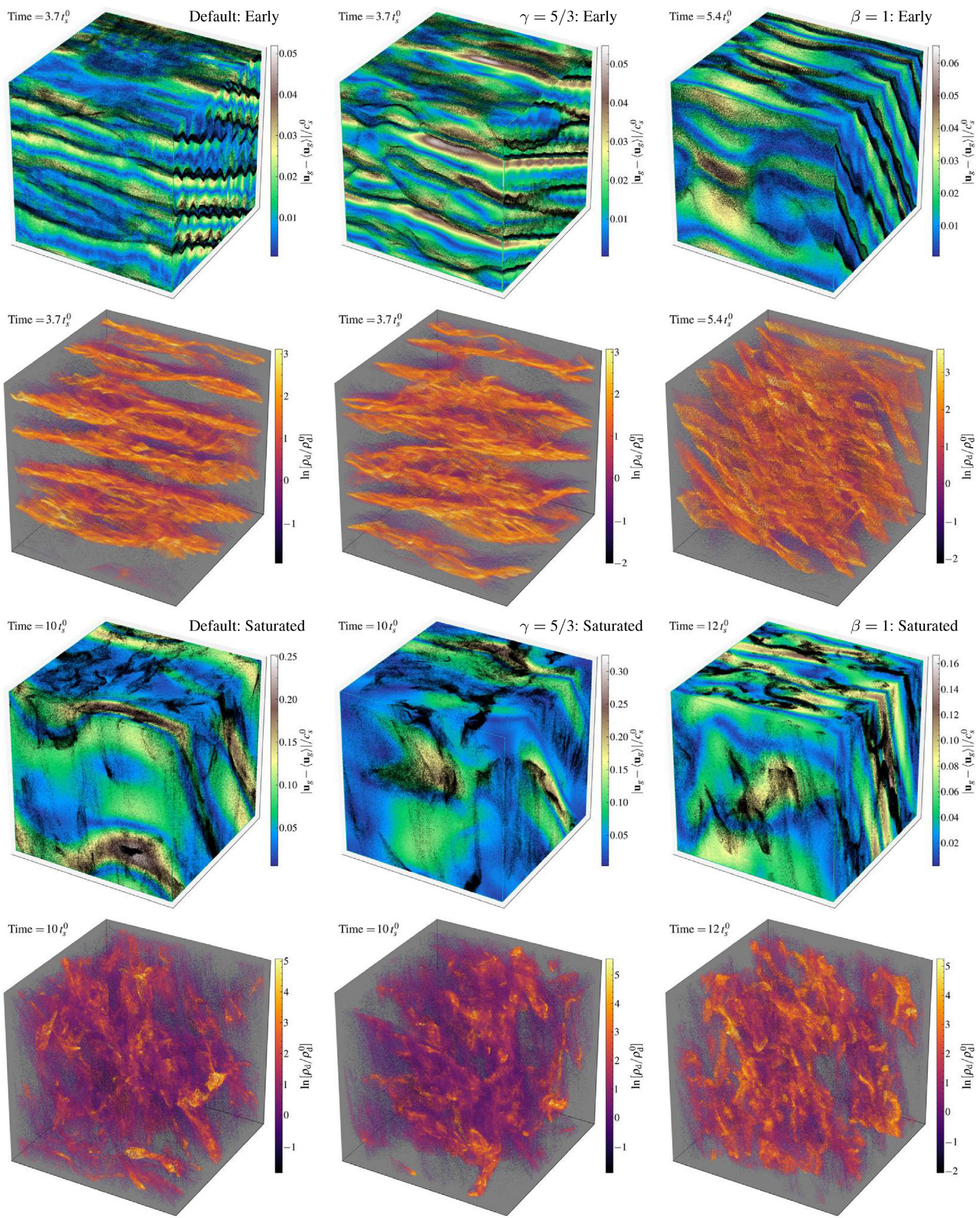

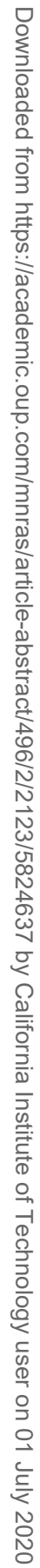

Figure 11. Dust and gas in our Example runs, as Fig. 4, but comparing physics variations instead of box sizes. We compare the 'Default' (left) run (with parameters in Table 1 and $\gamma=1$ ), to one with $\gamma=5 / 3$ (centre), and one with $\beta=1$ (right), all with otherwise equal parameters. Because the turbulence is only weakly compressible in the default $(\gamma=1)$ case, $\gamma=5 / 3$ has little effect. Shifting $\beta$ shifts the resonant mode angle, and gives slightly lower growth rates (hence the slightly later times plotted), but the behaviour is qualitatively similar. 

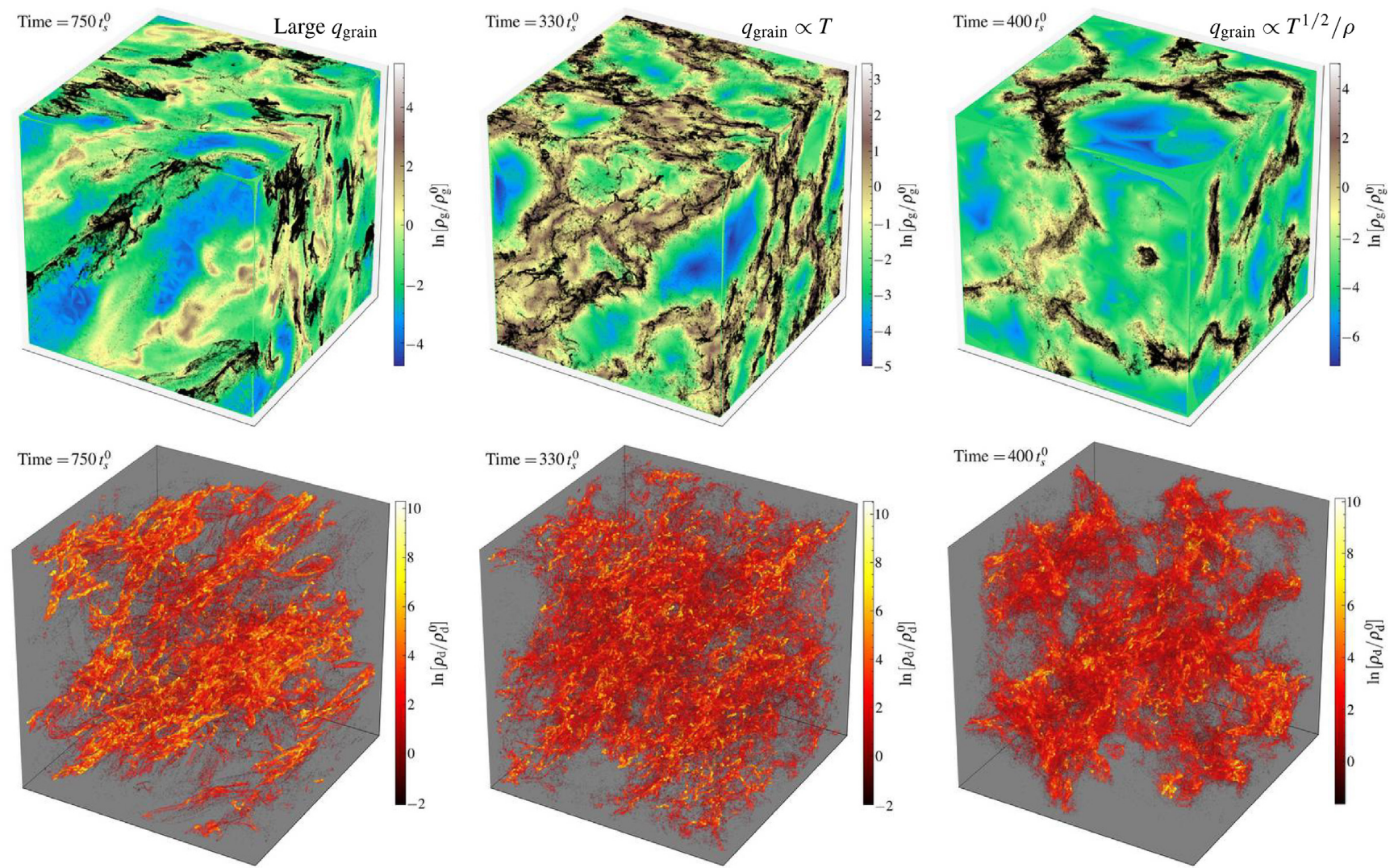

Figure 12. Comparing variations of the dust charge and charge law in the saturated turbulence of our H II-near L box (see bottom panels of Fig. 4 and Table 2). We compare (1) increasing the grain charge by an arbitrary factor $\sim 4(\gamma=1, \tau \approx 10$, constant/saturated charge; left); (2) assuming adiabatic gas (so the temperature can change) and un-saturated collisional charging $\left(\gamma=5 / 3 ; q_{\text {grain }} \propto T\right.$; middle); (3) isothermal gas assuming un-saturated photoelectric charging dominates $\left(q_{\text {grain }} \propto T^{1 / 2} / \rho ;\right.$ right $)$. The linear and early non-linear stages are very similar. Increasing charge leads to denser, more compact dust structures. In the late/saturated state, the run with the 'photoelectric' charging law produces more coherent dust structures with a distinct topology, and gas more obviously 'dragged along' to higher densities and velocities where the dust is overdense. All these runs produce stronger dust clumping in the saturated state, and develop fully non-linear structure more rapidly, than the default H II-near L simulation.

Given the (significant) physical uncertainties in predicting grain charge, we also explore the effects of re-scaling the assumed charge from our default assumptions. The results of this is shown in the left column of Fig. 12 for H II-near, the right column of Fig. 13 for CGM, and the right column of Fig. 14 for Corona. We find that increasing the charge (holding all else equal) produces faster growing, more violent instabilities.

\subsubsection{Gas equation of state}

Our default simulations adopt an isothermal equation of state $(\gamma=$ 1). This is a good approximation to many astrophysical cases of interest (e.g. the WIM, H II regions, the Solar corona), where cooling is efficient. Moreover, the majority of our runs are only weakly compressible, so changing $\gamma$ (with all other parameters fixed) only has a weak effect. In Fig. 11, we present a comparison of Example with $\gamma=5 / 3$ (with the initial pressure and all other parameters identical to the 'default' run). It is apparent that changing the equation of state in the weakly compressible limit has very little effect on the non-linear evolution of the instability. This near independence of the instabilities from changes to the equation of state also suggests that different charge scalings or drag scalings (i.e. a different dependence on $T, \rho$, etc., discussed above) will produce similar results to our default assumption (constant charge
+ Epstein drag), because gas properties do not vary dramatically regardless of $\gamma$. On smaller scales than examined here, different equation of states are more appropriate because the growth time of the instabilities may become short compared to the cooling time.

The few of our runs that are more strongly compressible in the gas all have $\left|\delta \mathbf{v}_{\mathrm{d}}\right| / c_{\mathrm{s}} \gg 1$ (as they must, since compressibility requires $\left|\delta \mathbf{u}_{\mathrm{g}}\right| / c_{\mathrm{s}} \gg 1$ and typically $\left.\left|\delta \mathbf{u}_{\mathrm{g}}\right| \lesssim\left|\delta \mathbf{v}_{\mathrm{d}}\right|\right)$. This implies that Epstein drag should better approximate dust drag forces (see Section 4.2.1). It is difficult to identify any parameter regime where Coulomb drag both dominates over Epstein drag, and has the potential to significantly alter our findings (due to its temperature or charge dependence in a non-isothermal EOS). Of our compressible simulations, we note that most represent parameters motivated by the very regions we expect to have $\gamma \approx 1$ on the scales of interest. The only case where one might expect $\gamma \neq 1$ among this set is run CGM, representative of the CGM around a bright source (where cooling is inefficient so $\gamma \approx 5 / 3$ is more appropriate). We therefore re-run this simulation with $\gamma=5 / 3$ and Epstein drag (see Fig. 13). In this case the physically realistic charge scaling is not obvious, although it is probably saturated (see Paper I). For the sake of exploring different regimes we assume unsaturated photoelectric charging, so $t_{\mathrm{L}}^{-1} \propto Z \propto T^{1 / 2} / \rho \propto \rho^{(\gamma-3) / 2}$. The resulting differences with the default parameters are quite small (cf. left-hand and middle panels in Fig. 13) 

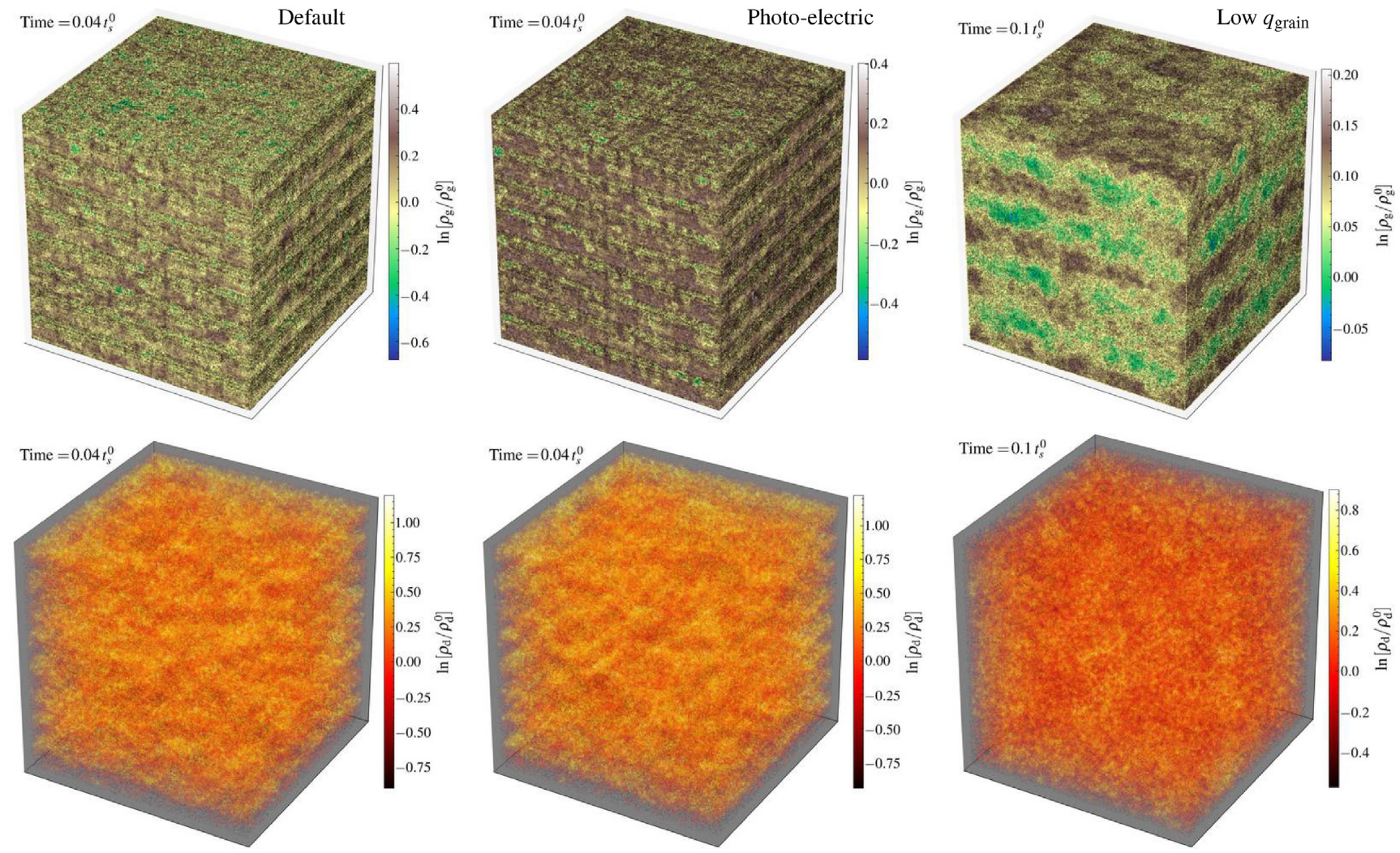

Figure 13. Comparison of CGM runs with different physics choices in the early non-linear state (as in top panels of Figs 5-8). Left-hand panels show our 'default' case ( $\gamma=1$, constant/saturated charge); middle panels show a simulation with an adiabatic EOS and the charge following the un-saturated, photoelectric-charging-dominated expectation $\left(\gamma=5 / 3, q_{\text {grain }} \propto T^{1 / 2} / \rho_{\mathrm{g}}\right)$; and right-hand panels show a case with lower grain charge $\left(q_{\text {grain }}\right.$ and $\tau 30 \times$ lower). We have also run the low- $\tau$ case with the photoelectric charge scaling, but its visual morphology is very similar (not shown). The qualitative behaviour in each case is similar, though the 'sheets' arising initially from the parallel mode are more evident in the low- $\tau$ cases since the dust (with weaker Lorentz forces) is accelerated less strongly to large $\delta \mathbf{v}_{\mathrm{d}}$. In each case the dust saturates in the 'disperse' mode with nearly isotropic $\delta \mathbf{v}_{\mathrm{d}}$ (and $\delta \mathbf{u}_{\mathrm{g}}$ ), and $\left|\delta \mathbf{v}_{\mathrm{d}}\right| \sim\left|\mathbf{w}_{\mathrm{s}}^{0}\right|$.

\subsubsection{Other parameter variations: magnetic field orientation and strength, drift geometry and speed, and dust-to-gas ratios}

Aside from these variations in drag law, gas properties, and grain charge, we consider variations of other parameters compared to the default simulations in Figs 11-19.

We examine how changes to the magnetic and drift geometries and magnitudes affect the evolution. We decrease $\beta$ by a factor of 2 (from $\beta=2$ to $\beta=1$ ) in Example, keeping all other parameters fixed. This has only minor effects on the qualitative saturation behaviour, but it does shift the resonant angles, effectively rotating the box (see the right column of Fig. 11). We compare WIM S/M/L with very low drift velocity $\left|\mathbf{w}_{\mathrm{s}}\right| / c_{\mathrm{s}} \sim 0.01(5 \times$ lower than our default case). This makes the growth rates of some modes prohibitively long, requiring too long an evolution to capture; none the less, we see many similar qualitative features emerge as in the default case, but with weaker saturated amplitudes (see Fig. 14). In Fig. 15, we re-run $\mathrm{H}$ II-near L varying the angle between $\mathbf{B}$ and $\mathbf{a}$. This does not qualitatively change the behaviour, but does shift the angle of the streams of dust in the saturated state (which tend to align with a), and produces slightly faster growth rates as a becomes more orthogonal to $\mathbf{B}$ (this is predicted by linear theory, but is not obvious, as $\mathbf{w}_{\mathrm{s}}$ actually becomes smaller).

We also investigate how changes to the dust properties affect the evolution. As shown in Seligman et al. (2019), removing dust charge in our Example box gives a linear growth rate approximately 2-3 orders of magnitude lower. We show the result of simulating this at similar times to our charged cases in Fig. 19, to show that no instability has grown. This also makes it clear that our numerical scheme does not introduce any artificial dust clumping. Finally, we simulate Example and $\mathrm{H}$ II-near with lower and higher dust-to-gas ratios $(\mu=0.001$ and $\mu=0.1)$, which are shown in Figs 16 and 18). The lower $\mu$ cases are the most interesting: as expected, the growth rate decreases by a factor $\sim 3$ (see Fig. 17) and the gas density and turbulent velocity fluctuations decrease since the forcing from the dust on the gas is weaker. Surprisingly however, lowering $\mu$ appears to enhance the strength of the saturated dust density fluctuations. This may occur because the weaker gas turbulence is less efficient at disrupting dense dust structures.

\subsection{Additional numerical tests}

In addition to the generic code validation tests described in Section 2, previous papers have tested the numerical methods here (Carballido et al. 2008; Johansen et al. 2009; Bai \& Stone 2010b; Pan et al. 2011; Hopkins 2016b, 2017; Hopkins \& Lee 2016; Hopkins \& Raives 2016; Lee et al. 2017; Su et al. 2017; Moseley et al. 2019; Seligman et al. 2019). For example, Hopkins \& Lee (2016) show that the 'finite-sampling' effects in superparticle methods, which introduce some shot noise in the particle densities and divergence between particle trajectories and gas (at the integration error level) in the perfectly coupled limit (see Price \& Federrath 2010; Ayliffe et al. 2012; Genel et al. 2013; Tricco, Price \& Laibe 2017), introduce 

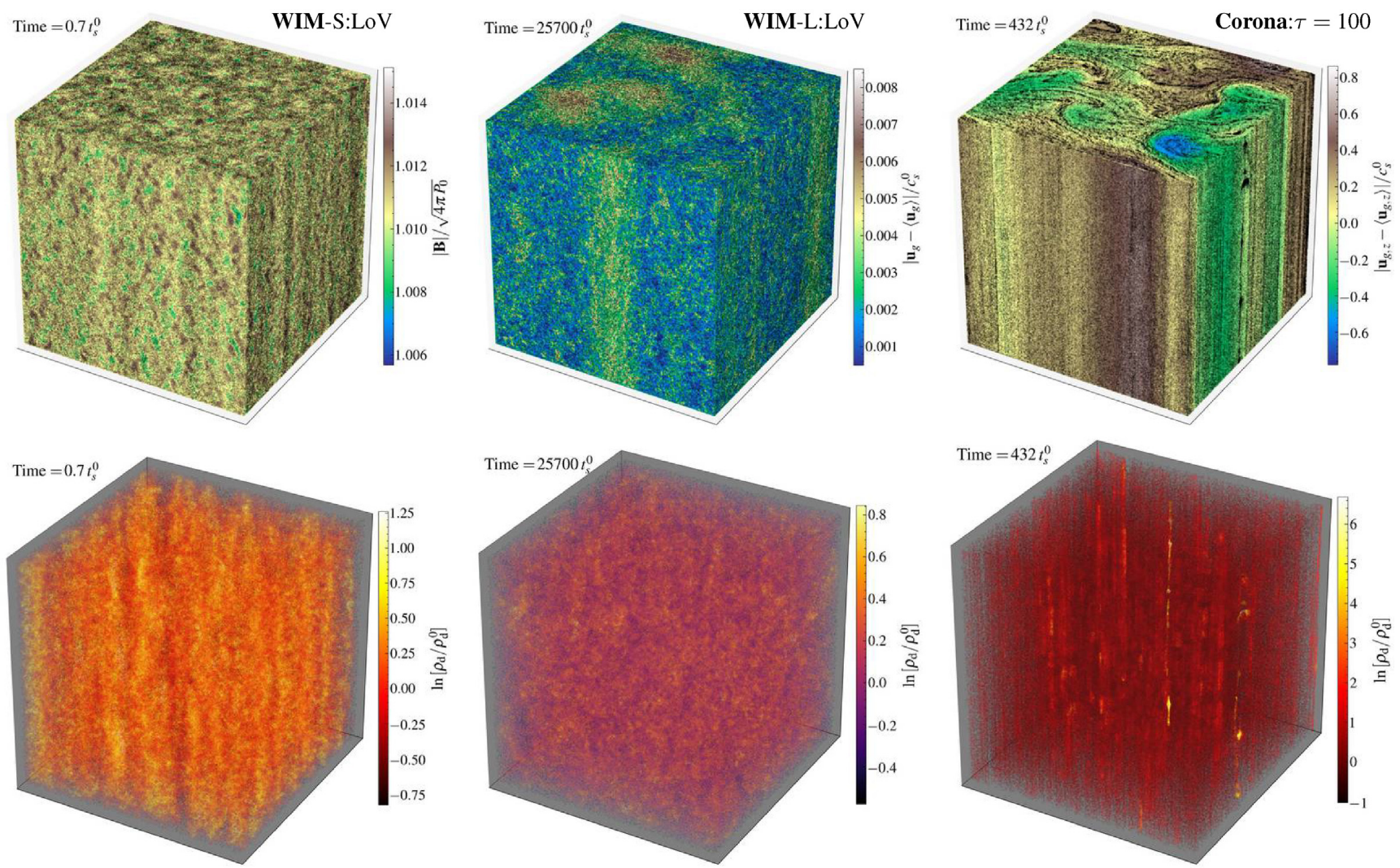

Figure 14. Left and middle: As in the bottom panels of Fig. 7, showing the WIM-S:LoV (left) and WIM-L:LoV (middle) variations at the WIM parameters in the saturated state. These variations have lower accelerations $\tilde{a}$ by a factor of 5 and, correspondingly, lower drift velocity, $\left|\mathbf{w}_{\mathrm{s}}^{0}\right| / c_{\mathrm{s}}^{0}=0.01$. The same qualitative features are evident. The linear growth rate and granular structure in box S are nearly identical in both cases. Box L exhibits similar features, with large-scale, almost laminar mixing modes, but their saturated amplitude in the gas is weaker by a factor $\sim 25$ at the same time. Note that box M has $\sim 5 \times 1$ lower growth rate and very weak turbulence, so is not shown. Right: As in Fig. 8, showing box Corona-L: $\tau=100$, which is the same as Corona-L but with $\sim 30 \times 1$ lower $q_{\text {grain }}$ and $\tau$. The $\tau$ and box size are similar to box WIM-L, and although the drift is supersonic it is significantly sub-Alfvénic. This causes the emergent morphology to qualitatively resemble WIM-L more than Corona-L. However, note the much denser 'nodes' of dust that appear.

$\sim 0.01-0.05$ dex scatter in the dust density in a supersonically turbulent medium $\left(\delta \mathbf{u}_{\mathrm{g}} / c_{\mathrm{s}} \gg 1\right)$. In both Hopkins \& Lee (2016) and Moseley et al. (2019), we show in a variety of tests that even the 'worst-case' scatter of this nature is completely negligible for our predictions, ${ }^{13}$ and that in subsonically turbulent (or laminar) media this drops to $\ll 0.01$ dex scatter. In either case this is much smaller than the saturated dispersions in $\rho_{\mathrm{d}}$ here.

As in Moseley et al. (2019) and Seligman et al. (2019), we have re-run variants of several of our standard simulations with varied numerical choices, including: (1) a different hydrodynamic solver

\footnotetext{
${ }^{13}$ We note that Tricco et al. (2017) contains an error in their comparison of 'one-fluid' dust treatments (which cannot represent multivalued dust velocity distribution functions, essential for this study; Cadiou, Dubois \& Pichon 2019; Lebreuilly, Commerçon \& Laibe 2019) to the 'tracer particle' $(\mu=0)$ method used in Hopkins \& Lee (2016). The discussion in Tricco et al. (2017) incorrectly compares to simulations in Hopkins \& Lee (2016) which adopted different dust parameters $(\tilde{\alpha})$, a different dust drag law, and did not use the same weighting for the PDFs/dispersions measured. If one correctly compares the simulations from Hopkins \& Lee (2016) which have similar $\tilde{\alpha}=0.001-0.01$ to the cases studied in Tricco et al. (2017), and weights the PDFs identically, then the agreement is reasonably good, and in fact the simulations in Hopkins \& Lee (2016) predict slightly smaller fluctuations in the dust-to-gas ratio $\rho_{\mathrm{d}} / \rho_{\mathrm{g}}$ than those in Tricco et al. (2017), the opposite of their conclusion.
}

(for Example, WIM L, H II-far S/M/L), we use the 'meshless finite mass' (MFM) method from Hopkins (2015); (2) a different scheme for calculating gradients and reconstruction of the magnetic field at cell faces (for Example, Corona S), specifically the 'constrained gradient' MHD scheme in Hopkins (2016b); (3) using a naive (nonmanifestly energy-conserving) explicit leapfrog integrator instead of the Boris integrator for the Lorentz forces on grains (in Example, H II-near S/M/L, Corona S/M/L, WIM M/L, CGM); and (4) using different initial conditions, namely glass-like instead of lattice initial particle configurations (for Example). Choices (2), and (4) have no significant effect on any results we explore. We find that choice (1), the MFM method, moderately suppresses the initial maximum growth rates of the modes in the box, owing to its slightly less accurate integration of highly subsonic flows near the grid scale (effectively, the fastest growing resolved modes in the simulation correspond to those at $\sim 4-6$ times the grid scale, instead of $\sim 2-3$ times the grid scale). Choice (3) has negligible effects on boxes Example and H II-near S/M/L, where the dust is strongly clumped and $\tau$ is not extremely large. However, for boxes Corona, WIM, and CGM, where $\tau \geq 100$ is larger and the systems often end up in the 'disperse' mode, we find that it becomes important to use an integrator (like our default Boris scheme) that manifestly conserves Lorentz orbits. Otherwise, the dust tends to drift and spiral outwards and gain energy artificially, an effect that is well-known in standard particle-in-cell codes. 

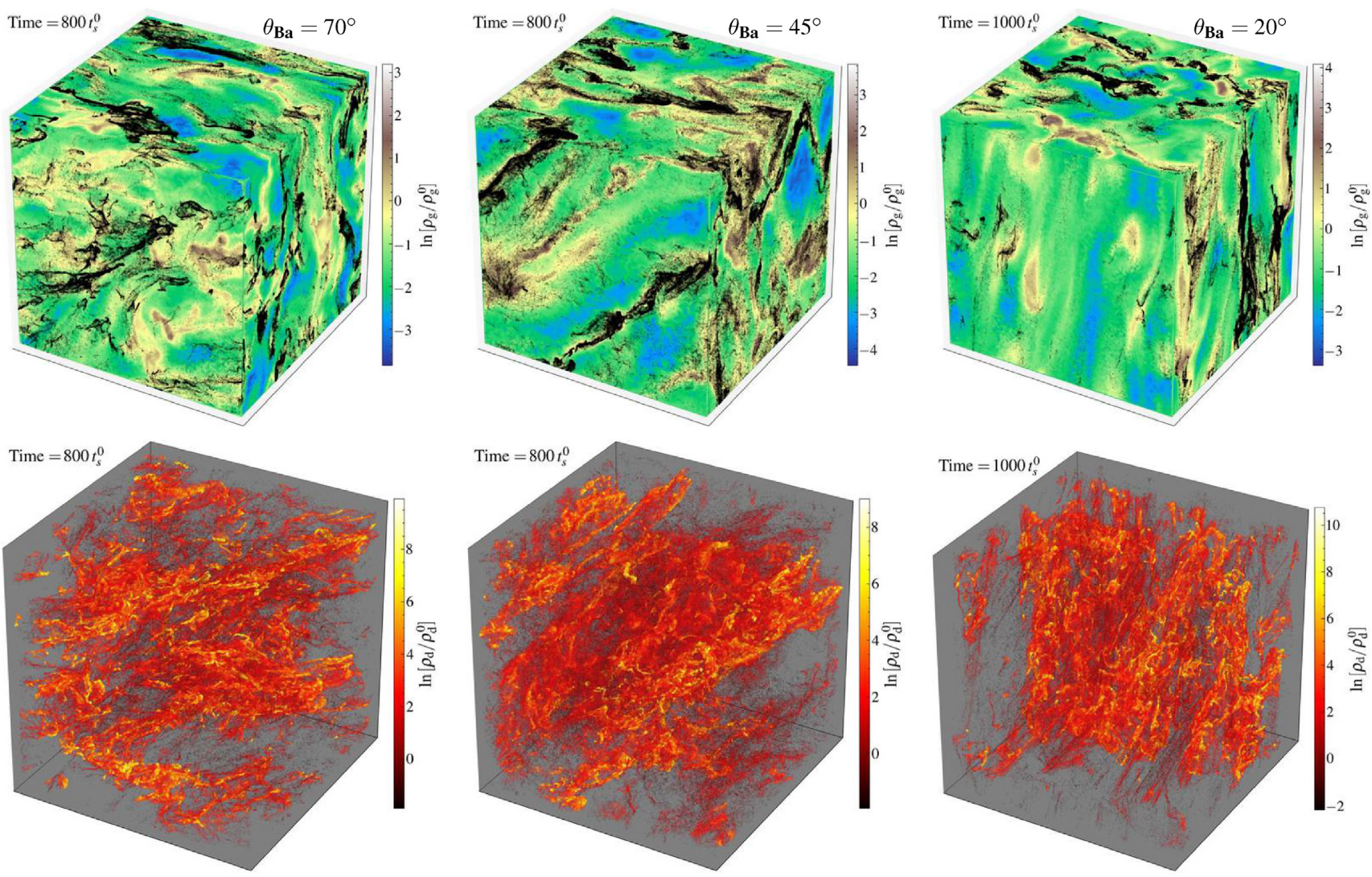

Figure 15. As in bottom panels of Fig. 4, comparing variations of the acceleration direction in the H II-near L box in the saturated state. We take our default run (shown in the middle panels) and, keeping all other parameters fixed, vary the direction of the external net acceleration between dust and gas (a) so $\theta_{\mathbf{B a}}=$ $\left(70^{\circ}, 45^{\circ}, 20^{\circ}\right)$ at $\left(l e f t\right.$, middle, right). The case with a closer to parallel $\mathbf{B}_{0}$ (right) produces slightly lower initial growth rates, so grows more slowly, even though the equilibrium drift velocity $\left|\mathbf{w}_{\mathrm{s}}\right| c_{\mathrm{s}}$ is slightly larger. However, it saturates with slightly stronger fluctuations. The case with a closer to perpendicular $\mathbf{B}$ grows slightly faster (despite smaller $\left|\mathbf{w}_{\mathrm{s}}\right| / c_{\mathrm{s}}$ ). The qualitative structure is none the less broadly similar in each case.

We have also run a number of resolution tests. Our default runs here use $2 \times 128^{3}$ elements $\left(128^{3}\right.$ each in gas and dust). All of our runs here have also been run at lower resolution $\left(2 \times 32^{3}\right.$ and $\left.2 \times 64^{3}\right)$, and one at higher $\left(2 \times 256^{3}\right)$. Fig. 20 compares the saturated state in this resolution series. The qualitative behaviour is similar across this range of resolutions. However, without some sort of physical isotropic viscosity, or non-fluid effects (e.g. the ion gyro radii), these instabilities are linearly unstable at all wavelengths, with growth rates that increase with $k$. Thus, increasing resolution and keeping all else fixed will always resolve new, faster growing modes at smaller scales, so it is not possible to undertake a formal convergence analysis. A more formal resolution study, as well as a number of additional numerical validation tests and more extensive discussion, are presented in Moseley et al. (2019). ${ }^{14}$ For example, fig. B3 in Moseley et al. (2019) compares the PDFs of the same properties studied here (e.g. dust-to-gas ratio) as a function of the relative number of dust and gas elements (varying $N_{\text {dust }} / N_{\text {gas }}$ by a factor of 64) - as expected, the resulting PDFs are essentially

\footnotetext{
${ }^{14}$ Note that in Moseley et al. (2019), where we studied the pure acoustic RDI, we noted that the high- $k$ modes (with $\left|\mathbf{w}_{\mathrm{s}}\right| \gg c_{\mathrm{s}}$ ) were difficult to resolve in some cases because the growth rates become sharply peaked around a narrow resonant angle of width $\delta \theta \propto \mu^{2 / 3} k^{-1 / 3}\left|\mathbf{w}_{s}\right|^{-1}$. As shown in Paper I, the MHD modes have a much more complex but also broader resonant structure, especially at the lower $\mathbf{w}_{\mathrm{s}}$ and higher $\tau$ values studied here. This contributes to making our results significantly less sensitive to resolution.
}

identical up e.g. Poisson sampling in the tails of the distribution. We have verified this with our runs here as well. As shown in Hopkins \& Lee (2016), Lee et al. (2017), and Moseley et al. (2019), these PDFs are also robust to the details of kernel density estimators for dust or gas.

The saturated amplitudes of the velocity and density fluctuations are relatively insensitive to resolution in run Example. This suggests that most of the power is dominated by the well-resolved largest modes in the box, rather than the fastest growing but smallest scale modes. This is consistent with our power spectrum analysis in Seligman et al. (2019), where we showed explicitly in run Example that most of the power in the saturated state was in modes a factor $\sim 2$ around the box scale. This may not be true in some other runs, e.g. H II-far L, where the dust clumps on small scales.

\subsection{Distribution functions}

Figs 21-27 examine the PDFs of fluctuations for the seven simulations considered in this paper. We see that the large diversity of behaviour in morphology, saturated amplitude, and anisotropy is mirrored in the diverse array of PDF shapes. The PDFs are often highly non-Gaussian; this comes (broadly speaking) in two 'forms'.

First, some PDFs exhibit substantial substructure, e.g. AGB$\mathrm{M}$ or $\mathrm{H}$ II-far in $\delta \mathbf{u}_{\mathrm{g}}$ or $\delta \mathbf{B}$, which have multiple bumps (sharp inflection points/changes in curvature) or even second peaks. This is directly related to coherent, large-scale morphological structures in 

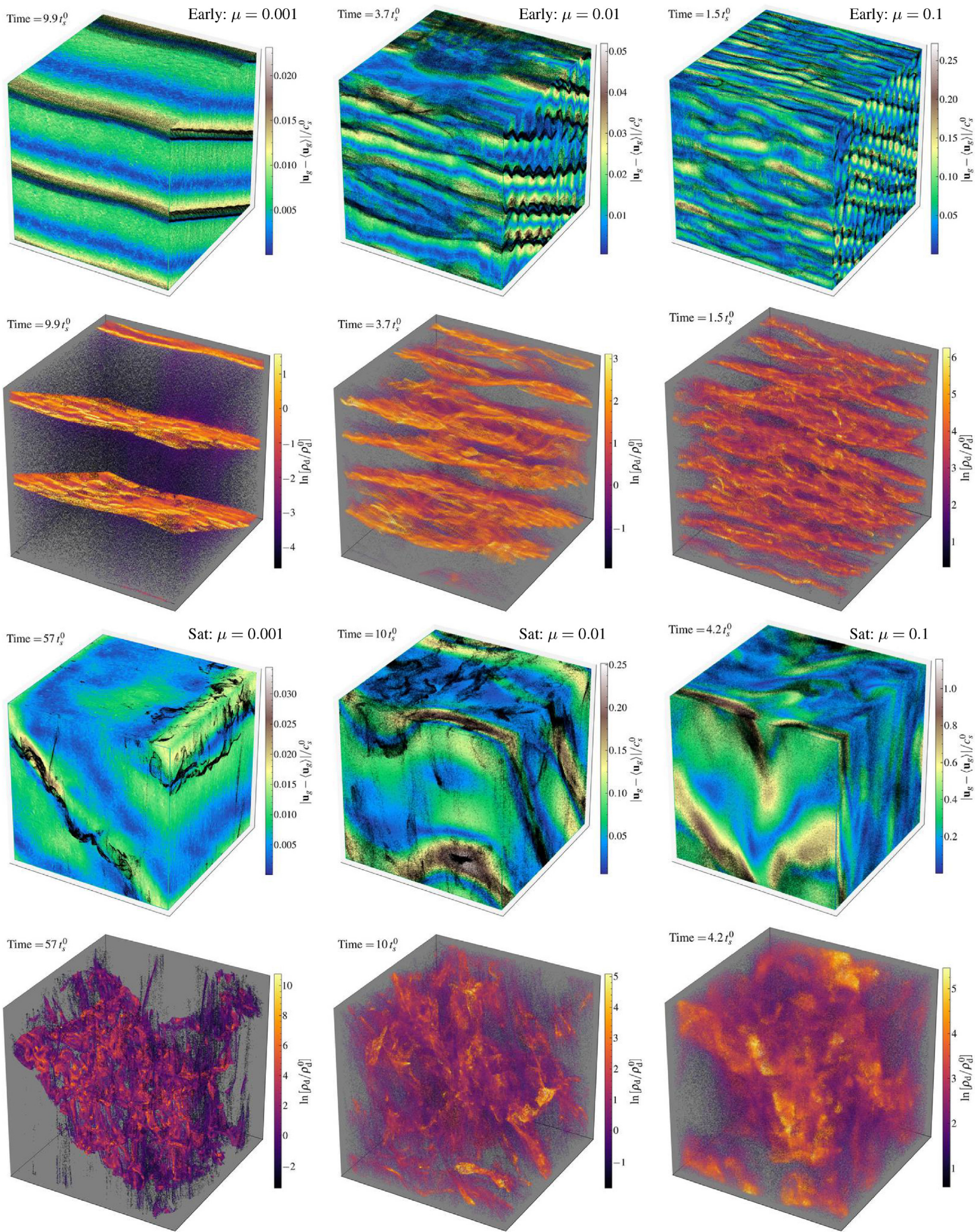

Figure 16. Like Fig. 11, comparing our Example run with three different dust-to-gas ratios $\mu=0.001$ (left), 0.01 (our default; middle), 0.1 (right). The broad form of the instabilities is similar in each case, and the growth time-scale scales as $\sim \mu^{1 / 2}$, as expected from linear theory. At early times the wavenumber (number of 'sheets') corresponding to the most visually obvious mode is lower for lower $\mu$ : this corresponds to the fastest-growing wavelength where the 'aligned' mode dominates in Fig. 17, so again is predicted by linear theory. The saturated gas turbulence is stronger with increasing $\mu$, as expected because the forcing from dust is stronger). However, the low- $\mu$ case exhibits extremely small, dense sheets and filaments that do not turn into less dense or 'fuzzy' patches at late times, as occurs in the high- $\mu$ case. In fact most, in each run, of the dust mass resides at approximately the same dust-to-gas ratio (i.e. the typical 'overdensity' is systematically larger at lower $\mu$ ), and the highest dust-to-gas ratios appear in the lowest $\mu$ box. 

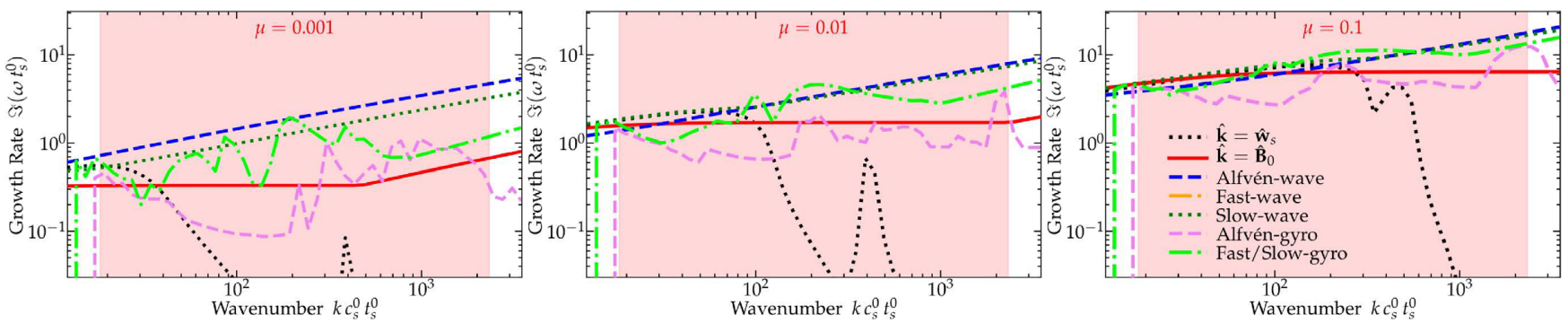

Figure 17. Linear growth rates, as in Fig. 2, for the variants of Example with varied dust-to-gas ratio $\mu$ in Fig. 16 (as labelled). The growth rate normalization scales approximately as $\sim \mu^{1 / 2}$, explaining the relative time-scales in Fig. 16. The wavenumber of the 'sheets' that dominate the early non-linear phase corresponds to the fastest growing (highest) wavenumbers $k$ where the aligned modes (with $\hat{\mathbf{k}}=\hat{\mathbf{B}}$ or $\hat{\mathbf{k}}=\hat{\mathbf{w}}_{s}$; red and dotted black lines) have growth rates comparable to or greater than the other modes. The MHD-wave (Alfvén and slow) RDIs generate the 'corrugation' of the sheets - the oscillations with $\hat{\mathbf{k}}$ nearly perpendicular to the 'aligned' modes of the sheets.
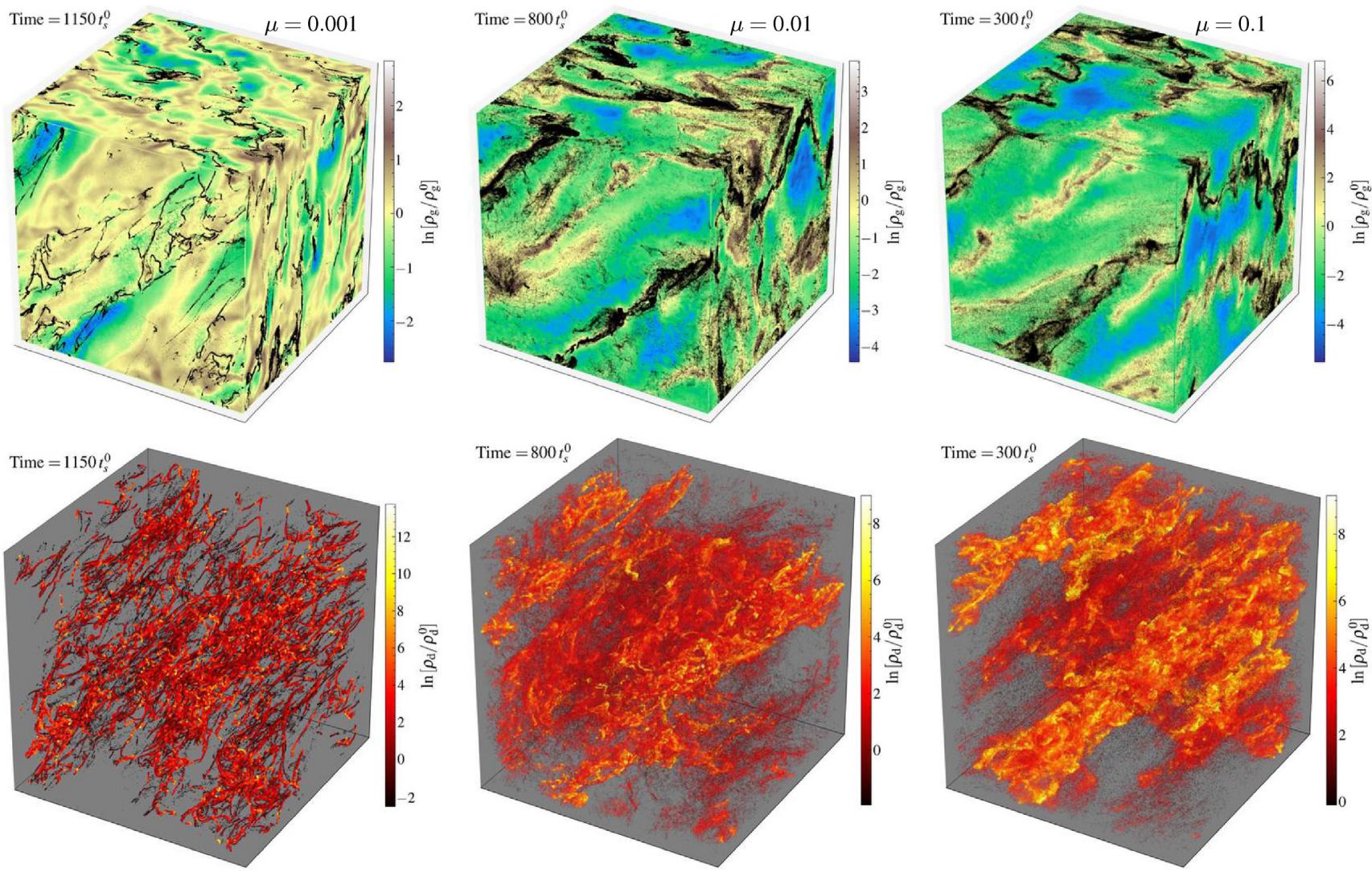

Figure 18. Like Fig. 16 (bottom panels), comparing variants of the H II-near L run with three different dust-to-gas ratios $\mu=0.001$ (left), 0.01 (default; middle), 0.1 (right) in the saturated state. The conclusions are similar to Fig. 16: the growth times and wavenumbers scale with predictions of linear theory as $\mu^{1 / 3 \rightarrow 1 / 2}$ and the gas turbulence is stronger at high- $\mu$, but the low- $\mu$ case retains extremely dense filaments or clumps that do not get 'broken up' or 'spread out' at later times. The dust reaches extremely high overdensities in the low- $\mu$ case.

Figs 4-7. Each such 'peak' corresponds to 'patches' (subvolumes of the parent box) which have either much larger or smaller dust density within them, and have essentially de-coupled from one another, evolving non-linearly almost independently.

Second, most of the 'smooth' PDFs have non-Gaussian tails, which are most commonly exponential or 'stretched exponential' in form: i.e.

$$
P(q) \propto \exp \left(-\left|q / q_{0}\right|^{\gamma}\right)
$$

for some $q$ and $\gamma$. For example, the $\delta$ B PDFs in almost all cases have this form, as do the $\delta \mathbf{u}_{\mathrm{g}}$ PDFs in Corona and WIM S/M and H IIfar S/M. PDFs with exponential or stretched-exponential tails are common in certain types of gas turbulence, velocity distributions of granular gases, and passive scalar concentrations in subsonic incompressible turbulence (Ruiz-Chavarria, Baudet \& Ciliberto 1996; Yakhot 1997; Ben-Naim \& Krapivsky 2000; Antal, Droz \& Lipowski 2002; Ernst \& Brito 2002; Kohlstedt et al. 2005; Aranson \& Tsimring 2006; Monchaux, Bourgoin \& Cartellier 2010; Hopkins 2013b; Colbrook et al. 2017). This generically arises from a competition between driving and dissipation. Consider the distribution of velocities in a statistically homogeneous system (so $P\left(\mathbf{u}_{g}, \mathbf{x}\right)=P\left(\mathbf{u}_{g}\right)$ ), where the velocities are driven by an uncorrelated stochastic process with the specific energy injection rate $\sim \dot{u}_{0}\left(v / v_{*}\right)^{\xi}$ (the effective 'diffusion coefficient' in velocity 

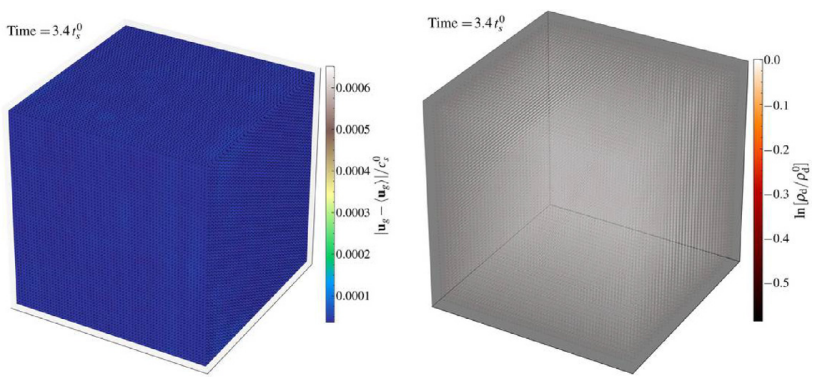

Figure 19. Visualizations for a variation of the Example run, removing all grain charge (Lorentz forces on grains). As shown in Seligman et al. (2019), the linear growth rate becomes multiple orders of magnitude smaller in this limit. We see here that at similar times to those when the default Example run has gone strongly non-linear (Fig. 16), no structure has developed.
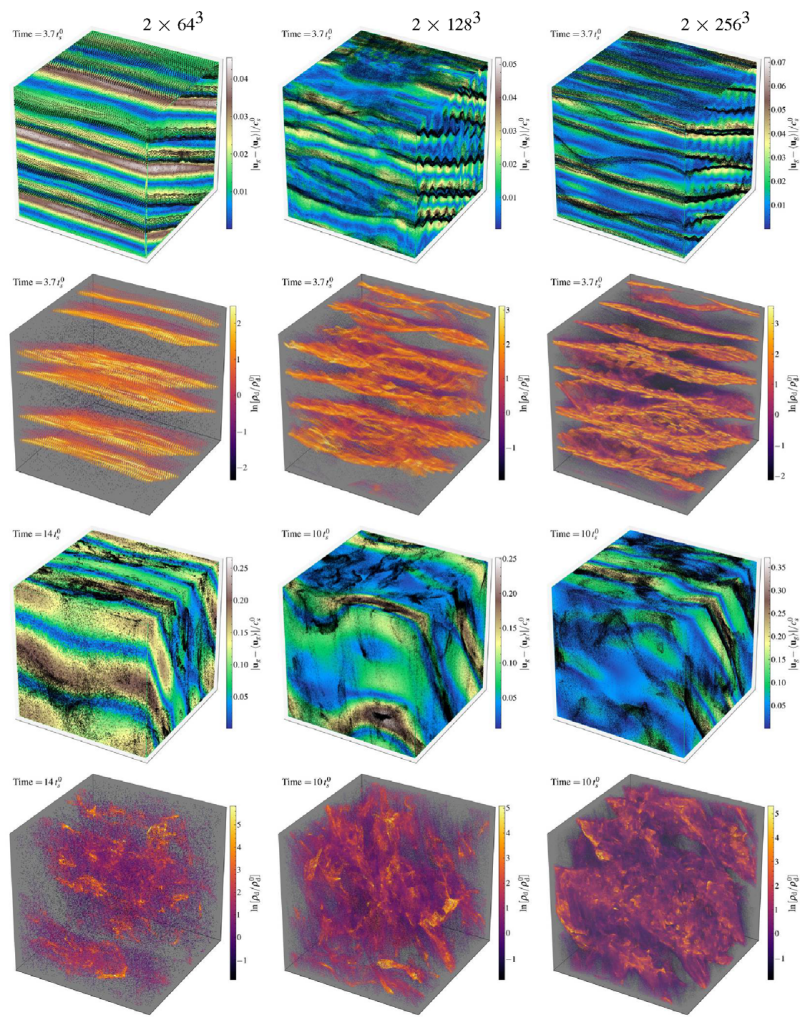

Figure 20. Comparison of the Example run at different resolution levels: $2 \times 64^{3}$ (left), $2 \times 128^{3}$ (our 'default' case; centre), and $2 \times 256^{3}$ (right). The instabilities develop slightly more quickly at increasing resolution, owing to the fact that the growth rates increase almost monotonically with $k$ (Fig. 2). As expected, we can also see more detailed, finer, and sometimes denser structure in the dust at higher resolution (the gas remains relatively smooth). However, the qualitative form of the instabilities and the saturated amplitude of the gas turbulence remains similar.

space), and damped ${ }^{15}$ with rate $t_{\text {damp }}^{-1}\left(v / v_{*}\right)^{\psi}$. In steady state, if these driving and damping processes dominate, the PDF obeys

$\dot{u}_{0}\left(v / v_{*}\right)^{\xi} \partial^{2} P(v) / \partial v^{2} \sim-t_{\text {damp }}^{-1}\left(v / v_{*}\right)^{\psi} P(v)$ for each component, the solutions of which obey $P(v) \propto$ $\exp \left(-\left|v / v_{*}\right|^{\gamma}\right)$ at large $v$ with $^{16}$

$\gamma=1+(\psi-\xi) / 2, \quad v_{*} \sim \sqrt{\dot{u}_{0} t_{0}}$.

So, for the simple case of white noise (Brownian) driving and constant damping, or any case where driving and damping depend on $v$ in the same manner $(\psi \approx \xi)$, the tails are exponential. The characteristic width of the PDF is simply given by $v_{*}^{2} \sim \dot{u}_{0} t_{0}$; i.e. the energy injected in a damping/dissipation time.

While it is easy to qualitatively understand the range of PDF tails in this manner, such arguments fall far short of a predictive model. In other words, it is not possible (given the arguments here alone) to predict the PDFs and structure functions purely from the various simulation parameters. For example, it is not a priori obvious what the relevant driving and damping rates should be in the saturated regime. If turbulence dominates both the non-linear forcing and the damping (e.g. eddies shearing apart growing modes, as we argue sets the saturation amplitude of some modes below) then both injection and damping times might scale with eddy turnover times, as in e.g. the standard theory of supersonic turbulent density fluctuations (Vazquez-Semadeni 1994; Hopkins 2013a,b; Squire \& Hopkins 2017). But driving could also arise directly from mode growth, or from non-linear parasitic modes, while damping could also stem from drag (acting on dust velocity fluctuations) or sound waves (for gas pressure fluctuations) - the dominant terms do not have to be the same for each type of fluctuation.

Some of the PDFs exhibit strong asymmetries, with a much stronger 'tail' in one direction (e.g. $\mathbf{u}_{\mathrm{g}}$ and $\mathbf{v}_{\mathrm{d}}$ in AGB L/XL, $\rho_{\mathrm{d}}$ in $\mathrm{H}$ II-far M/L or CGM). In cases like AGB or H II-far where the tails extend to both larger $\mathbf{u}_{g}$ in the direction of acceleration $\mathbf{a}$ and larger $\rho_{\mathrm{d}}$, this relates to the fact that regions with non-linearly larger local $\rho_{\mathrm{d}} / \rho_{\mathrm{g}}$ experience faster local growth of the instabilities, and more efficient acceleration of the coupled dust-gas mixture (because the gas acceleration scales as $\sim \mu \mathbf{a})$. Cases with a large tail in $\rho_{\mathrm{d}}$ towards smaller $\rho_{\mathrm{d}}$ (CGM, WIM) likely arise because dust is locally expelled from small pockets that are local vorticity maxima. This is essentially the generic mechanism studied in Hopkins (2016a) and is well-known in terrestrial particulate 'preferential concentration' studies (Squires \& Eaton 1991; Fessler, Kulick \& Eaton 1994; Rouson \& Eaton 2001; Gualtieri, Picano \& Casciola 2009; Monchaux et al. 2010). Essentially, grains are centrifugally 'flung out' of high-vorticity regions, and concentration is a side effect as grains collect in-between. Finally, there are examples that are highly non-Gaussian but do not neatly fit into any of the classes above (e.g. $\mathbf{v}_{\mathrm{d}}$ in CGM or WIM L).

We also compare the volume-weighted $\left(P_{V}\right.$, the probability that a given random volume has some value) and mass-weighted (dust weighted by dust mass, gas by gas mass; $P_{M}$ ) statistics. If the density variations are small, these must be similar. Indeed for the gas velocity and magnetic field $\left(\mathbf{u}_{\mathrm{g}}\right.$ and $\left.\mathbf{B}\right)$ the two statistics rarely differ dramatically, as the gas density fluctuations are small. The few cases (e.g. H II-near L) where some differences appear are those with the most dramatic gas density fluctuations, and they still do not differ qualitatively. Even for the dust, where $\rho_{\mathrm{d}}$ varies strongly, the differences are rarely dramatic in $\mathbf{v}_{\mathrm{d}}$ (an exception is H II-near $\mathrm{L}$, where dust in the high- $\rho_{\mathrm{d}}$ filaments is coherently moving relative to the low- $\rho_{d}$ 'background', giving rise to larger dispersions when volume-weighted).

\footnotetext{
${ }^{15}$ Note this damping corresponds to any process damping the fluctuations, not necessarily the dust drag.
}

${ }^{16}$ For $\psi-\xi<-2$, the solutions become peaked functions $P(v)$ which asymptote to constant $P>0$ as $v \rightarrow \infty$. 


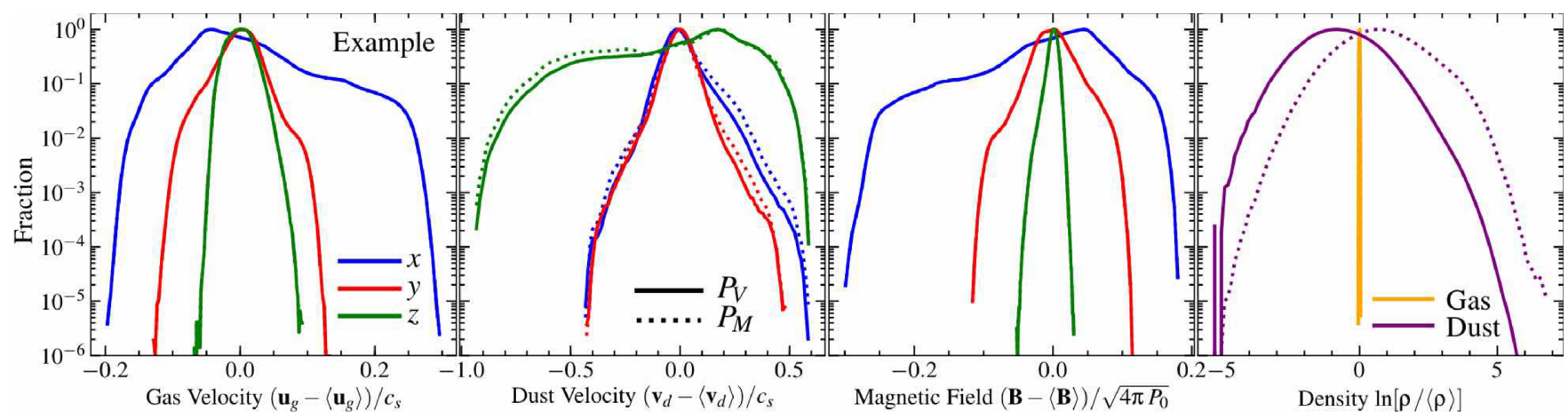

Figure 21. PDFs of gas and dust velocities, and magnetic field fluctuations, in each direction $x y z$ (labelled), as well as the dust and gas densities. This is for the Example run, averaging over the last 10 snapshots (in saturation). For each PDF, solid lines show the volume-weighted PDF $\left(P_{V}\right)$, while dotted lines show the mass-weighted PDF $\left(P_{M}\right)$. PDFs are normalized to the same peak amplitude for convenience. Substantial structure is evident, related to coherent large-scale morphological structures in Fig. 11. The PDF tails often exhibit highly non-Gaussian statistics.

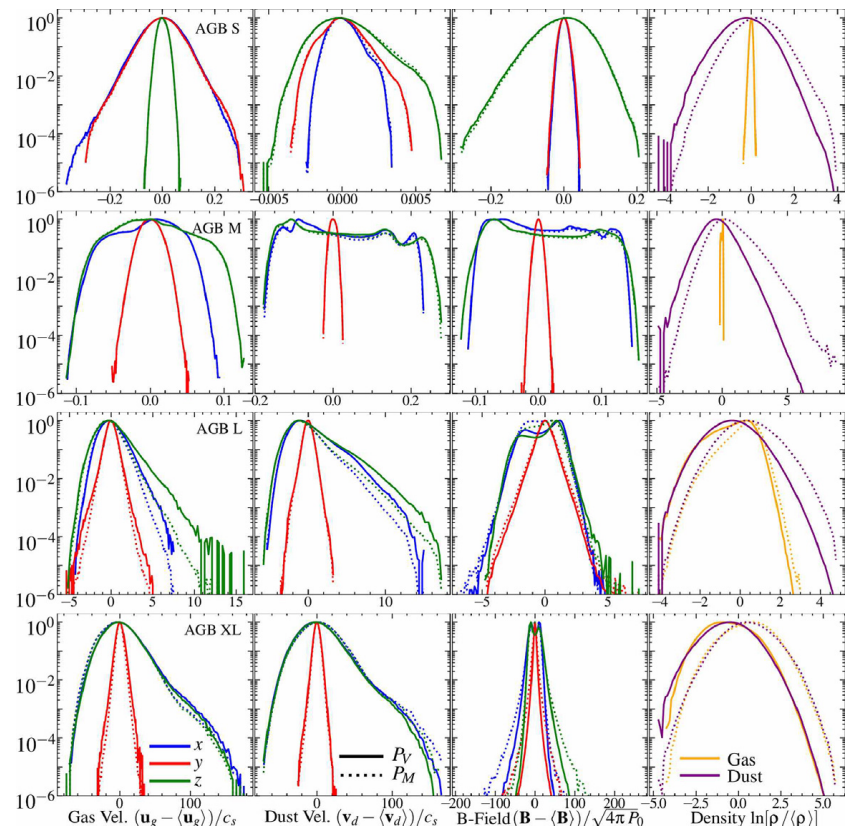

Figure 22. PDFs for AGB S/M/L/XL from top-to-bottom, as Fig. 21. All cases exhibit complicated structure in $\mathbf{u}_{\mathrm{g}}, \mathbf{v}_{\mathrm{d}}, \mathbf{B}$. Anisotropy is obvious: statistics here are similar in the direction of acceleration $(\hat{x}-\hat{z})$ and fluctuations are weaker in the perpendicular $(\hat{y})$ direction, except for the smallest box, which reverses this. The velocity and density PDFs typically exhibit exponential tails $\left(P \propto \exp \left(-\left|v / v_{*}\right|\right)\right)$, discussed in the text. Dust density fluctuations are much larger than gas, except on the largest scales. Most of the dust mass lies in regions with above-average dust density, while most of the volume has lower-than-average dust density.

In the density PDFs, volume and mass weighting makes more difference. Some of this is by definition: because $\rho=\mathrm{dMass} / \mathrm{dVolume}$,

$P_{M} \propto \rho P_{V}$

if both are measured in differentially small regions. So $P_{M}(\rho)$ will always be biased to higher $\rho$ than $P_{V}(\rho)$. Let us define the mass and volume-weighted averages $\langle(\cdot)\rangle_{M}$ and $\langle(\cdot)\rangle_{V}$ respectively, the volume-weighted variance $S$ in the $\log$-density field $\chi \equiv \ln \rho$, and the usual 'clumping factor' $C$ :

$S \equiv S_{V} \equiv\left\langle\ln \left[\rho / \rho_{0}\right]^{2}\right\rangle_{V}-\left\langle\ln \left[\rho / \rho_{0}\right]\right\rangle_{V}^{2}=\left(\delta \ln \left[\rho / \rho_{0}\right]\right)^{2}$

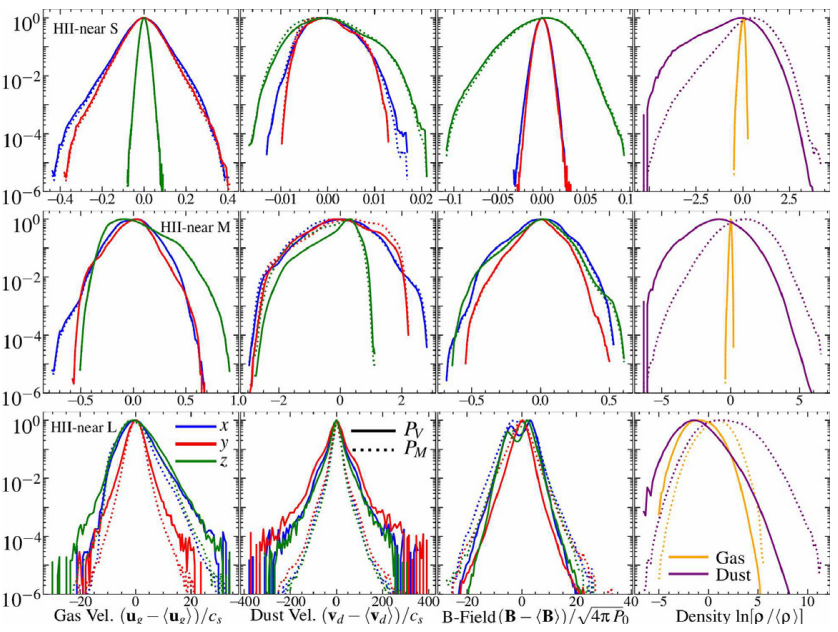

Figure 23. PDFs for $\mathrm{H}$ II-near $\mathrm{S} / \mathrm{M} / \mathrm{L}$ from top-to-bottom, as Fig. 22. Substructure and anisotropy are common again. Some PDFs are close to Gaussian. Others (e.g. B in box L) are almost pure exponential. The $\rho_{\mathrm{d}}$ PDFs are highly skew, with large fluctuations so mass versus volume weighting makes a large difference. The 'clumping factor' $C=\left\langle\rho_{d}^{2}\right\rangle /\left\langle\rho_{d}\right\rangle^{2} \sim(3,6,100)$ in boxes (S, M, L). In box L, $\mathbf{v}_{\mathrm{d}}$ exhibits a narrow 'core' with a broad or 'wide' stretched-exponential distribution superposed (the PDF has 'fat tails'). These are all suggestive of strong intermittency (see Section 4.4).

$S_{M} \equiv\left\langle\ln \left[\rho / \rho_{0}\right]^{2}\right\rangle_{M}-\left\langle\ln \left[\rho / \rho_{0}\right]\right\rangle_{M}^{2}$

$\rho_{0} \equiv\langle\rho\rangle_{V}$

$C \equiv \frac{\left\langle\rho^{2}\right\rangle_{V}}{\langle\rho\rangle_{V}^{2}}$

where $\rho_{0}=\langle\rho\rangle_{V}$ follows from our definitions. If $P_{V}(\rho)$ is exactly lognormal, then mass-conservation implies: (1) the volumeweighted mean/median/mode of $\chi$ is $\left\langle\ln \left(\rho / \rho_{0}\right)\right\rangle_{V}=-S_{V} / 2$, (2) $P_{M}$ is also lognormal with $S_{M}=S_{V}$ and mass-weighted $\left\langle\ln \left(\rho / \rho_{0}\right)\right\rangle_{M}=$ $+S_{V} / 2$, (3) $C=\exp \left(S_{V}\right)$.

One way that we can quantify the deviations from lognormal in these PDFs is via the extent to which $S_{M} \neq S_{V}$ or $C \neq \exp \left(S_{V}\right)$ (this is commonly used as a diagnostic in supersonic turbulence studies). This can be read off from the values of $S_{V}=\left(\delta \ln \left[\rho / \rho_{0}\right]\right)^{2}$ and $C$ in Table 2. In several cases (e.g. AGB) the results are consistent with lognormality $\left(C \approx \exp \left(S_{V}\right)\right)$. But in some very strongly clumped 


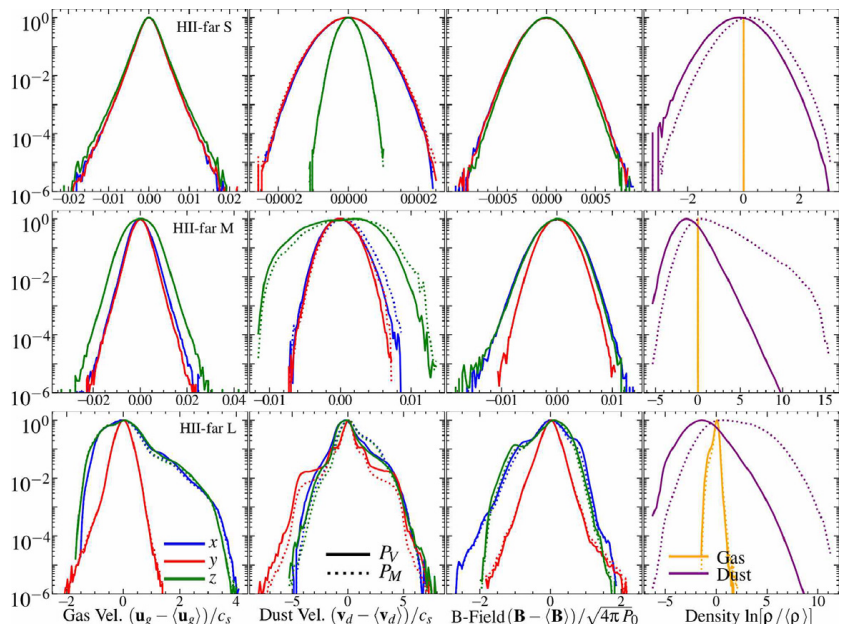

Figure 24. $\mathrm{PDF}$ for $\mathrm{H}$ II-far $\mathrm{S} / \mathrm{M} / \mathrm{L}$ from top-to-bottom, as Fig. 22 Again note substructure and 'fat tails' in the PDFs (especially in box L, related to the large-scale coherent filaments in Fig. 5); nearly exponential PDFs for $\mathbf{u}_{g}$ in boxes $\mathrm{S}, \mathrm{M}$; and large skew tails to high $\rho_{d}$. While the gas is very weakly compressible here, the dust exhibits enormous and non-Gaussian density fluctuations: consider box M, where $\left|\delta \mathbf{u}_{g}\right| / c_{s} \sim$ $\left|\delta \mathbf{v}_{d}\right| / c_{s} \sim|\delta \mathbf{B}| / \sqrt{4 \pi P_{0}} \sim\left|\delta \ln \rho_{g}\right| \sim 0.01$, but the dust $\delta \ln \rho_{\mathrm{d}} \sim 1.3$ (2.5) weighted by volume (mass). Moreover since the dust PDF is highly nonGaussian, $\sim 0.1$ per cent of the dust mass lies at densities $\rho_{d} \gtrsim 10^{7}\left\langle\rho_{d}\right\rangle$

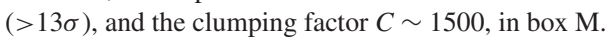

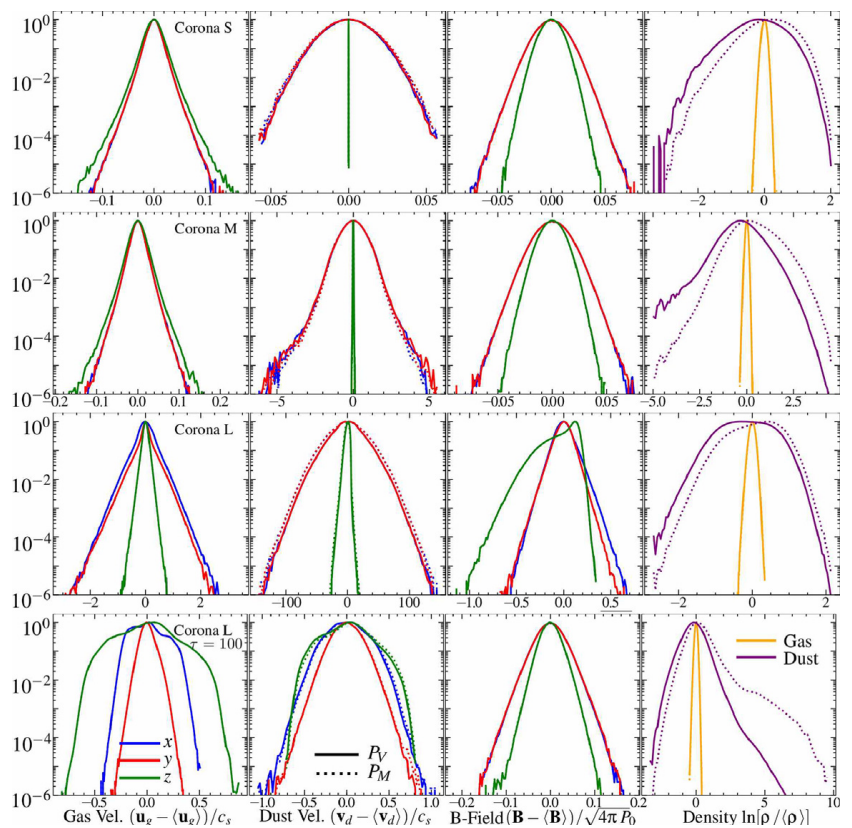

Figure 25. PDFs for Corona S/M/L (top/second/third), as Fig. 22 (bottom shows run $\mathrm{L}: \tau=100$ ). The 'default' runs all exhibit weak $\rho_{\mathrm{g}}$, and nearly pure exponential $\mathbf{u}_{\mathrm{g}}$ fluctuations, with large $\mathbf{v}_{\mathrm{d}}$ fluctuations in the plane perpendicular to $\hat{\mathbf{B}}$ but small along $\hat{\mathbf{B}}$. The asymmetry in $\delta \mathbf{B}_{z}$ in box L stems from the large energetic cost of such fluctuations at low- $\beta$. The low- $\tau=$ 100 run exhibits qualitatively different behaviour: the fluctuations in $\mathbf{v}_{\mathrm{d}}$ are $\sim 100 \times$ smaller (and $\delta \mathbf{u}_{\mathrm{g}}, \delta \mathbf{B}$ are $\sim 10 \times$ smaller), however while the 'core' of the $\rho_{\mathrm{d}}$ PDF has similar width it exhibits a 'tail' (dominated by the caustics in the folded dust 'sheets' in Fig. 14) to orders-of-magnitude larger $\rho_{\mathrm{d}}$.
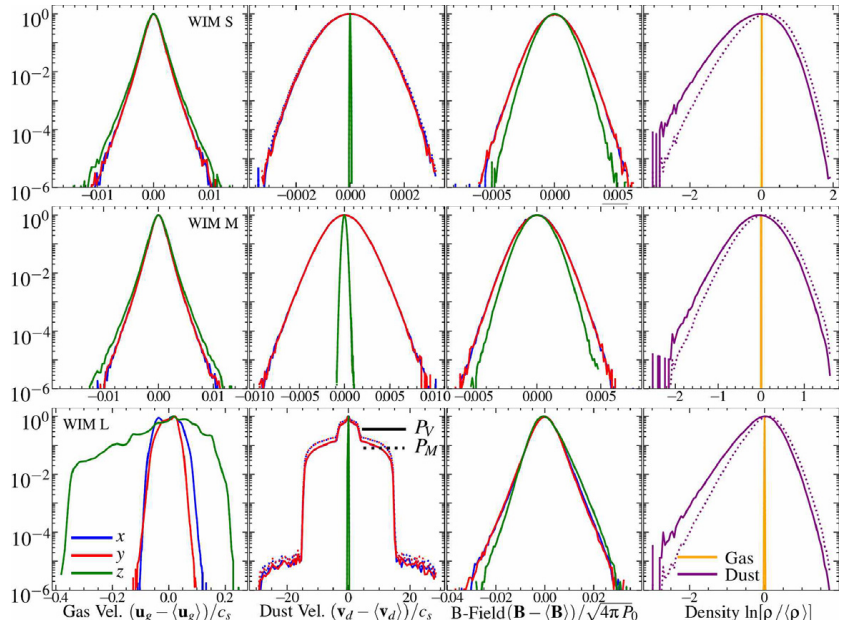

Figure 26. PDFs for WIM S/M/L from top-to-bottom, as Fig. 22 Anisotropy is similar to Corona, and gas is very weakly compressible. $\rho_{\mathrm{d}}$ fluctuations are primarily to low- $\rho_{\mathrm{d}}$ (dust expelled from some regions), so e.g. the clumping factor $C \sim(1.2,1.02,1.1)$ (biased to high $\rho_{\mathrm{d}}$ ) is small. In box $\mathrm{L}$, note the $\mathbf{v}_{\mathrm{d}}$ PDFs, symmetric perpendicular to $\mathbf{B}$, with progressively broader flat-topped PDFs 'superposed' to produce large tails.

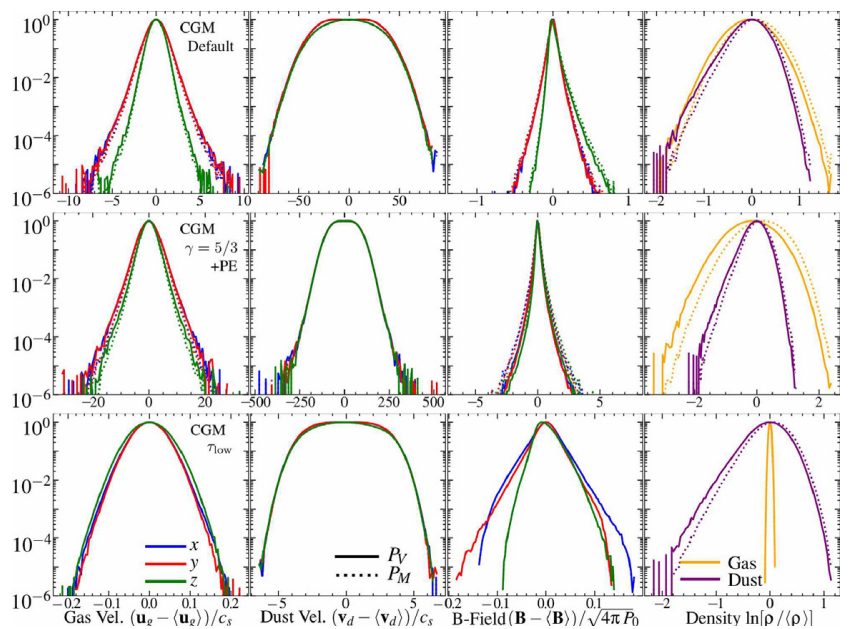

Figure 27. PDFs for CGM, as Fig. 22, in our 'Default' run (top), run with $\gamma=5 / 3$ and photoelectric grain charge scaling (middle), and lowcharge (' $\tau_{\text {low }}$ ' i.e. $\tau \sim 5000$; bottom) run. Lower $\tau$ (weaker Lorentz forces) produces weaker dust velocity dispersions, sourcing weaker gas motions. The photoelectric grain charging produces stronger dust acceleration. Fluctuations in all cases are close to isotropic. While $\mathbf{u}_{\mathrm{g}}$ and $\mathbf{B}$ PDFs are subGaussian (exponential in $\mathbf{B}$ ), the PDFs for $\mathbf{v}_{\mathrm{d}}$ are super-Gaussian (flat-topped with rapid falloffs).

cases we have, for the dust, $S_{M}>S_{V}$ and $C \gg \exp \left(S_{V}\right)$ (e.g. H II-near and H II-far M,L have $S_{V} \sim 2$ and $S_{M} \sim 4-6$ ). Specifically, given the measured $\delta \ln \left(\rho_{d} /\left\langle\rho_{d}\right\rangle\right)=S_{V}^{1 / 2}$ (for the dust), and assuming lognormality $\left(C=\exp \left(S_{V}\right)\right)$ would predict $C \sim 5-10$ in these cases. In contrast the measured $C$ is $C \sim 100-300$ in H II-near L and $\mathrm{H}$ II-far $\mathrm{L}$, and $C \sim 1500$ in $\mathrm{HII}-\mathrm{far} \mathrm{M}$. Conversely, the PDFs with tails towards low $\rho_{\mathrm{d}}$ have smaller $C$ than would be predicted from $\delta \ln \left(\rho_{\mathrm{d}} /\left\langle\rho_{\mathrm{d}}\right\rangle\right)$; e.g. the CGM and WIM runs have $C \sim 1.0-1.1$. This owes directly to the large, asymmetric tails in $\rho_{\mathrm{d}}$ visible in Figs 21-27.

Another, perhaps simpler, way of emphasizing this is to note that the most extreme cases (e.g. H II-far M, or H II-near L and Example 
with lower $\mu=0.001$ ) have extreme tails, where $\gtrsim 0.1$ per cent of the dust mass lies at densities $\gtrsim 10^{7}$ times larger than the mean (reaching as high as $\sim 10^{9}-10^{12}$ ). This corresponds to values $\gtrsim$ 15 standard deviations from the volume-weighted median of the density PDF.

\section{DISCUSSION}

From the analysis in Section 4, we can identify a number of important conclusions about both the linear and early non-linear phases, as well as the saturated and late non-linear phases.

\subsection{Linear and early growth phases}

(i) In all cases, the instabilities grow and produce strongly nonlinear properties in the dust (see Figs 4-13). This is not surprising, since all cases here are linearly unstable (Fig. 2). However, it does show that non-linear growth can occur even when the growth time-scale is shorter than the dust 'stopping time' or Larmor time (i.e. $\Im\left(\omega t_{s}\right)>1$ or $\Im\left(\omega t_{s}\right)>\tau$, in Fig. 2). This implies that the instabilities grow faster than the time-scale for the system to reach a 'new' equilibria, which is not a trivial conclusion.

(ii) Generally, the instabilities exhibit the most rapid initial growth at the smallest scales. All wavelengths here are linearly unstable, with growth rates generally increasing at smaller wavelengths (Fig. 2), because we do not include explicit dissipation (e.g. viscosity). ${ }^{17}$ Thus, within each parameter set, the smaller boxes evolve more rapidly in fixed physical units (e.g. $t_{s}^{0}$ ). Moreover within each box, in most cases we see small-scale modes saturate first, with the scale of structures increasing until box-scale modes saturate (compare e.g. early and later times in Figs 4, 5, and 11).

(iii) The simulations broadly reproduce the expected growth rates from linear theory in the early linear growth phase, as depicted in Fig. 9. We demonstrate and discuss this in more detail for a few representative acoustic RDIs in Moseley et al. (2019, fig. 2 therein) and our Example case in Seligman et al. (2019, figs 4 and 5). For modes that are unstable on all scales (e.g. the Alfvén MHDwave RDI), the early growth rate appears to match the linear theory prediction for wavenumbers $k \sim(1-3) / \Delta x_{0}$ (where $\Delta x_{0}=L_{\mathrm{box}} / N_{1 \mathrm{D}}$ is the initial inter-element grid spacing; again see also Moseley et al. 2019, Fig. 2). This suggests that the growth rate can be recovered even if the full wavelengths are resolved by just $\sim 2-6$ interparticle spacings. ${ }^{18}$ At later times, the box-scale modes take over as the strongest growing modes, until saturation.

(iv) The presence of magnetic fields and grain charge, along with associated Lorentz forces, play a critical role in the linear development of the instability. For several cases studied here, if we artificially remove the dust charge (Lorentz forces), or magnetic fields, the instabilities become stable or orders-of-magnitude more slowly growing. Fig. 19 shows that no structure develops at late times in the Example run with zero grain charge. Moreover, increasing the grain charge $\left(q_{\text {grain }}\right.$ and $\tau$ ) produces faster growing instabilities with more violent saturated dust behaviour, in both the

\footnotetext{
${ }^{17}$ In fact (see Paper I) some of instabilities (e.g. the Alfvén wave and gyro resonances) have growth rates that can continue to rise with decreasing wavelength even below the field-parallel viscous scale, potentially down to the ion gyro radii or even further.

${ }^{18}$ For rigorous demonstration of this in idealized test problems, as well as formal convergence studies, see Moseley et al. (2019), appendices A and B. This is also consistent with the results using different codes and numerical methods, e.g. Johansen \& Youdin (2007).
}

'clumped' and 'dispersed' regimes (see Figs 12 and 13). This is despite the fact that Lorentz forces decrease the 'equilibrium' dust drift velocities, and magnetic fields increase the pressure support of the gas, which would naively appear to be 'stabilizing' effects. But these terms also introduce a variety of new dust and gas modes (e.g. Alfvén and slow waves, dust gyro motion) which in turn dramatically increase the number of accessible 'resonances' for the instabilities (as well as introducing new energy sources for the instabilities).

(v) The 'resonances' where linear growth rates are maximized are sufficiently vigorous that they can often be identified well into the non-linear evolution. These maximal resonances occur where the 'natural frequency' of advection $\mathbf{w}_{\mathrm{s}} \cdot \mathbf{k}$ or gyro motion $t_{L}^{-1}$ in the dust matches the 'natural frequency' of an Alfvén or magnetosonic wave in the gas. Even in the non-linear phases of evolution, these resonances manifest as particular angles or wavelengths of the structures that form, as can be seen in Figs 16 and 17.

These results are all, to some extent, predicted from the linear theory in Paper I, but we both verify the linear theory results and confirm that these conclusions persist even well into non-linear evolution.

\subsection{Saturation and late non-linear phases: generic conclusions}

As noted above, the saturated states exhibit some qualitatively different behaviours, but there are some generic conclusions that apply to all of our runs. We discuss these first, before exploring the physics that is distinct in different saturated states.

(i) All of the systems saturate in a turbulent quasi-steady state. This is evident in Fig. 9, where the velocity dispersion has clearly reached saturation, although in some cases certain fluctuations continue to grow very slowly. The finite velocity dispersion of the dust grains in the saturated state does not shut down fluctuations, even in the cases that reach a nearly isotropic grain velocity distribution function (e.g. Fig. 27). In other words, the turbulence reaches a saturated steady state, rather than the instability quenching itself.

(ii) The predicted structure here is qualitatively completely different from that formed in 'passive' dust experiments, in which the dust moves as a tracer particle in externally driven turbulence (neglecting the forces from dust on gas, i.e. the momentumconserving 'backreaction' terms). In other words, 'passive' dust cannot generate these instabilities or structures - indeed, the RDIs do not exist with 'passive' dust. Some of the most noticeable differences are found in the large-scale dust morphology; there is a much stronger prevalence of filaments and sheets here, compared to no strong anisotropy present in 'passive dust' simulations. Also in 'passive dust' simulations, the PDFs of dust density do not have the same shape or qualitative scalings as those presented here (compare Hopkins \& Lee 2016; Lee et al. 2017, or the discussion in Moseley et al. 2019). In most cases we study, the dust density fluctuations are vastly larger in our simulations (with 'active' dust which can drive the RDIs) as compared to an analogous 'passive-dust' case. For example, with $\tilde{\alpha} \sim 0.001$, similar to some of our most strongly unstable and dust-clumped cases in H II-near, H II-far, and AGB L, Lee et al. 2017 typically found $\delta \ln \left(\rho_{\mathrm{d}} / \rho_{\mathrm{g}}\right) \sim 0.01$ for 'passive' grains (orders-of-magnitude smaller than our result here). Perhaps most importantly, the scaling of the strength of dust clustering with $\tilde{\alpha}\left(L_{\text {box }}\right)$ or $\tilde{\phi}(\tau)$ in 'passive dust' studies is, in many cases, almost opposite those here (e.g. Lee et al. 2017 found fluctuations in dustto-gas ratio with 'passive' dust were only strong at $\tilde{\alpha} \gg 1$ ). 


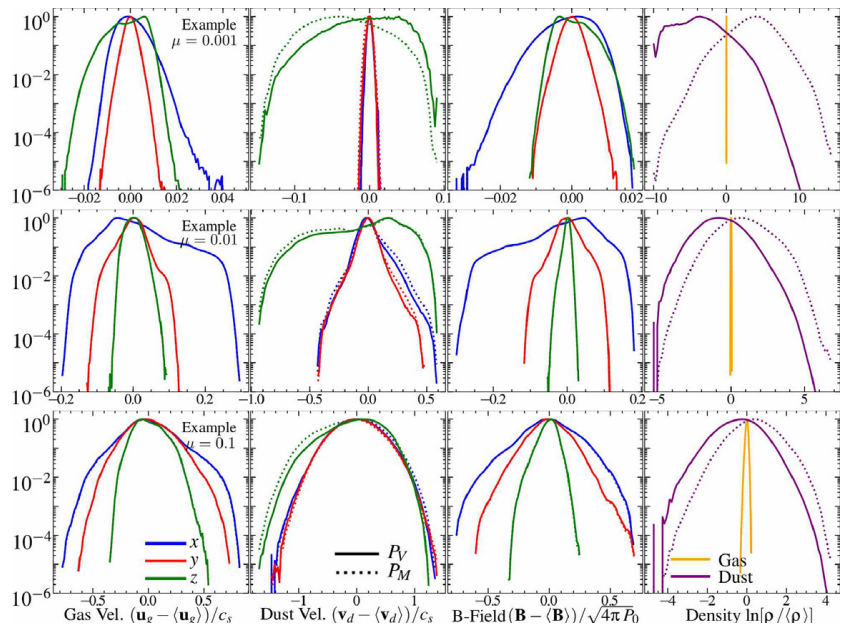

Figure 28. PDFs for the runs in Fig. 16, where we vary the dust-to-gas ratio $\mu=0.001$ (top), $\mu=0.01$ (default; middle), $\mu=0.1$ (bottom) for run Example. The PDFs for gas fluctuations and dust velocity become broader, more Gaussian, and more isotropic at higher $\mu$, consistent with greater mixing and more uniform driving. But (per Fig. 16), the dust density fluctuations are larger at lower $\mu$.

(iii) Details of the gas equation-of-state, the functional form of the drag law (Coulomb+Epstein or just Epstein), or the grain charge scaling (dependence on local temperature and density) do not qualitatively alter our conclusions, although they certainly have quantitative effects (Table 2, and Figs 11, 12, and 13.). Larger dust charge generally produces more violent saturation (Figs 12 and 13). The non-linear behaviour of the instabilities does not depend sensitively on particular alignments or anti-alignments between acceleration and magnetic field directions (except in so far as the resonant angles change; e.g. Fig. 15), and in fact, cases where the two are more strongly anti-aligned can even grow faster, despite weaker grain drift. Likewise, modest variation in parameters like the equilibrium grain drift velocity (relative dust-gas acceleration) or magnetic $\beta$ do not qualitatively alter the behaviour or character of saturation (Figs 11 and 14). Lowering the dust-to-gas ratio produces slower initial growth and weaker gas turbulence, as expected. However, surprisingly, it can produce non-linear clustering in the dust that is as strong, or even stronger, than higher $\mu$ cases (Figs 16, 18, 28, and 29).

(iv) Most systems are driven towards approximate equipartition between gas velocity and magnetic field fluctuations. This agreement - i.e.

$\left\langle\rho_{g}\right\rangle \delta \mathbf{u}_{g}^{2} / 2 \sim \delta \mathbf{B}^{2} / 8 \pi$

is at the order-of-magnitude level, as shown in Fig. $10 .{ }^{19}$ This result is independent of the initial $\beta$ (from $\beta_{0} \sim 0.001$ to 1000). In some cases this involves strong amplification of $\mathbf{B}$ fields (e.g. from $\beta \sim$ 1 to $\sim 0.01$ in AGB-XL). Because of this, while the instabilities

\footnotetext{
${ }^{19}$ There are a few notable exceptions with $\left\langle\rho_{g}\right\rangle \delta \mathbf{u}_{g}^{2} / 2 \gg \delta \mathbf{B}^{2} / 8 \pi$. Two are WIM-L and Corona-L: $\tau=100$, although the variant WIM-L:LoV ('low drift velocity') and 'default' (higher- $\tau$ ) Corona-L have $\left\langle\rho_{g}\right\rangle \delta \mathbf{u}_{g}^{2} / 2 \approx \delta \mathbf{B}^{2} / 8 \pi$ to within $\sim 10$ per cent. In both of these exceptions, the gas moves nearly incompressibly and two-dimensionally, so the $\mathbf{B}$ fields are moved in the $x y$ plane but not compressed, generating negligible B fluctuations (see Fig. 7). Our default (high- $\tau$ ) CGM boxes also exhibit low $\delta \mathbf{B}$, though here it may be because the fluctuations are dominated by small- $k$, random gas motions which do not cause an effective coherent dynamo.
}

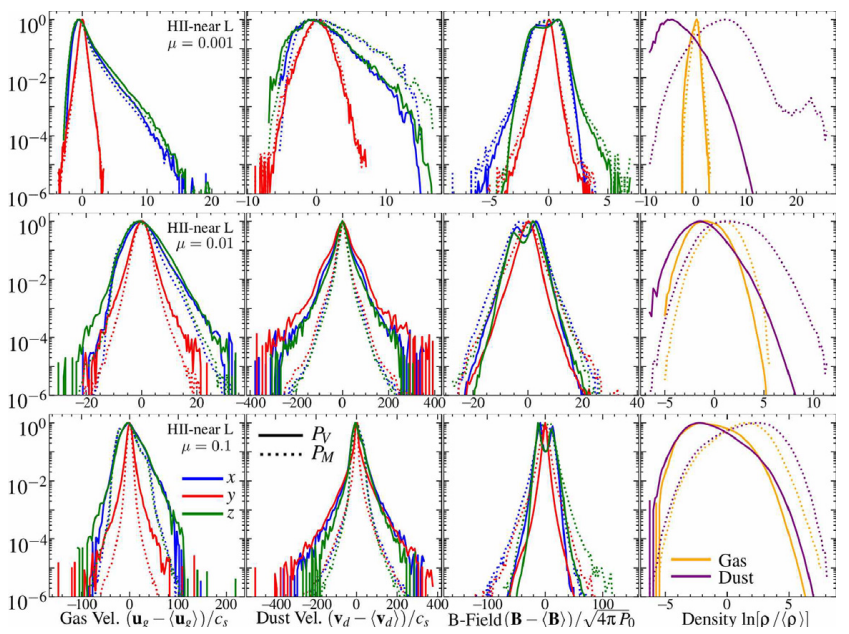

Figure 29. As Fig. 28, comparing different dust-to-gas ratios $\mu=0.001$ (top), $\mu=0.01$ (default; middle), $\mu=0.1$ (bottom) for run $\mathrm{H}$ II-near L (in Fig. 18). Conclusions are similar to Fig. 28. Note the extreme fluctuations in the low- $\mu$ case: most of the volume has $\rho_{d}<0.01\left\langle\rho_{d}\right\rangle$ (i.e. is highly dust-depleted), while most of the dust mass resides in structures with $\rho_{d} \gtrsim$ $400\left\langle\rho_{d}\right\rangle$ (highly dust-enriched), and $\gtrsim 0.1$ per cent of the dust mass is at $\rho_{d} \gtrsim 10^{9}\left\langle\rho_{d}\right\rangle$ with the highest value here reaching $\sim 10^{12}\left\langle\rho_{d}\right\rangle$.

can drive highly supersonic turbulence in some cases, it is usually trans-Alfvénic.

(v) All of the instabilities examined saturate with sustained gas turbulence. To rough order-of-magnitude, saturation often occurs when the eddy turnover time-scale on the box scale becomes shorter than the box-scale linear growth time-scale i.e. $\left|\delta \mathbf{u}_{g}\right| / L_{\mathrm{box}}$ $\sim \Im\left(\omega\left[L_{\text {box }}\right]\right)$. However, as discussed below, for both very small boxes and some magnetically dominated boxes, other criteria (e.g. equipartition between magnetic tension and driving by dust) may instead set the saturation amplitude (see Fig. 10). In any of these cases, the strength of the saturated gas turbulence increases with dust-to-gas ratio and box size/wavelength (see Fig. 10, Table 2). This can be understood physically, since the forcing of the dust on to the gas becomes stronger relative to pressure and magnetic forces. Provided some gas velocity fluctuations, the gas density fluctuations roughly follow the usual relation for pure isothermal MHD turbulence,

$\left[\delta \ln \left(\rho_{g} /\left\langle\rho_{g}\right\rangle\right)\right]^{2}=\ln \left[1+\left(b\left|\delta \mathbf{u}_{g}\right| / c_{s}\right)^{2}\right]$,

but with substantial variation in the 'compressibility' $b \sim 0.2-1$ (see Fig. 10). B can be related to $\mathbf{u}_{g}$ as described above. Anisotropy in the gas properties can usually be understood as a direct reflection of the anisotropy in the fastest-growing linear modes at the box scale (see below).

(vi) The dust saturation is ubiquitously more complex than the gas saturation. In some cases, the dust exhibits extremely strong 'clumping' or clustering, with a wide range of distinct morphologies and topologies (e.g. differently connected sheets, filaments, or clumps). In the most extreme cases simulated here, the dust overdensities reach magnitudes of $\sim 10^{9-12}$ times the mean, as seen in Figs 21-29! In other cases, the dust is 'dispersed' throughout the box, with nearly isotropic, large, velocity dispersions. Qualitatively, the anisotropy of $\mathbf{v}_{d}$ and relation between $\mathbf{v}_{d}$ and $\mathbf{u}_{g}$ reflect those of the fastest-growing linear modes at the box scale (see below). Generically, on 'intermediate' and 'large' scales $k \lesssim 1 /\left(\mu c_{s} t_{s}\right)$, we expect and see $\left|\delta \mathbf{v}_{d}\right| \gtrsim\left|\delta \mathbf{u}_{g}\right|$, while on 'small' scales $k \gtrsim 1 /\left(\mu c_{s} t_{s}\right)$, 
$\left|\delta \mathbf{v}_{d}\right| \lesssim\left|\delta \mathbf{u}_{g}\right|$ (see Fig. 10). ${ }^{20}$ On the very largest scales, the dust density fluctuations $\delta \rho_{d}$ are comparable to gas density fluctuations $\delta \rho_{g}$. But, while $\delta \rho_{g}$ decreases with scale, $\delta \rho_{d}$ does not, because there is no internal pressure resisting compressions. In fact, some of the most extreme dust-density fluctuations appear when the gas is nearly incompressible, and they actually become stronger at lower dust-to-gas ratios (Figs 28-29).

(vii) The statistics of both dust and gas fluctuations are often highly non-Gaussian (Figs 21-29), with exponential or 'stretched exponential' tails and, in some cases, coherent substructure. This is generally associated with strong intermittency and stochastic driving in dissipative systems (Section 4.4). These strong deviations from Gaussianity mean, for example, that the mass-weighted dust density fluctuations can deviate substantially from volumeweighted fluctuations, and in some cases a significant fraction of the dust mass ( $>0.1$ per cent) can reside at values $\gtrsim 15-20$ standard deviations from the median.

\subsection{Saturation: 'clumped' states}

Although it is clear here that the saturated states are diverse and occupy a continuum of properties, we attempt to classify them into two very broad 'regimes', based on their morphology and resemblance to intuition from linear theory. First, we note that despite their obvious differences, boxes Example, AGB, H II-near, and $\mathrm{H}$ II-far have several qualitative properties in common. These runs all have $\beta \gtrsim 1$, and $\tau \lesssim 100$, a value that is not too large. They all share a defining feature, that the dust is strongly 'clumped' and remains highly anisotropic even in saturation. Prominent clumps, plumes, filaments, and sheets appear, even when the gas is only weakly compressible.

In these, the 'medium' and 'large' boxes (Example, AGB M/L/XL, H II-near M/L, H II-far M/L) saturate with significant anisotropy or bias in $\delta \mathbf{u}_{\mathrm{g}}$ along the direction of the acceleration a (as opposed to e.g. $\mathbf{w}_{\mathrm{s}}$ or $\mathbf{B}$ ). The components in the perpendicular direction are not negligible and the strength of the anisotropy varies, owing to mixing from the Lorentz forces. These runs also generally have

$|\delta \mathbf{B}| /|\mathbf{B}| \sim\left|\delta \mathbf{u}_{g}\right| / v_{A}$

(kinetic/magnetic energies similar) with anisotropies oriented in the same plane(s). Moreover

$\left|\delta \mathbf{v}_{d}\right| \sim\left|\delta \mathbf{u}_{g}\right|$,

with $\delta \mathbf{v}_{\mathrm{d}}$ typically slightly larger, but not by more than a factor of a few. In the largest boxes, $\delta \rho_{\mathrm{d}} / \rho_{\mathrm{d}} \sim \delta \rho_{\mathrm{g}} / \rho_{\mathrm{g}}$, (Fig. 10), and the PDFs become increasingly Gaussian/lognormal (Figs 22 and 23) especially at high- $\mu$ (Fig. 18). In the intermediate-size-scale (M) boxes $\delta \rho_{\mathrm{d}} / \rho_{\mathrm{d}}>\delta \rho_{\mathrm{g}} / \rho_{\mathrm{g}}$.

These behaviours can all, remarkably, be predicted (at least qualitatively) by the linear properties of the fastest-growing modes at the box scale. These predictions are discussed in detail in Hopkins $\&$ Squire (2018b, fig. 2) and Paper I (sections 4-5), and we briefly summarize them here. If the relevant modes at the box scale are

\footnotetext{
${ }^{20}$ This follows from the linear RDI behaviour and can be understood from a local-balance-type argument from the equations for 'forcing' the dust via gas. Dimensionally, a linear perturbation $\delta \mathbf{v}_{d}$ should have $\omega \delta \mathbf{v}_{d} \sim \delta \mathbf{u}_{g} / t_{0}$ where $t_{0} \approx t_{s}$ if drag dominates, or $t_{0} \approx t_{L}$ if Lorentz forces dominate, so $\left|\delta \mathbf{v}_{d}\right| \sim\left|\delta \mathbf{u}_{g}\right| /\left(\omega t_{0}\right)$. But generically for the MHD-wave RDI-type modes, $\omega \sim t_{0}^{-1}\left(\mu k c_{s} t_{0}\right)^{v}$ with $v \sim 1 / 3-2 / 3$ depending on wavelength, so the scaling switches from $\left|\delta \mathbf{v}_{d}\right| \gtrsim\left|\delta \mathbf{u}_{g}\right|$ to $\left|\delta \mathbf{v}_{d}\right| \lesssim\left|\delta \mathbf{u}_{g}\right|$ around $k \sim 1 /\left(\mu c_{s} t_{0}\right)$.
}

the MHD-wave RDI modes or the aligned modes $\left(\hat{\mathbf{k}}=\hat{\mathbf{w}}_{s}\right.$, also called 'pressure-free' or quasi-sound/drift modes), then for modest magnetization ( $\beta^{-1}$ and $\tau$ not too large) most of the insight can be gained from considering the much simpler pure hydro case (see Paper I for further discussion). At $k \lesssim \mu / c_{s} t_{s}$ (AGB L/XL and H II-near L, where $\left.L_{\text {box }} / c_{s} t_{s} \gtrsim 1000\right)$, the aligned 'pressure-free' mode dominates, where internal pressure effects of the gas are weak compared to the bulk force from dust on gas. In this mode $\delta \rho_{\mathrm{d}} /\left\langle\rho_{\mathrm{d}}\right\rangle \approx \delta \rho_{\mathrm{g}} /\left\langle\rho_{\mathrm{g}}\right\rangle$ and $\delta \mathbf{v}_{\mathrm{d}} \approx \delta \mathbf{u}_{\mathrm{g}}$ (i.e. dust and gas fluctuate together; see Fig. 10), with $\delta \mathbf{u}_{g} \propto \hat{\mathbf{k}}$ (fluctuations are longitudinal) and maximum growth rates at $\hat{\mathbf{k}}=\hat{\mathbf{w}}_{s}$. Because of the weak pressure effects, $\mathbf{B}$ is driven passively by the velocity fluctuations so $|\delta \mathbf{B}| /|\mathbf{B}|$ $\sim\left|\delta \mathbf{u}_{\mathrm{g}}\right| / v_{\mathrm{A}}$ (with $\delta \mathbf{B}$ orthogonal to $\delta \mathbf{u}_{\mathrm{g}}$ in the $\mathbf{B}-\delta \mathbf{u}_{\mathrm{g}}$ plane). ${ }^{21}$ Note that initially, $\hat{\mathbf{w}}_{s}$ is not aligned with $\mathbf{a}$ (for non-zero $\tau$ ), so the modes produce the 'sheets' of overdensity in dust perpendicular to $\hat{\mathbf{w}}_{s}$. However, because the pressure effects are weak in these modes, the non-linear forcing from a tends to overwhelm competing forces like magnetic tension, and push the system to drift in the â direction (giving $\left.\delta \mathbf{u}_{\mathrm{g}} \propto \mathbf{a}\right)$.

In the 'intermediate' boxes (Example, AGB, H II-near, and $\mathrm{H}_{\text {II- }}$ far M) the 'mid- $k$ ' MHD-wave modes dominate at $\mu \lesssim k c_{s} t_{s} \lesssim$ $\mu^{-1}$. Again the linear modes have $\delta \mathbf{v}_{\mathrm{d}} \sim \delta \mathbf{u}_{\mathrm{g}},|\delta \mathbf{B}| /|\mathbf{B}| \sim\left|\delta \mathbf{u}_{\mathrm{g}}\right| / v_{\mathrm{A}}$. At mid- $k$, the initially fastest-growing modes approximately satisfy $\hat{\mathbf{k}} \perp \mathbf{B}$ if $\left|\mathbf{w}_{\mathrm{s}}\right| \ll c_{\mathrm{s}}$ (the Alfvén or slow RDIs) or $\hat{\mathbf{k}} \perp \hat{\mathbf{w}}_{s}$ if $\left|\mathbf{w}_{\mathrm{s}}\right| \gg c_{\mathrm{s}}$ (the fast RDI). This produces the perpendicular sheets and filaments extended along $\hat{\mathbf{w}}_{s}$, which are seen at early times. It also explains the observed anisotropies, although these are weaker because the linear modes have a mix of components in each direction. Perhaps most notably, these boxes have $\delta \rho_{\mathrm{d}} /\left\langle\rho_{\mathrm{d}}\right\rangle \gg \delta \rho_{\mathrm{g}} /\left\langle\rho_{\mathrm{g}}\right\rangle$, which, as shown in Paper I, are likely related to the linear modes, which satisfy $\delta \rho_{d} \sim \delta \rho_{g} \Im\left(\omega t_{s}\right)$, i.e. $\delta \rho_{d} /\left\langle\rho_{d}\right\rangle \approx\left(\Im\left(\omega t_{s}\right) / \mu\right) \delta \rho_{g} /\left\langle\rho_{g}\right\rangle$. Because $\Im\left(\omega t_{s}\right) \sim\left(k c_{s} t_{s}\right)^{1 / 2}$ in this mode, the relative strength of $\delta \rho_{\mathrm{d}} / \delta \rho_{\mathrm{g}}$ increases at smaller scales and smaller $\mu$, consistent with our experiments (Figs 10, 28, 29). While this provides a reasonable qualitative explanation for the observed trends, we do caution that the magnitude of the saturated $\delta \rho_{\mathrm{d}} / \delta \rho_{\mathrm{g}}$ is often significantly larger than that predicted by linear theory.

In the smaller boxes (AGB S, H II-near S, H II-far S), the 'high$k$ 'MHD-wave modes dominate, with $k \gtrsim 1 /\left(\mu c_{s} t_{s}\right)$. The fastestgrowing mode directions are the same as the 'mid- $k$ ' modes (Paper I), with a similar anisotropy (here $\delta \mathbf{v}_{d} \propto \delta \mathbf{u}_{g} \propto \hat{\mathbf{k}}$ to leading order, giving anisotropy in the $x y$ plane for $\delta \mathbf{u}_{\mathrm{g}}$, and in $z$ for $\delta \mathbf{B}$ ). Again $|\delta \mathbf{B}| /|\mathbf{B}| \sim\left|\delta \mathbf{u}_{\mathrm{g}}\right| / v_{\mathrm{A}}$ in the linear mode and saturated turbulence (Table 2). As in the mid- $k$ modes, we have $\delta \rho_{d} \sim \delta \rho_{g} \Im\left(\omega t_{s}\right)$, but now with $\Im\left(\omega t_{s}\right) \sim\left(k c_{s} t_{s}\right)^{1 / 3} \gtrsim 1$, so the ratio $\delta \rho_{\mathrm{d}} / \delta \rho_{\mathrm{g}}$ continues to rise with smaller $\mu$ or at smaller $k$ (making $\delta \rho_{\mathrm{d}}$ weakly dependent on box size, with a small decrease to smaller $L_{\text {box }}$ ). This occurs even though $\delta \rho_{\mathrm{g}} \rightarrow 0$ (see Fig. 10). One notable difference from the mid$k$ modes, however, is that the linear perturbations feature $\left|\delta \mathbf{v}_{d}\right| \sim$ $\left|\delta \mathbf{u}_{g}\right| / \Im\left(\omega t_{s}\right) \ll\left|\delta \mathbf{u}_{g}\right|$. This feature is also seen in the saturated turbulence (Fig. 10).

We stress that despite their common elements, there are important differences across these clumped boxes, beyond just the magnitude

\footnotetext{
${ }^{21}$ For example, if we assume $\delta \mathbf{u}_{g} \propto \mathbf{a} \propto\left(\sin \theta_{\mathbf{B a}}, 0, \cos \theta_{\mathbf{B a}}\right)$ in $(\hat{x}, \hat{y}, \hat{z})$, then since $\hat{\mathbf{B}}=\hat{z}$, for linear perturbations $\delta \mathbf{B} \propto\left(-\cos \theta_{\mathbf{B a}}, 0, \sin \theta_{\mathbf{B a}}\right)$. So for our runs with $\left|\mathbf{B}_{0} \cdot \mathbf{a}\right|=\cos \theta_{\mathbf{B a}}=1 / \sqrt{2}$, this gives $\delta \mathbf{u}_{g} \propto$ $(1 / \sqrt{2}, 0,1 / \sqrt{2})$ and $\delta \mathbf{B} \propto(-1 / \sqrt{2}, 0,1 / \sqrt{2})$ (i.e. the absolute magnitude of the anisotropy is the same in each direction for $\delta \mathbf{u}_{g}$ and $\delta \mathbf{B}$ ). For our runs with $\left|\mathbf{B}_{0} \cdot \mathbf{a}\right|=0.05$, this gives $\delta \mathbf{u}_{g} \propto(0.99,0,0.05)$ and $\delta \mathbf{B} \propto(-0.05,0,0.99)$ (so the dominant direction for $\delta \mathbf{u}_{g}$ is $\hat{x}$, while that for $\delta \mathbf{B}$ is $\hat{z}$ ). These compare well to the results in Table 2.
} 
of the effects. The morphology, topology, and even dimensionality (e.g. clumps, filaments, sheets) of the dust structures varies and depends on a complicated mix of both the global parameters (e.g. $\left|\mathbf{w}_{\mathrm{s}}\right| / c_{\mathrm{s}}, \tau, \beta$, etc.), as well as scale, owing to the complex superposition of different modes. Box AGB, with initial $\tau \ll 1$, is closest to the pure-hydrodynamic cases studied in Moseley et al. (2019). As a result it saturates in primarily compressible, supersonic magnetosonic turbulence, with the saturation amplitudes for $\mathbf{u}_{\mathrm{g}}$ in boxes M/L/XL well-predicted by the eddy turnover time argument (tested in detail therein), and $\delta \mathbf{B}$ following from $\delta \mathbf{u}_{\mathrm{g}}$. Box Example, with higher $\tau \sim 30$, saturates in primarily incompressible MHD turbulence (see Seligman et al. 2019). In this case, the saturation amplitude of $\delta \mathbf{B}$ (especially its variations with $\mu$ ) is more accurately predicted by assuming force balance between forcing from dust and magnetic tension of the dominant (box-scale) modes, with $\delta \mathbf{u}_{\mathrm{g}}$ following from $\delta \mathbf{B}$, i.e.:

$\frac{(\mathbf{B} \cdot \nabla) \mathbf{B}}{4 \pi} \sim \frac{|\mathbf{B}||\delta \mathbf{B}|}{4 \pi L_{\mathrm{box}}} \sim \frac{\rho_{d} \mathbf{w}_{s}}{t_{s}} \approx \rho_{d} \mathbf{a}$

(see Fig. 10 and Seligman et al. 2019).

Although it is beyond the scope of this work to study cases where the external drift driving these instabilities initially is time variable, it is worth noting that even if that drift were somehow 'turned off' (which should allow the induced turbulence to decay), there is no obvious mechanism to 'disperse' the dust-to-gas fluctuations formed. Also, in environments with some externally driven turbulence, it would be interesting to explore whether the net effect of this turbulence is to enhance the dust-to-gas ratio fluctuations (as occurs in the absence of RDIs; see Hopkins \& Lee 2016; Lee et al. 2017) or to limit the saturation of the RDI-induced clumping.

\subsection{Saturation: 'dispersed' or 'granular' states}

We refer to the second regime as 'Dispersed' or 'Granular', because the dust is generally more dispersive in these runs (boxes CGM, WIM, Corona). It appears that the transition between the two regimes occurs as $\tau$ becomes very large, specifically $\tau \gtrsim 100$. In this regime, the saturated states of the instabilities begin to differ from the description above, and the dust has more isotropic velocity dispersion and notably smaller density fluctuations (especially at the high- $\rho_{\mathrm{d}}$ end, which is suppressed relative to low- $\rho_{\mathrm{d}}$; see Figs 25 27).

Many of the saturated properties are consistent with the dominant linear modes, as observed in the previous regime. Unlike the 'clustered' boxes, which are dominated by a combination of the low- $k$ 'pressure free' (and quasi-sound/drift) modes and mid/high- $k$ MHD-wave modes, at sufficiently high- $\tau$ the instabilities become increasingly confined along B. Boxes CGM, WIM, and Corona are dominated by a combination of the strong $\mathbf{B}$-aligned 'cosmic raylike' instabilities (see Paper I for details), together with the related gyro RDIs in WIM S and Corona S/M (Fig. 2). In linear theory, the fastest-growing modes (in both cases) have

$\hat{\mathbf{k}} \approx \hat{\mathbf{B}}$

with $\mathbf{B}$ field fluctuations transverse ( $\delta \mathbf{B}$ preferentially in the $x y$ plane, similar to an Alfvén wave), with ${ }^{22}$

$|\delta \mathbf{B}| /|\mathbf{B}| \sim\left|\delta \mathbf{u}_{g}\right| / v_{A}$.

\footnotetext{
${ }^{22}$ As discussed in Fig. 7, WIM L (the default run, with larger drift velocity) is the one notable exception with $|\delta \mathbf{B}| /|\mathbf{B}| \ll\left|\delta \mathbf{u}_{\mathrm{g}}\right| / v_{\mathrm{A}}$.
}

Like the 'clumped' case the gas fluctuations $\delta \rho_{\mathrm{g}}$ can be related to $\delta \mathbf{u}_{\mathrm{g}}$ with the usual MHD turbulence scalings. However, the anisotropy is often weak, because (1) the overall turbulence is isotropized and (2) the linear modes have components in all directions. Also like the 'clumped' case, the intermediate/large-scale boxes (CGM $\mathrm{M} / \mathrm{L}$, Corona M/L, WIM L) have a saturation amplitude of the gas that is reasonably well explained by equating eddy turnover and growth time-scales $\left(\delta \mathbf{u}_{g} \sim \Im\left(\omega\left[L_{\text {box }}\right] L_{\text {box }}\right)\right),{ }^{23}$ while the smallscale $^{24}$ boxes (Corona S, WIM S/M) have a saturation amplitude $\delta \mathbf{u}_{\mathrm{g}}$ that is better explained by the same 'high- $k$ ' scaling as the 'clumped' cases above (see final paragraph of Section 5.3).

In the linear modes of these high- $\tau$ cases, the $\mathbf{v}_{\mathrm{d}}$ perturbation is approximately a gyro orbit, i.e. preferentially equal power in the $x y$ direction. The scaling of $\delta \mathbf{v}_{\mathrm{d}} / \delta \mathbf{u}_{\mathrm{g}}$ is similar to the 'mid- $k$ ' and 'high$k$ ' MHD-wave cases discussed above (i.e. substantially smaller in the high- $k$ limit) but enhanced by a factor between $\sim \mu^{-1 / 2}$ and $\mu^{-1}$ (depending on wavelength in the out-of-resonance gyro or cosmic ray like mode; see Paper I, section 4). This is directly evident in Fig. 10, which shows that $\left|\delta \mathbf{v}_{\mathrm{d}}\right| /\left|\delta \mathbf{u}_{\mathrm{g}}\right| \gtrsim 1$ in many of these strongly magnetized cases. Finally, $\delta \rho_{\mathrm{d}}$ also scales qualitatively like the mid/high- $k$ MHD-wave modes, in that it is weakly dependent on $L_{\text {box }}$ or $k$ (while $\delta \rho_{\mathrm{g}}$ decreases at lower $L_{\mathrm{box}}$ ). However, in both the aligned cosmic ray-like and gyro modes, the Lorentz motion is (to leading order) incompressible, with the dust density fluctuations suppressed by a factor $\sim \mu^{1 / 2}$ and gas density fluctuations by a factor $\sim \mu$ (see Paper I, section 6.4). This suggests that $\delta \rho_{\mathrm{d}} \sim \exp \left(\delta \ln \rho_{\mathrm{d}}\right)$ -1 should be an order-of-magnitude lower compared to similar 'clumped' runs. This intuition provides a surprisingly good fit to the difference between 'clumped' and 'dispersed' runs in Fig. 10.

This regime is analogously very broad, and there is no single, transcendent behaviour that defines it. In Corona M/L and CGM, the aligned modes initially produce rather thick 'sheets' in the $x y$ plane perpendicular to $\mathbf{B}$. These form as dust particles move slowly relative to each other along the field lines, and collapse into thin sheets, with an increase in $\delta \rho_{\mathrm{d}}$. However, once the sheet becomes thin, the acceleration on dust in direction $\hat{\mathbf{a}} \neq \hat{\mathbf{B}}$ pushes the dust with a component transverse to $\mathbf{B}$ only at one point along $\mathbf{B}$. This excites gyro motion of dust about $\mathbf{B}$, but also drags the field and 'bends' $\mathbf{B}$ locally (as opposed to simply pushing the entire field line uniformly, as in the initial state), generating a magnetic tension and exciting Alfvén waves. That, in turn, can re-orient the gyro motion (bending or 'dispersing' the sheet). How 'isotropized' the dust is and, correspondingly, how uniformly the dust is spread - depends on how easily the field can be bent. Thus in CGM, with high- $\beta$, the fields and corresponding $\mathbf{v}_{\mathrm{d}}$ can be fully isotropized; in contrast, in Corona-M, the low- $\beta$ and small scales mean the energy in the dust cannot fully re-orient the fields, and the dust motion remains primarily in the $x y$ plane. The maximum dust velocity dispersion is

\footnotetext{
${ }^{23}$ The notable apparent exceptions in Fig. 10 are the CGM low- $\tau_{\text {low }}$ runs. However, Fig. 13 shows this initially grows vigorously at high $k\left(\sim 100 / L_{\text {box }}\right)$ and in fact it reaches a large $\left|\delta \mathbf{u}_{\mathrm{g}}\right| \sim c_{\mathrm{S}}$, before the 'sheets' breakup and disperse the dust suppressing growth of larger scale modes, and $\delta \mathbf{u}_{\mathrm{g}}$ actually decays somewhat before reaching its equilibrium value. If we use the higher $k$ at which the largest rapidly growing modes are present, and the larger $\mathbf{u}_{\mathrm{g}}$, before the isotropized dust orbits lead to less coherent turbulent motions in the gas, then these runs are plausibly consistent with the "eddy turnover time' saturation scaling.

${ }^{24}$ As discussed in Paper I, when $\tau \gg 1$, the dividing line between 'small' and 'intermediate' scales is not simply $k \gtrsim 1 /\left(\mu c_{s} t_{s}\right)$, but can become a rather complicated expression of $\tau, \beta$, etc. Here, they can be effectively defined by the presence in Fig. 2 of modes with the 'high- $k$ ' asymptotic scalings.
} 
set by equating the 'pumping' of the gyro motion (acceleration a) with damping by drag, which just gives

$\left|\mathbf{v}_{d}\right| \sim|\mathbf{a}| t_{s} \sim\left|\mathbf{w}_{s}\right|$,

with isotropic $\mathbf{w}_{\mathrm{s}}$.

In contrast, in Corona S, and WIM S/M, the dust is collected in dense 'lines', or, more precisely, closed vertical sheets or 'tubes', oriented along the $\mathbf{B}$ direction. The dominant modes are gyro modes (not the aligned modes), so the 'horizontal sheets' discussed above do not form. ${ }^{25}$ This produces the 'granular' appearance of $x y$ slices. Without the 'sheets' to bend the fields strongly at individual points, the field lines move coherently in aligned 'columns' or 'tubes'. Therefore, the turbulence is essentially two-dimensional, and because the scales are small the gas is weakly compressible. These features are also evident in box WIM-L, although this also has some aligned modes, giving it a mix of properties.

It is also worth noting that in some boxes (e.g. Corona-S), the modes do not appear to reach the grid scale, even after the simulation has been evolved for longer than the box-scale mode growth time. This tends to occur when there are strongly growing, dominant gyro modes on somewhat smaller scales which can retain their dominance, even at late times. There is some hint that these may continue growing, along with box-scale modes, more slowly (e.g. linearly in time). Unfortunately it is computationally prohibitive to run these boxes to arbitrarily long times.

We emphasize that there does not appear to be a sharp 'threshold' where behaviour changes between 'clumped' and 'dispersed' modes. Obviously, some 'clumped' runs feature gyro modes and closer-to-isotropic grain velocity dispersions, while some 'disperse' runs here (like Corona-M) retain coherent dense grain structures well into their non-linear evolution. Rather, there is a spectrum of different behaviour in different regimes, and different parameters involve a different mixture of these.

\section{OTHER PHYSICS (NOT INCLUDED HERE)}

The simulations here are intentionally idealized, designed to study the physics and non-linear behaviours of the instabilities identified in Paper I. Of course, in different specific physical applications, there are an infinite variety of additional physics which could also be important, some of which may modify the instabilities themselves. Some of these cases will be studied in future work modelling observables in specific physical systems (e.g. AGB outflows and dust extinction/density estimation; Steinwandel et al., in preparation), or analytic studies focused on the linear instabilities in more complex systems (e.g. three-fluid radiation-MHD RDIs, Squire et al., in preparation). Other examples (viscosity, non-ideal MHD, stratification, differential rotation) are discussed in detail in Hopkins \& Squire (2018a,b) and Squire \& Hopkins (2018a). But we briefly review some here for context.

Small-scale transport processes (e.g. viscosity, conductivity) are discussed in Paper I. For the physical examples which motivate our parameter study in Table 1 , we specifically chose the parameters

\footnotetext{
${ }^{25}$ We have checked that there is no apparent correlation between the presence of these tubes and whether or not the box is large enough that the dust 'wrapping' time $\left(L_{\mathrm{box}} / \mathbf{w}_{\mathrm{s}}\right)$ is shorter or longer than the mode growth time, which might artificially contribute to such structures. We have also checked for any dependence on whether the dust gyro radii are resolved or unresolved in the gas cells (because of our superparticle approach, dust orbits are always resolved).
}

so that our smallest resolvable scales in the smallest (' $\mathrm{S}$ ') boxes correspond to the scale where viscous effects could begin to become significant ( $k^{2} \gtrsim|\omega| / \nu$, where $v$ is the kinematic viscosity), so that all resolved scales in all our boxes correspond to the 'inertial range' where viscous effects are weak (see section 9 in Paper I, where these scales are estimated). But we also note, as shown explicitly in Paper I (e.g. fig. 7 therein and Squire \& Hopkins 2018a), that most of the instabilities here are undamped below the viscous scale (down to at least the ion gyro-radii), like e.g. the cosmic ray resonant and nonresonant instabilities (Wentzel 1968; Bell 2004). We also choose motivating parameters where non-ideal MHD effects should be negligible (these terms generally require an ionization fraction $f_{\text {ion }}$ $\ll 10^{-8}$ to be significant for the modes studied here; see section 8.3 in Paper I), but note that even in overwhelmingly neutral gas (e.g. protoplanetary discs), ambipolar diffusion and the Hall effect do not actually damp many of the instabilities studied here (but instead modify them into additional branches; see e.g. Squire \& Hopkins 2018a, figs 7-8). Other small-scale effects such as the current carried by dust grains (section 8.3 in Paper I), finite inter-grain separation effects, or degeneracy pressure are completely negligible in any regime motivating our study here, or any regime where ideal MHD could actually apply (e.g. the dust current is subdominant by a factor $\sim 10^{10} f_{\text {ion }}\left(T / 10^{4} \mathrm{~K}\right)$ in the induction equation).

On the largest spatial scales, global effects such as stratification, gradients in the external forces driving drift, rotation, and shear make our local periodic-box approximation invalid. We have therefore specifically chosen parameters such that the largest scales in our largest boxes ('L/XL') in Table 1 correspond to these scales. All of these global effects introduce new RDIs such as the BruntVäisälä, vertical settling, and epicyclic RDIs (see e.g. appendix C in Hopkins \& Squire 2018b or sections 5 and 6 in Squire \& Hopkins 2018a).

There are also various effects which directly involve dustgas interactions and operate on relatively long time-scales such as gas cooling; dust accretion, mantle formation, and chemical enrichment (generally relevant in cold or very dense gas like AGB outflows); or sputtering of dust in hot gas (relevant in gas with $\left.T \gtrsim 10^{6} \mathrm{~K}(\mu / 0.01)\right)$. These can modify the equation of state of the gas, dust-to-gas ratios or dust size/charge, but generally operate on time-scales much longer than (or at most comparable to) the RDI growth times. It is therefore unlikely that these strongly modify the RDIs directly: instead a medium with e.g. efficient cooling or sputtering would simply shift the parameters of interest here, having effectively lower temperature or dust-to-gas ratios, respectively. But it is likely that these processes are themselves strongly modified by the RDIs, as they depend sensitively on clumping factors of the dust and gas and their (grain-size-dependent) cross-correlation, a subject worthy of future study.

One effect which could modify the instabilities here is externally driven turbulence. Although we clearly show the RDIs persist in the presence of self-excited turbulence, it is possible that if some other process drives gas turbulence much more strongly (on a given scale) it could significantly modify the RDIs. This could potentially set the saturation amplitude by e.g. shearing apart different modes if the eddy turnover times become much shorter than RDI growth times (see Squire \& Hopkins 2018a, section 8), although it is also well-known from both theory and laboratory experiments that even test-particle grains ( $\mu=0$, so no RDIs are present) are actually strongly clustered on small-scales in externally driven turbulence (see e.g. Squires \& Eaton 1991; Fessler et al. 1994; Bracco et al. 1999; Cuzzi et al. 2001; Rouson \& Eaton 2001; Monchaux et al. 2010; Pan et al. 2011; Hopkins \& Lee 2016). This is most likely 
to be important in physical systems motivating boxes like WIM or CGM: $\tau_{\text {low }}$, where strength of the saturated gas turbulence from the RDIs alone (Table 2) is significantly weaker than the level of turbulence observed.

A variety of physical effects will also become important specifically when the local dust-to-gas ratio $\mu$ becomes very large. The most obvious is grain-grain collisions, as the ratio of stopping to collision time-scales as $t_{\text {stop }} / t_{\text {collision }} \sim \mu\left(\delta v_{\mathrm{dd}} / c_{s}\right)$ (where $\delta v_{\mathrm{dd}}$ is the rms relative grain-grain velocity difference on infinitesimally small spatial scales). Obviously this will become important at the very high $\mu$ reached by some of our simulations on small scales, but how it modifies the distribution of $\mu$ itself and subsequent RDIs depend on the outcome of said collisions (which can produce grain growth/coagulation, elastic or inelastic scattering/bouncing, or grain shattering, depending sensitively on the local conditions and grain chemistry). In some cases, the self-gravity of a cluster of grains can become important before collisions (though this is more likely important in protoplanetary discs, it could occur under exceptional ISM conditions, see e.g. Hopkins 2014). And if radiation pressure drives the dust drift, then the dust can become self-shielding if the dust-to-gas ratio within a patch of size $\sim \lambda$ exceeds $\mu(\lambda) \gg \tilde{\alpha}\left(\lambda / L_{\text {box }}\right)^{-1}\left(1+\lambda_{\text {rad }} / \epsilon_{\text {grain }}\right)$ (where $Q \sim(1+$ $\left.\lambda_{\text {rad }} / \epsilon_{\text {grain }}\right)^{-1}$ crudely approximates the grain absorption efficiency for incident radiation with wavelength $\lambda_{\text {rad }}$ ). In that regime the radiation can be multiply scattered, leading to more complicated three-fluid (radiation, dust, gas) RDIs. The very high local $\mu$ reached here in the absence of these effects should be considered motivation for studying these different regimes.

\section{SUMMARY AND CONCLUSIONS}

We have presented the first simulation parameter study of the nonlinear regime of the Squire \& Hopkins (2018b) 'RDIs' of charged dust grains in magnetized gas. Because the parameter space of the instabilities is large and this is a first study, we focused on several sets of initial conditions that are broadly representative of different astrophysical regimes. In all cases studied, the instabilities produce highly non-linear structures and fluctuations, often including strong turbulence and magnetic field amplification. Strong anisotropy and non-linear features appear in the dust, including orbit crossing, density fluctuations, and complicated velocity distribution functions. This necessitates numerical simulations using dust particle methods which can follow the full non-linear dust velocity distribution function, as opposed to just the fluid limit.

Even within our small survey, the simulations exhibit a diverse array of behaviours. In Paper I, we demonstrated using linear perturbation theory that a homogeneous MHD gas coupled to dust via Lorentz forces and drag exhibits around 10 different 'instability families'. These families include the Alfvén, slow, and fast magnetosonic MHD-wave RDIs; the three corresponding gyro RDIs; the 'pressure-free' and related 'quasi-drift' and 'quasi-sound' modes; and the 'cosmic ray streaming'-type modes. These families all have different linear growth rates and mode structures, but often overlap and occur within the same system. Without introducing additional physics or constraints (e.g. Braginskii viscosity, which suppresses the growth rate of the magnetosonic modes, but not the Alfvén modes), it is generally impossible to construct a simulation that isolates a single instability family. Our range of initial conditions were chosen both to be representative of different astrophysical regimes and also to exhibit different dominant, fastest growing instability families. The resultant non-linear evolution yields a remarkable diversity of outcomes.
We show that over the course of the simulations, the instabilities become violently non-linear on all scales. Their non-linear outcomes can result in dust being highly concentrated (the 'clumped' regime), or dispersed with large isotropic velocity dispersions (the 'disperse' regime). In the 'clumped' case, there are a wide range of morphologies, topologies, and dimensionalities of the clumped structures, depending on the parameters of the system, with dust in e.g. multiply connected sheets, filaments, or point-like clumps. In the most extreme cases, the dust can reach enormous overdensities in these idealized tests $\left(>0.1\right.$ per cent of the dust mass at $\gg 10^{9}$ times the mean dust density, with volume-averaged 'clumping factors' $>10^{4}$ ). The dust clumping does not depend systematically on the spatial scale or the compressibility of the gas: clumping can be stronger on small scales in nearly incompressible gas than on large scales in highly compressible cases. Surprisingly, the clumping is stronger at lower dust-to-gas ratios, although the growth times of the instabilities are longer.

In contrast, in the 'disperse' cases, the dust can be accelerated to highly supersonic isotropic velocity dispersions (even undergoing first-order Fermi acceleration with stretched-exponential velocity 'tails' that would reach relativistic velocities in some physical systems) and dispersed nearly uniformly over the box (clumping factors as small as $\sim 1.02$ ). These cases are akin to well-studied cosmic ray instabilities that self-excite diffusive behaviour (i.e. selfgenerating dust diffusion). The growth times of these instabilities can be shorter than either the dust drag/stopping or gyro times, and are often extremely short relative to other time-scales in the gas (e.g. the sound-crossing or dynamical times, for physical systems of interest). This will have a huge range of important physical ramifications for essentially all regimes where dust is present.

The dust drives anisotropic turbulence in the gas, whose properties may be qualitatively understood via heuristic quasi-linear theory. The gas turbulence is stronger and more compressible if the box scale is larger, or if the dust-to-gas ratio is larger. Moreover, the gas velocity and density fluctuations are correlated in approximately the same manner observed for pure-gas MHD turbulence. In the saturated state, the instabilities tend to produce equipartition between gas velocity and magnetic field fluctuations. The latter means that systems can have the magnetic fields strongly amplified by the instabilities (e.g. decreasing $\beta$ by factors of $\sim 10^{4}$, in the most extreme cases here).

The gas turbulence can be qualitatively magnetosonic or Alfvénic, but in addition to the dust properties, it has many characteristics that are unique. PDFs of various fluctuations $\left(\mathbf{u}_{\mathrm{g}}\right.$, $\left.\mathbf{B}, \rho_{\mathrm{g}}, \mathbf{v}_{\mathrm{d}}, \rho_{\mathrm{d}}\right)$ are typically highly non-Gaussian, with exponential or stretched-exponential tails indicative of strong intermittency and stochastic driving in a highly dissipative system. These large tails mean that sometimes the mass and volume-weighted statistics can yield quite different results, and the tails can be many orders-ofmagnitude more populated than Gaussian (e.g. many of our systems have $>0.1$ per cent of their dust or gas mass at $\gtrsim 10-20$ 'standard deviations' in some property).

The parameter space here is highly multidimensional, so it is difficult to make conclusions that can be robustly applied to all regimes. However, it appears that choices such as the detailed equation of state, form of the dust charge scaling with ambient gas properties, exact magnetic $\beta$, initial direction of field orientation (relative to the relative dust-gas acceleration), or exact dust-to-gas ratio do not dramatically (qualitatively) change the character of the solutions. As anticipated from linear theory in Paper I, the most important parameters that determine the qualitative behaviour appear to be the physical scale and ratio of 
magnetic (Lorentz) to drag (aerodynamic + Coulomb) forces on dust.

Future work will be necessary to investigate how these instabilities will manifest in physical systems. We stress that the models here are intentionally idealized: we follow a single grain species subject to a constant external differential dust/gas acceleration in periodic, initially homogeneous gas boxes, with an ideal equation of state. Therefore, our model names (e.g. AGB) should not be taken literally - these are not intended to be realistic physical simulations of those systems. Rather, the names are chosen reflect an example of a system where the key dimensionless parameters for these instabilities $(\tau$, $\left.\beta,\left|\mathbf{w}_{\mathrm{s}}\right| / c_{\mathrm{s}}\right)$ are similar to the box simulated. Nor is it obvious, yet, how the simulations here directly translate to observational consequences: even if the behaviour in fully 'realistic' systems is similar to that predicted here, this will clearly have consequences for dust extinction curves and emission, cooling and dust chemistry, and many other areas, but the magnitude or even the sign of these effects depends sensitively on the exact observations considered, as well as physical conditions (e.g. chemical conditions, optical depth, additional radiative transfer effects) beyond those modelled here. The physical systems simply provide helpful motivation for our survey and in future work we will explore more realistic scenarios.

This investigation has begun to elucidate the broad non-linear behaviours of the resonant drag instabilities in magnetized gas. However, it raises more questions than it answers, some of which include:

(i) What are the effects of a broad spectrum of grain sizes and charges? Here, we intentionally simulate a single species of grains, so that the growth rates and dominant modes can be clearly defined and studied. However, in almost all astrophysical situations there will be a wide range of grain sizes. In some circumstances the largest grains (which tend to dominate the dust mass) will dominate the dynamics, while in others there is an intricate mix of which grains dominate which terms in the relevant equations (for a more detailed discussion, see Paper I). Further simulations will be required to understand when different grain sizes are effectively independent, and when they will have strong non-linear interactions via the gas (see e.g. Bai \& Stone 2010a, for examples in the streaming RDI).

(ii) Is there a meaningful way to define convergence, and incorporate the effects of all relevant size scales in a single simulation? The dynamic range over which the instabilities are present with interesting growth rates is enormous, and far larger than can be simulated at present. Because we find that the gas turbulence has most of its power at the largest (driving) scales, there is hope that, like MHD turbulence, certain bulk properties (e.g. the bulk power in turbulence, the dissipation rate, and most of its effects) can be 'converged' even if the Kolmogorov scale is unresolved. It is unclear if a meaningful convergence criteria can even be defined for the dust, since some properties, like the dust density fluctuations, are not uniquely dominated by the driving-scale modes.

(iii) What is the effect of stratification or time variability on the instabilities? As the size scale of the simulations increases, the physical system would become stratified and non-uniform in space or time. We have shown that stratification and other large-scale terms such as shear, rotation, and differential acceleration (e.g. Coriolis forces) can all introduce additional instabilities, some of which have faster growth rates on large scales than those here (see Squire \& Hopkins 2018a). So, this could introduce unique and important phenomena. On large time-scales, the 'forcing' terms driving dust drift (hence the instabilities) could fluctuate: if this occurs rapidly compared to growth time-scale it may produce unique phenomena as well. It would be particularly interesting to study the analogue of 'decaying turbulence' when external acceleration/forcing is suddenly 'turned off' once the simulations have reached saturation.

(iv) What is the nature of the non-linear gas turbulence and its intermittency? Any of these simulations provide ample opportunity to study the nature of the turbulence. We have only explored simple, 'zeroth-order' diagnostics, but more detailed studies of the Eulerian and Lagrangian power spectra and structure functions will provide substantial insight into the structure of the turbulence. The highly non-Gaussian behaviour we see suggests that the turbulence may be quite different from a simple Kolmogorov-type picture (although of course pure-MHD turbulence is already substantially non-Gaussian in some measures).

(v) Can we develop a predictive theory for the dust clumping and turbulence? Here, we are able to develop some qualitative insights into when dust should exhibit clustering. Further, from quasi-linear theory, we can heuristically understand why the dust density fluctuations become stronger at lower dust-to-gas ratio, and are weakly dependent on the spatial scale or compressibility of the gas. However, this is far from a predictive or quantitative theory. Most past work on the non-linear saturation of particle clustering in turbulence assumed 'passive' grains (i.e. neglected the forces of dust on gas). However, in the 'passive' case these instabilities do not exist, and the predicted saturated dust clustering (neglecting backreaction terms) can be very different from what we find here. It is clearly important to develop new theoretical models which can explain the strength and nature of the observed dust structures.

(vi) What are the important regimes of parameter space yet to explore? Our parameter survey is far from complete. We have chosen a few examples which are interesting and plausibly motivated, but there are many variations possible within similar physical systems. Many physical systems with dust have fast-growing RDIs, but with parameters quite different to those studied here (e.g. AGN and their outflows, dense GMCs, supernova remnants, the ISM of primordial neutral galaxies, planetary atmospheres, protostellar discs; see Paper I for further discussion).

(vii) How does the introduction of additional grain physics modify the predictions here? As noted above, in the non-linear regime, some of our simulations reach dust concentrations $\gg 10^{9}$ times the mean density. Obviously, other physics will become important long before these densities are reached, including e.g. dust self-shielding (if radiation drives the differential acceleration), self-gravity of the grains, grain collisions, and dust current. Some of these could suppress clustering, but others could make it stronger. The high densities and low local relative grain-grain velocities in these regimes may make them ideal for coagulation. But much of the volume being dust-poor might suppress dust growth via accretion of ions from the gas. In the disperse regime, some of our simulations produce isotropic dust velocities many times the sound speed, which may introduce sputtering and shattering in grain collisions.

Finally, we stress that we have explored only the ideal MHD case of the Squire \& Hopkins (2018b) instabilities. A host of other instabilities exist which appear when other physics are present (e.g. external or self-gravity, stratification, centrifugal or Coriolis forces, non-ideal MHD, kinetic MHD, strong coupling of multiply scattered radiation, etc.). In future work, we hope to explore these cases in more detail, together with some of the questions above. 


\section{ACKNOWLEDGEMENTS}

We thank Alexander Kaurov and Ulrich Steinwandel for a number of enlightening discussions and useful comments. Support for PFH was provided by an Alfred P. Sloan Research Fellowship, NSF Collaborative Research Grant \#1715847 and CAREER grant \#1455342, and NASA grants NNX15AT06G, JPL 1589742, $17-$ ATP17-0214. Support for JS was provided by Marsden Fund grant UOO1727 and a Rutherford Discovery Fellowship, managed through the Royal Society Te Aparangi. Numerical calculations were run on the Caltech compute cluster 'Wheeler', allocations from XSEDE TG-AST130039 and PRAC NSF.1713353 supported by the NSF, and NASA HEC SMD-16-7592.

\section{REFERENCES}

Antal T., Droz M., Lipowski A., 2002, Phys. Rev. E, 66, 062301

Apai D. A., Lauretta D. S., 2010, in Apai D., Lauretta D. S., eds, Protoplanetary Dust: Astrophysical and Cosmochemical Perspectives. Cambridge Univ. Press, Cambridge

Aranson I. S., Tsimring L. S., 2006, Rev. Mod. Phys., 78, 641

Ayliffe B. A., Laibe G., Price D. J., Bate M. R., 2012, MNRAS, 423, 1450

Bai X.-N., Stone J. M., 2010a, ApJ, 722, 1437

Bai X.-N., Stone J. M., 2010b, ApJ, 722, L220

Bell A. R., 2004, MNRAS, 353, 550

Ben-Naim E., Krapivsky P. L., 2000, Phys. Rev. E, 61, R5

Bracco A., Chavanis P. H., Provenzale A., Spiegel E. A., 1999, Phys. Fluids, 11,2280

Cadiou C., Dubois Y., Pichon C., 2019, A\&A, 621, A96

Carballido A., Stone J. M., Turner N. J., 2008, MNRAS, 386, 145

Colbrook M. J., Ma X., Hopkins P. F., Squire J., 2017, MNRAS, 467, 2421

Cuzzi J. N., Hogan R. C., Paque J. M., Dobrovolskis A. R., 2001, ApJ, 546, 496

Dorschner J., 2003, in Henning T. K., ed., Lecture Notes in Physics, Vol. 609, Astromineralogy. Springer-Verlag, Berlin, p. 1

Draine B. T., 2003, ARA\&A, 41, 241

Draine B. T., Sutin B., 1987, ApJ, 320, 803

Ernst M. H., Brito R., 2002, J. Stat. Phys., 109, 407

Fessler J. R., Kulick J. D., Eaton J. K., 1994, Phys. Fluids, 6, 3742

Genel S., Vogelsberger M., Nelson D., Sijacki D., Springel V., Hernquist L., 2013, MNRAS, 435, 1426

Gualtieri P., Picano F., Casciola C. M., 2009, J. Fluid Mech., 629, 25
Hopkins P. F., 2013a, MNRAS, 430, 1653

Hopkins P. F., 2013b, MNRAS, 430, 1880

Hopkins P. F., 2014, ApJ, 797, 59

Hopkins P. F., 2015, MNRAS, 450, 53

Hopkins P. F., 2016a, MNRAS, 455, 89

Hopkins P. F., 2016b, MNRAS, 462, 576

Hopkins P. F., 2017, MNRAS, 466, 3387

Hopkins P. F., Lee H., 2016, MNRAS, 456, 4174

Hopkins P. F., Raives M. J., 2016, MNRAS, 455, 51

Hopkins P. F., Squire J., 2018a, MNRAS, 479, 4681 (Paper I)

Hopkins P. F., Squire J., 2018b, MNRAS, 480, 2813

Johansen A., Youdin A., 2007, ApJ, 662, 627

Johansen A., Youdin A., Mac Low M.-M., 2009, ApJ, 704, L75

Kohlstedt K., Snezhko A., Sapozhnikov M. V., Aranson I. S., Olafsen J. S. Ben-Naim E., 2005, Phys. Rev. Lett., 95, 068001

Lebreuilly U., Commerçon B., Laibe G., 2019, A\&A, 626, A96

Lee H., Hopkins P. F., Squire J., 2017, MNRAS, 469, 3532

Monchaux R., Bourgoin M., Cartellier A., 2010, Phys. Fluids, 22, 103304

Moseley E. R., Squire J., Hopkins P. F., 2019, MNRAS, 489, 325

Pan L., Padoan P., Scalo J., Kritsuk A. G., Norman M. L., 2011, ApJ, 740, 6

Price D. J., Federrath C., 2010, MNRAS, 406, 1659

Rouson D. W. I., Eaton J. K., 2001, J. Fluid Mech., 428, 149

Ruiz-Chavarria G., Baudet C., Ciliberto S., 1996, Physica D: Nonlinear Phenom., 99, 369

Seligman D., Hopkins P. F., Squire J., 2019, MNRAS, 485, 3991

Squire J., Hopkins P. F., 2017, MNRAS, 471, 3753

Squire J., Hopkins P. F., 2018a, MNRAS, 477, 5011

Squire J., Hopkins P. F., 2018b, ApJ, 856, L15

Squires K. D., Eaton J. K., 1991, Phys. Fluids A: Fluid Dyn., 3, 1169

Su K.-Y., Hopkins P. F., Hayward C. C., Faucher-Giguère C.-A., Kereš D., Ma X., Robles V. H., 2017, MNRAS, 471, 144

Tielens A. G. G. M., 2005, The Physics and Chemistry of the Interstellar Medium. Cambridge Univ. Press, Cambridge

Tricco T. S., Price D. J., Laibe G., 2017, MNRAS, 471, L52

Vazquez-Semadeni E., 1994, ApJ, 423, 681

Weingartner J. C., Draine B. T., 2001a, ApJS, 134, 263

Weingartner J. C., Draine B. T., 2001b, ApJ, 563, 842

Wentzel D. G., 1968, ApJ, 152, 987

Yakhot V., 1997, Phys. Rev. E, 55, 329

This paper has been typeset from a $\mathrm{T}_{\mathrm{E}} \mathrm{X} / \mathrm{LAT}_{\mathrm{E}} \mathrm{X}$ file prepared by the author. 